Aus der Abteilung Mund-, Kiefer- und Gesichtschirurgie (Prof. Dr. med. Dr. med. dent. H. Schliephake) im Zentrum Zahn-, Mund- und Kieferheilkunde der Medizinischen Fakultät der Universität Göttingen

\title{
Spätresultate nach minimalinvasiver Sinusbodenaugmentation
}

\author{
INAUGURAL - DISSERTATION \\ zur Erlangung des Doktorgrades \\ für Zahnheilkunde \\ der Medizinischen Fakultät \\ der Georg-August-Universität zu Göttingen
}

vorgelegt von

Katja Blendermann

aus

Hamburg

Göttingen 2011 
Dekan:

I. Berichterstatter:

II. Berichterstatter/ in:

III. Berichterstatter/ in:
Prof. Dr. med. C. Frömmel

Prof. Dr. med. Dr. med. dent. W. Engelke

Prof. Dr. med. Hommerich

Prof. Dr. med. Oppermann

Tag der mündlichen Prüfung: $\quad$ 15.11.2011 


\section{Inhaltsverzeichnis}

1

.EINLEITUNG 5

1.1

Einführung in das Thema 5

1.2 Anatomie und Physiologie des Sinus maxillaris 8

1.3 Augmentationsmaterialien 10

1.4 Operationstechniken 12

1.5 Erfolgskriterien von Implantaten 15

1.6 Literaturübersicht: Resultate nach Sinusbodenaugmentation 16

1.7 Ziel der Arbeit 20

2. MATERIAL UND METHODE 21

2.1 Patientengruppe 21

2.2 Operationstechnik der Subantroskopischen Laterobasalen Sinusbodenaugmentation (SALSA)

2.3 Verwendete Implantatsysteme 25

2.4 Röntgenuntersuchung 25

2.5 Auswertung 26

2.6 Zahnregionbezogene Analyse des Knochenlagers 27

2.7 Messfolie 28

2.8 Röntgenbildauswertung 29

2.9 Klinische Befunde 30

2.10 Statistische Auswertung

3. ERGEBNISSE 34

3.1 Überlebenszeit der Implantate 35

3.2 Erfolgsrate der Implantate 38

3.3 Verteilung der Implantate auf die verschiedenen Zahnregionen 39

3.4 Implantatverluste 40

3.5 Präoperative vertikale Knochenhöhen 41

3.6 Postoperative vertikale Augmentathöhen 42

3.7 Periimplantäre Verhältnisse 44

3.8 Periotest 47

3.9 Knochenverlust 48

3.10 Fragebogen 50

3.11 Komplikationen 52

3.12 Falldarstellung 53 
4.

DISKUSSION 56

4.1 .Methodenkritik: Radiologische Evaluation 56

4.2 Implantatüberlebensrate 58

4.3 Einfluss der präoperativen Knochenhöhe auf das Implantatüberleben 59

4.4 Einfluss der Implantatlokalisation auf das Implantatüberleben 61

4.5 Einfluss der Sinusmukoperiostperforation auf das Implantatüberleben 62

4.6 .Einfluss des Augmentats auf das Implantatüberleben 64

4.7 Mundhygiene und periimplantäre Gesundheit 65

4.8 .Implantatstabilität 67

4.9 Marginaler Knochenabbau 68

4.10 Vergleich von einzeitiger und zweizeitiger SALSA 70

4.11 Patientenzufriedenheit 71

5. SCHLUSSFOLGERUNG 73

6. ZUSAMMENFASSUNG $\mathbf{7 4}$

7. ANHANG 76

7.1 Abkürzungsverzeichnis 76

7.2 Verzeichnis der Abbildungen, Tabellen und Formeln 77

7.3 Literaturverzeichnis 78

\section{Einleitung}




\subsection{Einführung in das Thema}

Nach Entfernung eines Zahnes kommt es durch die fehlende kaufunktionelle Krafteinleitung über das Parodontium der Zähne in den knöchernen Alveolarfortsatz zu einer physiologischen Inaktivitätsatrophie. Dieser Funktionsverlust führt zu vertikalem und horizontalem Abbau der ehemals zahntragenden Kieferkammabschnitte (Araujo und Lindhe 2005).

Als Ursachen für diesen Resorptionsprozess kommen funktionelle, entzündliche, anatomische, prothetische sowie systemische Faktoren in Betracht (Atwood 1971, Mercier 1985, Fallschlüssel 1986, Iizuka et al. 1992, Bodner et al. 1993). Nach Jahren resultiert die Atrophie im Oberkiefer auf diese Weise oft in einer dünnen Knochenlamelle des Sinusbodens.

Die Atrophie setzt bereits nach wenigen Monaten ein und es kommt nicht nur zu einer Formveränderung, sondern vor allem zu einem erheblichen Substanzverlust des knöchernen Zahnhalteapparates (Atwood 1971, Atwood und Coy 1971, Tallgren 1972).

Es konnte ein enger Zusammenhang zwischen dem Ausmaß der Kieferkammresorption und der seit der Extraktion vergangenen Zeit ermittelt werden (Tallgren 1972, Humphries et al. 1989, Ulm et al. 1992, Araujo und Lindhe 2005).

Innerhalb des ersten Jahres nach Zahnentfernung ist der irreversible Resorptionsvorgang am stärksten. Für den Oberkiefer konnte ein Höhenverlust von 2 bis 3 mm (Wictorin 1964, Tallgren 1972, Zitzmann 2004), für den Unterkiefer von 4 bis $5 \mathrm{~mm}$ ermittelt werden (Carlsson und Persson 1967, Tallgren 1972, Zitzmann 2004). Ab dem zweiten Jahr schreitet der chronisch-progressive vertikale Knochenabbau um circa 0,5 mm pro Jahr fort (Atwood 1971). Der physiologische krestal-vertikale Knochenverlust am Alveolarfortsatz im Oberkiefer beträgt jährlich durchschnittlich $0,1 \mathrm{~mm}$. Die Resorptionsrate ist individuell sehr unterschiedlich, jedoch ist sie am gravierendsten unmittelbar post extractionem (Watzek und Mailath-Pokorny 2000).

Gravierend ist auch der unterschiedliche Resorptionsvorgang zwischen Maxilla und Mandibula in horizontaler Richtung. Im Oberkiefer erfährt der Alveolarfortsatz den Abbau vor allem an den bukkalen und labialen Flächen, wodurch sich der Kieferkamm im Sinne einer zentripetalen Resorption allmählich nach palatinal verlagert (Balogh et al. 1962). Der Oberkiefer verkleinert sich und kann eine relative Retral- und Palatinalposition aufweisen (Zitzmann 2004).

Im Unterkiefer erfolgt die Resorption des Alveolarfortsatzes überwiegend von lingual. Der Kieferkamm kann dadurch eine Bukkal- und Labialposition aufweisen. Durch die beschriebenen gegensätzlichen Resorptionsvorgänge in Ober- und Unterkiefer entstehen häufig ungünstige intermaxilläre Verhältnisse, die erhebliche Probleme bei der Herstellung von Zahnersatz verursachen können.

Inkongruenzen zwischen Prothese und Prothesenlager können die Folge dieser anatomischen Veränderungen sein. Auf Grund der ausgeprägten Kieferkammatrophie, der verschobenen 
intermaxillären Kieferrelation und der fehlenden Prothesen-Adaptation kann es zu einem schlechten Prothesenhalt kommen (Weingart et al. 1996).

In der Vergangenheit war es oft schwierig, für diese Patienten einen ausreichenden Tragekomfort durch konventionelle herausnehmbare Teil- oder Totalprothesen zu erlangen (Garg 1999). Mit Hilfe von enossalen Implantaten kann dem Wunsch der Rehabilitation teilbezahnter oder zahnloser Patienten, nach einem festen, implantat-getragenen Zahnersatz entsprochen werden (Adell et al. 1990, Neukam und Esser 2000). Um Implantate fest im Oberkiefer zu verankern, ist ein ausreichendes Knochenangebot Voraussetzung. Durch das Inserieren von dentalen Implantaten ist die Knochenresorption vorhersehbarer und gewinnt so einen neuen Stellenwert (Neukam et al. 1989, Adell et al. 1990). Für die geringeren Resorptionsprozesse wird die kaufunktionelle Belastung des Knochens über die Implantate (Neukam et al. 1989) sowie eine fehlende Belastung durch schleimhautgetragene Prothesen (Weingart et al. 1992) angenommen.

Die im Alter zunehmende Pneumatisation des Sinus maxillaris führt, neben der fortschreitenden Atrophie des Alveolarkammes nach Zahnextraktion im Prämolaren - und Molarenbereich des Oberkiefers, zu einer weiteren Einschränkung des vertikalen und horizontalen Knochenvolumens. Die zum Teil extreme Pneumatisation der Kieferhöhle und die daraus folgende Reduktion der Knochenmenge zwischen Sinusboden und Krestum des Alveolarkammes schränkt die Möglichkeiten einer Implantatinsertion ein (Olson et al. 1997).

Lundgren und Mitarbeiter fanden in $43 \%$ ihrer geplanten Implantationsregionen im posterioren Oberkiefer eine Restknochenhöhe von $\leq 4 \mathrm{~mm}$ (Lundgren et al. 1996). Zusätzlich weist der posteriore Oberkiefer im Vergleich zum Unterkiefer eine weniger dichte und damit weichere Knochenqualität auf (Typ III-IV nach Lekholm, D4 nach Misch) (Lekholm und Zarb 1985, Misch 1999). Die meist nur dünne Kortikalis und niedrigere Spongiosadichte erschwert eine auf Dauer sichere Verankerung des Implantates (Reinhardt und Kreusser 2000). Folglich kann es zu einer verminderten Primärstabilität und reduzierten Widerstandsfähigkeit gegenüber mechanischer Belastung kommen (Tatum 1986, Ulm et al. 1999). Bei D4-Knochen ist das Risiko von auftretenden Implantatverlusten nachweisbar höher (Jaffin und Bermann 1991).

Im Oberkiefer gelten Implantationen erst ab einem ortsständigen, vertikalen Knochenangebot von mehr als $10 \mathrm{~mm}$ als prognostisch günstig (Neukam und Schultze-Mosgau 2004).

Ist die vertikale Knochenhöhe durch fortschreitende Knochenresorption oder Pneumatisation des Sinus maxillaris für die Implantation zu gering, können augmentative Maßnahmen erfolgen. Das Verfahren der Sinusbodenaugmentation vergrößert die Knochenhöhe im Bereich des Sinusbodens (Smiler et al. 1992).

Dadurch ist es möglich, Implantate auch in Kieferkamm-Abschnitten zu inserieren, in denen die anatomischen Ausgangssituationen primär zur Implantation nicht ausreichen (Higuchi und Block 1993). Mittlerweile ist die Sinusbodenelevation in Kombination mit dentalen 
Implantaten ein standardisiertes Verfahren in der prothetischen Restauration und ästhetischen Rehabilitation bei schwerwiegender Atrophie der Maxilla.

Bei der Sinusliftoperation (SLO) bleibt die orale Konfiguration des Alveolarkammes erhalten. Die Pneumatisation der Kieferhöhle, welche die Ursache für den Hauptteil des knöchernen Abbaus ist, wird rückgängig gemacht.

Boyne und James (1980) und Tatum (1986) beschrieben erstmals die klassische SinusliftFenstertechnik. Hierbei wird der Boden der Kieferhöhle über ein faziales Knochenfenster in der Kieferhöhle angehoben und mit Spongiosa oder Knochenersatzmaterial aufgefüllt.

Von Summers (1994) wurde die sogenannte Osteotomtechnik beschrieben. Über einen transalveolären Zugang ist ein Verfahren mit herabgesetzter Invasivität möglich. Allerdings ist die zu erreichende Augmentathöhe auf wenige Millimeter begrenzt und deshalb nur bei gering atrophierten Alveolarkämmen geeignet.

Die subantroskopische laterobasale Sinusbodenaugmentation (SALSA) wurde von Engelke und Deckwer (1997) angewendet und beschrieben. Es wurde ein minimalinvasiver chirurgischer Zugang entwickelt, über den der Subantralraum mit Augmentationsmaterial gefüllt und mit Hilfe der Subantroskopie kontrolliert wird. Sofern Primärstabilität erzielt werden kann, können die Implantate inseriert werden.

\subsection{Anatomie und Physiologie des Sinus maxillaris}

Der Sinus maxillaris ist die größte der Nasennebenhöhlen. Er hat eine pyramidenförmige Struktur. Die Basis dieser dreiseitigen Pyramide ist gegen die Nasenhöhle und deren Spitze 
gegen den Processus zygomaticus gerichtet. Die Stärke der knöchernen Wände variiert je nach Größe und Ausdehnung der Kieferhöhle. Der Sinus maxillaris grenzt mit der oberen Wand an die Orbita, die Hinterwand wird vom Tuber maxillae gebildet, während die Vorderwand sich vom Orbitaboden bis zum Alveolarfortsatz des Oberkiefers erstreckt (Rosenbauer et al. 1998). Beim vollständig bezahnten Patienten beträgt die Ausdehnung des Sinus maxillaris in anteriorposteriorer Richtung circa $30 \mathrm{~mm}$, in der Region des ersten Molaren circa $30 \mathrm{~mm}$ bis zu 37,5 $\mathrm{mm}$ und in medial- lateraler Richtung circa 25 mm (Triplett und Schow 1996).

Beim Erwachsenen variiert das Volumen der Kieferhöhle individuell von $4,5 \mathrm{~cm}^{3}$ bis zu 35,2 $\mathrm{cm}^{3}$ und beträgt durchschnittlich $15 \mathrm{~cm}^{3}$ (Ariji et al. 1994). Die Größe des Sinus maxillaris besitzt eine beträchtliche Variationsbreite. $\mathrm{Zu}$ einer fortschreitenden Vergrößerung kommt es zum einen durch Pneumatisation, hauptsächlich aber findet sie mit fortschreitendem Alter und vor allem nach Zahnverlust statt. Die Variation des Sinusvolumens ist nicht nur individuell unterschiedlich, sondern kann auch in beiden Sinus einer Person asymmetrisch sein (Chanavaz 1990).

Normalerweise hat der Sinus maxillaris eine konvexe untere Begrenzung mit dem tiefsten Punkt zwischen dem ersten und dem zweiten Molaren. Zwischen dem Boden des Sinus maxillaris und den Zahnwurzeln befindet sich gewöhnlich eine drei bis vier Millimeter dicke Knochenschicht. Die Stärke dieser ist abhängig von Form und Größe der Kieferhöhle und variiert je nach Länge der Zahnwurzeln (Chanavaz 1990). Die mittlere Höhe des Alveolarfortsatzes, die sich aus der Distanz zwischen dem Krestum des Alveolarkammes und dem Kieferhöhlenboden ermitteln lässt, kann beim Erwachsenen zwischen 9,30 mm und 3,23 mm (Max. 13,80 mm, Min. 0,80 mm) betragen (Cawood und Howell 1991).

Am Boden der Kieferhöhle befinden sich häufig knöcherne Septen, sogenannte UnderwoodSepten, die in ihrer Höhe stark variieren können (Watzek et al. 1999). Ihre Durchschnittshöhe beträgt 7,9 mm und kann bis zu 17,0 mm (Ulm et al. 1995) erreichen. Der Sinus maxillaris wird durch die Septen in zwei oder mehr Kompartimente mit eigener Öffnung unterteilt. Das Vorliegen der Underwood-Septen kann die Mobilisation der Sinusmembran zum Teil erheblich erschweren (Valentin et al. 2000).

Die Gefäßversorgung des Sinusbodens sowie der medialen und lateralen Kieferhöhlenwand erfolgt von den sich dort verzweigenden großen und kleinen palatinalen Gefäßen. Sie treten durch den Canalis incisivus und anastomosieren auf der Unterseite des Gaumens mit der A. palatina major. Die A. palatina major und A. palatina minor entspringen der absteigenden A. palatina, stammend aus der A. sphenopalatini (Chanavaz 1990). Der venöse Abfluss findet über die V. facialis, die V. sphenopalatina, und den Plexus pterygoideus statt.

Die sensible Versorgung der Schleimhaut des Sinus maxillaris findet über den N. ethmoidalis anterior, einen Zweig der drei Endäste des N. ophthalmicus statt. Zusätzlich wird die Schleimhaut durch die Nn. alveolares superiores aus dem N. maxillaris innerviert (Chanavaz 1990). 
Die Auskleidung der Kieferhöhle erfolgt durch die Schneidersche Membran. Diese Schleimhaut ist am Periost der knöchernen Oberfläche befestigt (Smiler et al. 1992) und kann nicht als separate Schicht vom Periost unterschieden werden. In gesundem Zustand variiert die Membranstärke zwischen 0,13 mm und 0,5 mm (Drettner 1980). Die Schneidersche Membran besteht aus viellagigen Schichten Zylinderepithel mit einer oberflächlichen Schicht aus zilienhaltigen und nicht zilienhaltigen Zylinderzellen, Basalzellen, schleimbildenden Becherzellen und einer Basalmembran, die sich an die Lamina propria anschließt (Herberhold 1982).

Die Becherzellen produzieren einen Schutzfilm, der die Membran feucht hält und das zilienhaltige Epithel schützt. Dieser Schutzfilm fördert die mukoziliare Bewegung, die in Richtung des Ostium naturale gerichtet ist. Die Zilienschlagfrequenz der Fimbrien beträgt ungefähr 1000 Schläge pro Minute (Watzek et al. 1999). Diese anatomische Drainage der gesunden Höhle erfolgt über den mittleren Nasengang, das Ostium nasotracheale, welches 25 $35 \mathrm{~mm}$ über dem Sinusboden liegt (Smiler et al. 1992).

Der Sinus maxillaris dient als Luft- und Resonanzraum, reduziert das Gewicht des Schädels und trägt zur Modulation der Sprache bei. Weiterhin dient er der Erwärmung der Luft, bevor diese eingeatmet wird.

\subsection{Augmentationsmaterialien}

Für eine schonende und minimalinvasive Behandlungsweise werden nur die Bereiche der Kieferhöhle eleviert und augmentiert, in denen Implantationen erfolgen sollen. 
Für den Sinuslift können verschiedene Materialien verwendet werden:

- Autogene Transplantate

- Allogene Ersatzmaterialien

- Xenogene Ersatzmaterialien

- Alloplastische Ersatzmaterialien.

Autogene (oder auch autologe) Transplantate stammen von dem Individuum, in das sie auch wieder verpflanzt werden. Autologer Knochen besitzt die Fähigkeit zur Osteogenese, d.h. er bewirkt die Knochenneubildung direkt aus Osteoblasten (Misch und Dietsh 1993). Bei vielen Autoren wird das autogene Transplantat als „Goldstandard“ unter den Augmentationsmaterialien (ATM) angesehen. Besonders zur Rekonstruktion größerer Defekte oder bei ersatzschwachem Lager, wie einer sehr geringen subantralen Restknochenhöhe, wird der Einsatz von autologen Transplantaten als Augmentationsmaterial der Wahl beschrieben (Wheeler et al. 1996, Block und Kent 1997, Garg 1999, Langer B und Langer L 1999).

Die osteogene Potenz des körpereigenen Materials garantiert eine funktionstüchtige Struktur (Wheeler et al. 1996, Kreisler et al. 2006).

Autologe Knochentransplantate zeichnen sich durch ihre biologische Wertigkeit aus, d.h. durch ihre hohe biologische Potenz und ihre biomechanischen adäquaten Eigenschaften sind sie besonders geeignet für Augmentationen, wie den horizontalen und vertikalen Kieferkammaufbau (Block und Kent 1997, Riediger 1989). Autologe Spongiosatransplantate haben aufgrund ihrer porösen Struktur nur eine geringe mechanische Belastbarkeit. Daher werden in Bereichen mit erhöhter mechanischer Belastung meist kortikospongiöse Transplantate verwendet (Wagner und Al Nawas 2004).

Für die Gewinnung des autologen Materials ist eine Entnahmemöglichkeit nötig, wodurch sich die Spendermorbidität und das Risiko für das Auftreten von Komplikationen erhöhen. Die Donorregionen zur Transplantatgewinnung richten sich nach der Defektgröße und den anatomischen Gegebenheiten. Spenderregionen im Mund-, Kiefer- und Gesichtsbereich sind intraoral das Kinn, die retromolare Region, der Bereich der Spina nasalis sowie der Tuberbereich (Woo und Le 2004).

Für die Rekonstruktion kleinerer Defekte werden kortikospongiöse und kortikale autologe Transplantate am häufigsten zur Augmentation genutzt. Diese können intraoperativ durch das Abhobeln von Knochenspänen oder beim Vorbohren gewonnen werden. Die Verwendung von Blocktransplantaten oder partikuliertem Knochen ist abhängig von der Defektgröße und -form (Kreisler et al. 2006).

$\mathrm{Zu}$ den Komplikationen bei Transplantationen von autologem Knochen gehören vor allem chronische Schmerzen (Heary et al. 2002), oberflächliche und tiefe Infektionen, neurologische Schädigungen, Herniationen oder Hämatome (Arrington et al. 1996, Goulet et al. 1997, Heary 
et al. 2002, Niedhart et al. 2003). Ein weiterer Nachteil sind die verlängerten Operations- und Liegezeiten (St John et al. 2003) und die limitierte Verfügbarkeit, z.B. bei Osteoporose oder großen Knochendefekten.

Allogene Transplantate stammen von derselben Spezies, jedoch sind Spender und Empfänger verschiedene Individuen. Zu den allogenen Materialien gehören unbehandelte Gewebe und aus diesen durch Weiterbehandlung gewonnene Ersatzmaterialien.

Über Knochenbanken kann der gespendete allogene Knochen bezogen werden, wobei dieser definierten rechtlichen und labortechnischen Anforderungen unterliegt. Durch verschiedene Bearbeitungsverfahren (z.B. Zerkleinerung, Demineralisation, Deproteinisierung) können unterschiedliche Transplantate aus humanem Knochen hergestellt werden.

Bei xenogenen Transplantaten gehören Spender und Empfänger unterschiedlichen Spezies an. Nach erfolgter Sterilisation und Deproteinisierung dürfen die meist bovinen Knochentransplantate als ATM verwendet werden. Bei unbehandelten Transplantaten kann es zur Übertragung von Infektionskrankheiten und damit $\mathrm{zu}$ ausgeprägten immunologischen Reaktionen kommen. Nach erfolgter Entfernung aller Oberflächenproteine sind sie jedoch gut verträglich und in großer Menge verfügbar.

Allogene und xenogene Materialien besitzen eine hohe knochenreparative Fähigkeit. Im Rahmen der Osteokonduktion findet entlang poröser Leitstrukturen der schleichende Ersatz durch das umliegende Knochengewebe statt (Parikh 2002).

Trotz guter klinischer Ergebnisse ist die Verwendung umstritten (Hönig et al. 1999). Auf Grund des Übertragungsrisikos haben die Entwicklung und der Einsatz alloplastischer Knochenersatzmaterialien in den vergangenen Jahren einen höheren Stellenwert eingenommen. Durch die Anwendung von Knochenersatzmaterialien werden autogene Transplantationen unnötig bzw. die benötigte Menge kann reduziert werden (Lane et al. 1999). Knochenersatzmaterialien können alleine oder in Kombination mit anderen Materialien verwendet werden. In der Literatur wird eine Kombination beider Materialien, autogen und alloplastisch, befürwortet (Watzek et al. 1998, Tadjoedin et al. 2000).

Alloplastische Materialien in Kombination mit autogenem Knochen führen $\mathrm{zu}$ einer Materialvermehrung und schützen vor Resorption des autogenen Knochens. Sie ersetzen strukturelle Elemente und fördern den Knochenheilungsprozess (Schnürer et al. 2003). Ziel ist eine baldige und vollständige Durchbauung des Defektes durch vitalen funktionsstabilen Eigenknochen.

Alle Knochenersatzmaterialien und deren Abbauprodukte sind durch ihre Biokompatibilität gekennzeichnet. Kleinste Unterschiede hinsichtlich chemischer Zusammensetzung und kristalliner Struktur zwischen den verschiedenen alloplastischen Knochenersatzmaterialien 
wirken sich auf die physikalischen Eigenschaften in vivo aus. Am häufigsten werden synthetische Materialien eingesetzt. Sie besitzen einen hohen Reinheitsgrad und klar definierte physio- und kristallchemische Eigenschaften (Horch et al. 2006).

\subsection{Operationstechniken}

Ein Sinuslift ist ein chirurgischer Eingriff, bei dem im Oberkieferseitenzahnbereich ein Knochenaufbau erfolgt. Über einen angelegten Zugang kann anschließend Knochenersatzmaterial und/oder autologer Knochen in die Kieferhöhle eingebracht werden. Durch Einlagerung dieser Materialien in den Sinus maxillaris, wird dieser soweit "angehoben" (Lift), dass Implantate gleichzeitig oder nach Einheilung des Augmentationsmaterials sicher verankert werden können.

Um eine ausreichende Primärstabilität erzielen zu können, sollte die vertikale Knochenhöhe nicht unter $10 \mathrm{~mm}$ betragen (Misch 1987). So muss zur Verbesserung eines ungenügenden Implantatlagers eine solche Knochenhöhe erst geschaffen werden.

\section{Fenstertechnik}

Boyne und James (1980) und Tatum (1986) beschrieben diese seit über 20 Jahren durchgeführte Operationsmethode der Sinuslift-Fenstertechnik (SFT) zuerst. Tatum präsentierte 1976 auf einem Implantat-Kongress in Alabama Patientenfälle, bei denen die Schleimhaut des Kieferhöhlenbodens eleviert wurde. Diese wurde über ein angelegtes Fenster auf der fazialen Fläche der Maxilla vom knöchernen Kieferhöhlenboden und der kaudal-vestibulären sowie der Hinterwand vorsichtig gelöst und auf eine angehobene, „geliftete“ Position gebracht. Der entstandene Hohlraum wurde mit autologem Knochen gefüllt.

Boyne und James beschrieben die Sinusbodenelevation über ein Knochenfenster in der lateralen Sinuswand. Bei diesem Verfahren wird mit Hilfe von 2 Osteotomlinien, die knapp über dem Boden des Sinus maxillaris verlaufen, ein Knochendeckel an der lateralen Kieferhöhlenwand angelegt. Anschließend wird die Schneidersche Membran vorsichtig abgelöst und der Deckel nach kranial geklappt. Eine Perforation der Schneiderschen Membran ist unbedingt zu vermeiden. Nach Hochklappen des Deckels entsteht ein Hohlraum, der mit Augmentationsmaterial aufgefüllt wird.

Vorteilhaft bei diesem Zugang ist die Schonung der knöchernen Integrität im Bereich der späteren Implantathalsebene (Neukam und Schultze-Mosgau 2004).

Ist ein einzeitiges Verfahren vorgesehen, werden vor der eigentlichen Augmentation die 
Implantatkavitäten angelegt. Anschließend wird das Augmentationsmaterial an die mediale Wand des Sinus maxillaris angelagert, danach werden die Implantate inseriert. Nacheinander wird dann anterior, posterior und lateral augmentiert (Kent und Block 1989). Abschließend wird der Mukoperiostlappen reponiert und speicheldicht vernäht.

Die Höhe des Restknochens ist ausschlaggebend, ob ein- oder zweizeitig operiert wird. Wenn bei adäquater Höhe und Qualität des Knochenlagers zum Zeitpunkt der Sinusbodenaugmentation die primärstabile Verankerung dentaler Implantate möglich ist, kann ein simultanes Vorgehen gewählt werden.

Einige Autoren empfehlen die simultane Implantation nur unter der strengen Voraussetzung einer Restknochenhöhe von $\geq 4 \mathrm{~mm}$ (Neukam und Schultze-Mosgau 2004). Im Vergleich der Überlebensraten von Implantaten bei Sinusbodenaugmentation mit simultaner Insertion und einem zweizeitigen Vorgehen mit verzögerter Implantation konnte kein signifikanter Unterschied hinsichtlich des Behandlungserfolges festgestellt werden (Wallace und Froum 2003).

Limitierend für das einzeitige Operationsverfahren ist die erzielbare Primärstabilität (Hürzeler et al. 1996), die wesentlich durch die präoperative Knochenhöhe und Knochenqualität beeinflusst wird. In der internationalen Literatur wird eine erzielbare Primärstabilität von dentalen Implantaten bei einer Restknochenhöhe des Kieferkamms von mindestens 4 - 5 mm im maxillären Seitenzahnbereich beschrieben. Die Primärstabilität sinkt mit Abnahme des vertikalen Knochenangebots. Viele Autoren wählen im Bereich um $4 \mathrm{~mm}$ die zweizeitige Vorgehensweise (Jensen et al. 1998).

In Grenzfällen wird empfohlen, die Entscheidung für ein bestimmtes Verfahren intraoperativ nach exakter Messung des Knochenangebots zu treffen (Jensen et al. 1998). Block und Kent (1993) hingegen wählen erst bei einer radiologisch sichtbaren Knochenhöhe von 1- 2 mm die zweizeitige Operationsmethode.

Bei einem zweizeitigen Verfahren werden simultan keine Implantate inseriert. Der geschaffene subantrale Hohlraum wird lediglich mit dem ausgewählten ATM gefüllt. Anschließend wird der Mukoperiostlappen reponiert und speicheldicht vernäht. Die Implantatinsertion erfolgt nach einer Einheilphase von 3-6 Monaten in einer zweiten Operation (Garg 1999).

Mittlerweile existieren verschiedene Modifikationen dieser Sinuslift-Technik, jedoch sind alle über einen Zugang zum Subantralraum durch ein lateral angelegtes Knochenfenster gekennzeichnet (Woo und Le 2004).

\section{Osteotomtechnik}

Ein Nachteil der Operationstechnik nach Tatum ist, dass für den Operationszugang das Ablösen eines großen Mukoperiostlappens erforderlich ist. Alternativ zur Fenstertechnik kann eine 
Sinusbodenelevation auch mittels der weniger invasiven Osteotomtechnik vorgenommen werden, wie ursprünglich von Summers beschrieben (Summers 1994).

Hierbei erfolgt der Zugang zum Sinus maxillaris ausgehend vom Alveolarkamm durch lokale Frakturierung des kortikalen Sinusbodens mit speziellen Instrumenten. Nach krestaler Inzision und Freilegung des Alveolarkamms wird die Implantatkavität bis nah an den Kieferhöhlenboden vorgebohrt. Der Sinusboden wird leicht eingedrückt und mit Osteotomen aufsteigenden Durchmessers wird dieser mit der Schleimhaut verbundene Knochenbereich mechanisch mit leichten Hammerschlägen angehoben und erweitert. Die Kieferhöhlenschleimhaut wird dabei zeltartig eleviert und es entsteht dadurch ein Hohlraum, welcher anschließend durch das Bohrloch mit dem gewählten Augmentat gefüllt wird.

Diese Technik ersetzt die Präparation eines lateralen Knochenfensters (Summers 1994, Fugazzotto 1999, Brägger et al. 2004). Sowohl eine simultane wie auch eine verzögerte Implantation sind möglich.

Allerdings muss für diese Technik eine bestimmte Dicke des Kieferhöhlenbodens (min. $6 \mathrm{~mm}$ ) gewährleistet sein. Bei geringerer Höhe ist eine Primärstabilität des Implantates unter Umständen nicht erzielbar, während das direkte Verfahren ohne gleichzeitige Implantation bis weit unter 1mm Schichtdicke durchführbar ist (Summers 1998, Zitzmann und Schärer 1998, Woo und Le 2004). Summers setzt eine Restknochenhöhe von mindestens $5 \mathrm{~mm}$ für eine ausreichende Primärstabilität des Implantates voraus (Summers 1996).

Von Vorteil bei der Osteotomtechnik ist die leichtere Durchführbarkeit als bei der Fenstertechnik, weshalb sie auch bevorzugt bei Einzelimplantaten angewendet wird. Weiterhin besteht gegenüber der SFT ein geringeres postoperatives Operationstrauma (Iglhaut 2001, Woo und Le 2004).

Allerdings kommt es ohne endoskopische Kontrolle leichter zum Übersehen einer Perforation der Schneiderschen Membran (Nkenke et al. 2002). Außerdem ist der erzielbare Zugewinn an vertikalem Knochenangebot auf 3 bis $5 \mathrm{~mm}$ begrenzt (McAllister und Haghighat 2007). Damit ist diese Operationstechnik nur bei geringen Atrophien im Oberkiefer anwendbar.

\subsection{Erfolgskriterien von Implantaten}

Es besteht ein enger Zusammenhang zwischen dem Langzeiterfolg der Implantation und dem von Brånemark entwickelten Prinzip der Osseointegration, das einen direkten Kontakt 
zwischen Knochen und Implantatoberfläche fordert. Ziel ist es, eine maximale Knochenquantität und -qualität um ein Implantat herum aufzubauen und zu erhalten. Als Misserfolg muss immer Bindegewebe im Implantatkontakt bewertet werden (Brånemark et al. 1969, Brånemark 1985).

Gründe für einen Misserfolg können nach Bert et al. (2005) prinzipiell entweder eine gescheiterte Osseointegration oder der Verlust einer ursprünglich erfolgreichen Osseointegration sein.

Für die Identifikation eines Implantationserfolges können verschiedene klinische und röntgenologische Kriterien angewendet werden. Allerdings werden für die Beurteilung fast ausschließlich Negativkriterien einbezogen, deren Fehlen oder Ausbleiben für einen Erfolg Bedingung ist und bei deren Auftreten ein Misserfolg anerkannt werden muss.

Ein erfolgreiches Implantat muss sich klinisch fest, entzündungs- und schmerzfrei ohne periimplantäre Osteolyse in situ befinden. Die primäre oder nachträgliche Stilllegung eines Implantates muss ebenfalls als Misserfolg gewertet werden, da dieses Implantat funktionell wertlos ist (Naert et al. 1992, Snauwaert et al. 2000).

Diese Kriterien wurden in der vorliegenden Studie ebenso wie die Erfolgsparameter von Jahn und d'Hoedt (1992) zur Bewertung des Implantaterfolgs zu Grunde gelegt:

Ein erfolgreiches Implantat muss sich in situ befinden. Die parodontale Taschentiefe darf an zwei aufeinander folgenden Untersuchungen nicht mehr als $4 \mathrm{~mm}$ betragen. In der vorliegenden Studie erfolgte die Sondierung der Taschen nur einmal während der Nachuntersuchung, jeweils mesial und distal und der Maximalwert wurde notiert.

Die klinische Implantatbeweglichkeit darf den Wert 1 der Klassifikation der Deutschen Gesellschaft für Parodontologie nicht überschreiten (Periotestwerte zwischen -8 und +9).

Der anguläre Knochenverlust als Mittelwert der mesialen und distalen Messung darf im Verhältnis zur konstruktiv enossalen Implantatlänge 30 \% nicht überschreiten.

Die röntgenologische Auswertung wurde an Orthopantomogrammen (OPG) durchgeführt und alle Messungen mit Hilfe der bekannten Implantatlängen kalibriert.

Mesial und distal des Implantates darf röntgenologisch kein beidseitig durchgehender Spalt vorhanden sein, der eine Breite von 0,5 mm überschreitet.

Die subjektive Patienteneinschätzung bezüglich des Behandlungserfolgs darf nicht schlechter als mit der Schulnote „3 = befriedigend“ ausfallen.

\subsection{Literaturübersicht: Resultate nach Sinusbodenaugmentation}

Für eine umfassende Literaturrecherche wurde in der Datenbank Pubmed mit Hilfe der unten genannten Schlagwörter der gegenwärtige Stand der Wissenschaft erarbeitet. 
Resultate Zeitraum der Suche

\begin{tabular}{llll}
\hline Pubmed & Sinus floor augmentation, sinus floor elevation & 374 & April-August 2010 \\
& Minimally invasive & 21 & \\
Long-term results & 32 & \\
Complications & 72 & \\
Success rate, survival rate & 48
\end{tabular}

Tabelle 1

Die nachfolgende Tabelle gibt einen Überblick über die gelisteten Studien zum Thema „Überlebensrate von Implantaten in augmentierten Kieferhöhlen“ der letzten fünf Jahre (20052010).

\begin{tabular}{|c|c|c|c|c|c|}
\hline Studie & Methode & $\begin{array}{l}\text { Patienten- } \\
\text { zahl }\end{array}$ & $\begin{array}{l}\text { Implantat- } \\
\text { zahl }\end{array}$ & $\begin{array}{l}\text { Beobachtungs- } \\
\text { zeit }\end{array}$ & $\begin{array}{l}\text { Überlebens- } \\
\text { rate }\end{array}$ \\
\hline $\begin{array}{l}\text { Johansson et al. } \\
(2010)\end{array}$ & Laterale Fenstertechnik & 61 & 81 & 12-60 Monate & $98,8 \%$ \\
\hline $\begin{array}{l}\text { Tetsch et al. } \\
(2010)\end{array}$ & $\begin{array}{l}\text { Laterale Fenstertechnik/ } \\
\text { Transalveolärer Zugang }\end{array}$ & 983 & 2190 & 15 Jahre & $97,1 \%$ \\
\hline $\begin{array}{l}\text { Meyer et al. } \\
(2009)\end{array}$ & Laterale Fenstertechnik & 20 & 123 & 4 Jahre & $97,6 \%$ \\
\hline $\begin{array}{l}\text { Pjetursson et al. } \\
(2009)\end{array}$ & Transalveolärer Zugang & 181 & 252 & 3,2 Jahre & $97,4 \%$ \\
\hline $\begin{array}{l}\text { Bornstein et al. } \\
(2008)\end{array}$ & Laterale Fenstertechnik & 56 & 111 & 5 Jahre & $98,0 \%$ \\
\hline $\begin{array}{l}\text { Chiapasco et al. } \\
(2008)\end{array}$ & Laterale Fenstertechnik & 692 & 2037 & 59 Monate & $95,8 \%$ \\
\hline $\begin{array}{l}\text { Kahnberg/Vannas } \\
\text {-Löfqvist (2008) }\end{array}$ & Laterale Fenstertechnik & 36 & 153 & 5 Jahre & $100 \%$ \\
\hline $\begin{array}{l}\text { Mardinger et al. } \\
(2007)\end{array}$ & Laterale Fenstertechnik & 55 & 164 & 3 Jahre & $\begin{array}{l}\text { Erfolgsrate } \\
95,1 \%\end{array}$ \\
\hline $\begin{array}{l}\text { Peleg et al. } \\
(2006)\end{array}$ & Laterale Fenstertechnik & 731 & 2132 & 9 Jahre & $97,9 \%$ \\
\hline $\begin{array}{l}\text { Ferrigno et al. } \\
(2006)\end{array}$ & Transalveolärer Zugang & 323 & 588 & 12 Jahre & $94,8 \%$ \\
\hline $\begin{array}{l}\text { Ewers } \\
(2005)\end{array}$ & Laterale Fenstertechnik & 118 & 614 & 13 Jahre & $95,6 \%$ \\
\hline $\begin{array}{l}\text { Wiltfang et al. } \\
(2005)\end{array}$ & $\begin{array}{l}\text { Laterale Fenstertechnik/ } \\
\text { Onlay Augmentation }\end{array}$ & 100 & 565 & 4,5 Jahre & $\begin{array}{l}\text { Erfolgsrate } \\
93,1 \%\end{array}$ \\
\hline
\end{tabular}

\section{Tabelle 2}

Nachfolgend werden veröffentlichte Studien aufgeführt, welche zur Validierung der klinischen Ergebnisse der vorliegenden Untersuchung herangezogen wurden: 
Johansson et al. (2010) untersuchten 61 Patienten, bei denen ein Sinuslift mittels Fenstertechnik durchgeführt wurde. 81 Straumann Implantate wurden inseriert und autogene Knochenchips wurden als ATM intraoperativ von der lateralen Sinus-Wand gewonnen. Die Restknochenhöhe wurde intraoperativ gemessen und betrug zwischen 3 und $10 \mathrm{~mm}$. Zwischen 12 und 60 Monaten variierte die Beobachtungszeit. Der mittlere apikale Knochenverlust bei der letzen Untersuchung betrug $0,73 \mathrm{~mm}$. Der mittlere marginale Knochenverlust lag mesial bei $0,13 \mathrm{~mm}$ und distal bei 0,12 mm. Die Überlebensrate der Implantate betrug 98,8\%.

Tetsch et al. (2010) untersuchten 983 Patienten, bei denen Sinusliftoperationen durchgeführt und insgesamt 2190 Implantate positioniert wurden. Bei 461 Patienten wurde über einen lateralen Zugang augmentiert und es wurden 1207 Implantate inseriert. Als ATM kamen bovines Knochenmaterial, beta-TCP und in einigen Fällen autogener Knochen alleinig zum Einsatz. Die Osteotomtechnik (transalveolärer Zugang) wurde bei 522 Patienten durchgeführt, 983 Implantate wurden gesetzt und zur Augmentation wurden die intraoperativ gewonnenen Knochenchips verwendet. Eine Ruptur der Schneiderschen Membran konnte bei der Osteotomtechnik in 1,5\% und bei der Fenstertechnik in 13,4\% der Fälle dokumentiert werden. Nach 15 Jahren betrug die Überlebensrate für beide Operationstechniken 97,1\%.

Meyer et al. (2009) untersuchten die Langzeit-Ergebnisse von Sinusliftoperationen mit dem Augmentationsmaterial Cerasorb (beta-TCP). Bei 20 Patienten wurde über einen klassischen Zugang die Kieferhöhle augmentiert und in einem zweizeitigen Verfahren mit 123 Implantaten versehen. Die mittlere Beobachtungszeit für die Implantate betrug 4 Jahre. Die mittlere Knochenerhöhung lag bei 16,9 mm. Es konnte eine Überlebensrate von 97,6\% ermittelt werden. Diese ist vergleichbar mit der Überlebensrate anderer Studien, bei denen autologer Knochen als Augmentationsmaterial eingesetzt wurde. Ebenso ist die Resorptionsrate von Cerasorb ähnlich wie bei autologem Knochen.

Pjetursson et al. (2009) analysierten 252 Implantate, die mittels Osteotomtechnik bei 181 Patienten positioniert wurden. Die präoperative Knochenhöhe betrug durchschnittlich 7,5 mm. Deproteinisiertes bovines Knochenmaterial wurde für die Augmentation verwendet. Die Taschensondierungswerte lagen im Durchschnitt bei 3,44 mm. Die Perforationsrate betrug $10 \%$. Bei 1,7\% der Implantate wurde ein fortgeschrittener Knochenverlust von mehr als $2 \mathrm{~mm}$ ermittelt. Die kumulative Überlebensrate nach einer mittleren Beobachtungszeit von 3,2 Jahren betrug 97,4\%. In Bezug auf die Ausgangssituation, konnte eine Überlebensrate von 91,3\% bei einer Restknochenhöhe unter $4 \mathrm{~mm}, 90 \%$ bei einer Restknochenhöhe zwischen 4 und $5 \mathrm{~mm}$ und $100 \%$ bei mehr als $5 \mathrm{~mm}$ festgestellt werden.

Bornstein et al. (2008) untersuchten 56 Patienten, die in einem zweizeitigen Verfahren mit insgesamt 111 Implantaten behandelt wurden. Die Augmentation erfolgte mittels Fenstertechnik und als ATM wurden autogene Knochenchips in Kombination mit deproteinisiertem, bovinen Knochenmaterial oder beta-TCP eingesetzt. Die mittleren 
Taschensondierungswerte lagen bei 4,14 mm. Die mittleren Werte des Periotests wurden bei -3,0 ermittelt. Der mittlere marginale Knochenverlust nach 5 Jahren lag bei 0,33 mm. Die 5Jahres-Überlebensrate betrug $98 \%$.

Chiapasco et al. (2008) führten bei 692 Patienten Sinusliftoperationen mittels Fenstertechnik durch und inserierten 2037 Implantate. Die mittlere Beobachtungszeit betrug 59 Monate. Zur Augmentation wurde intra- oder extraoral gewonnener autogener Knochen verwendet. Die Überlebensrate betrug 95,8\% und die Erfolgsrate 92,5\%.

Kahnberg und Vannas-Löfqvist (2008) untersuchten in ihrer Studie 36 Patienten, bei denen eine SFT in einem zweizeitigen Verfahren durchgeführt wurde. Die Restknochenhöhe betrug 5 $-6 \mathrm{~mm}$. Es wurden 25 unilaterale und 11 bilaterale Augmentationen vorgenommen. Die Beobachtungszeit lag zwischen 3 bis 5 Jahren. Als ATM wurde autologer Knochen vom Beckenkamm, Kinn oder Kieferwinkel oder Bio-Oss verwendet. Insgesamt wurden 153 Implantate inseriert. Der Knochenabbau nach 5 Jahren betrug durchschnittlich 1,6 mm. Die Überlebensrate betrug $100 \%$.

Mardinger et al. (2007) verglichen in ihrer Studie Ergebnisse und Komplikationen der SLO bei Patienten mit einer Ausgangsknochenhöhe zwischen 1 und $3 \mathrm{~mm}$ (study group) mit denen von Patienten mit einer präoperativen Knochenhöhe von mehr als $4 \mathrm{~mm}$ (control group). Die mittlere Beobachtungszeit lag bei 3 Jahren. Die Patienten wurden einzeitig mittels Fenstertechnik operiert und mit bovinem, xenogenen Knochenmaterial augmentiert. Insgesamt betrug die Erfolgsrate 95,1\%. Die Study group bestand aus 25 Patienten mit einer mittleren Restknochenhöhe von 2,2 mm. Es wurden 88 Implantationen vorgenommen. Perforationen der Schneiderschen Membran erfolgten bei 2 Patienten (7\%). Die Erfolgsrate der Implantate betrug 92\%. Die Kontrollgruppe bestand aus 30 Patienten, bei denen 76 Implantate positioniert wurden. Die Perforationsrate wurde bei 10\% ermittelt und die Erfolgsrate der Kontrollgruppe betrug $98,7 \%$.

Peleg et al. (2006) wollten die Langzeitüberlebensrate von einzeitig gesetzten Implantaten nach Sinusbodenaugmentation bei einer präoperativen Knochenhöhe unter $5 \mathrm{~mm}$ ermitteln. Es konnten 2132 Implantate bei 731 Patienten über einen Zeitraum von 9 Jahren beobachtet werden. $20,4 \%$ der Implantate wurden in 1-2 mm Ausgangsknochenhöhe positioniert. Perforationen der Schneiderschen Membran wurden in 16 Fällen festgestellt (2,7\%). Die mittlere Knochenerhöhung betrug 8,4 mm (6,7-9,4 mm). Die kumulative Überlebensrate betrug 97,9\%. Für Implantate in einer Restknochenhöhe von 1-2 mm betrug sie 94,6\% und 98,9\% für Implantate, die in mehr als $5 \mathrm{~mm}$ Knochen platziert wurden.

Ferrigno et al. (2006) operierten 323 Patienten mittels einzeitiger Osteotomtechnik und inserierten 588 Implantate. Die präoperative Knochenhöhe variierte zwischen 6 und $9 \mathrm{~mm}$. Die Augmentathöhe lag bei 4-6 mm. Es konnten 13 Perforationen der Schneiderschen Membran dokumentiert werden (2,2\%). Die mittlere Beobachtungszeit betrug 59 Monate. Der 
durchschnittliche Wert der Sondierungstiefen lag bei 3,4 mm. Für die Augmentation wurden intraoperativ gewonnene autologe Knochenchips verwendet. Die 12-Jahre-Überlebensrate der Implantate betrug $94,8 \%$ und die Erfolgsrate betrug 90,8\%.

Ewers (2005) untersuchte 614 Implantate bei 118 Patienten, die nach der von Tatum beschriebenen Fenstertechnik in einem zweizeitigen Operationsverfahren inseriert wurden. Perforationen der Schneiderschen Membran wurden in 20,6\% beschrieben. Als ATM wurde AlgiPore, welches aus Algen hergestellt und sich in Hydroxylapatit umwandelt, in Verbindung mit PRP (platelet-rich Plasma) und intraoperativ gewonnenem, autogenen Knochen eingesetzt. Die mittlere Restknochenhöhe lag bei 3,6 mm (1-5 mm). Die Volumenresorptionsrate nach 6,4 Monaten betrug $14 \%$ im Vergleich zu 49,5\% nach 6 Monaten bei autogenem Knochen. Die Überlebensrate nach 13 Jahren betrug 95,6\%.

In der Studie von Wiltfang et al. (2005) wurde das Verfahren der Onlay Augmentation mit der klassischen SFT in Bezug auf die Erfolgsrate nach 5 Jahren verglichen. Insgesamt wurden 100 Patienten mit einer präoperativen Knochenhöhe unter $4 \mathrm{~mm}$ in die Studie integriert. Bei 39 Patienten wurden Onlay Augmentationen vorgenommen und 235 Implantate inseriert. Das Sinusliftverfahren wurde bei 61 Patienten angewendet, bei denen 349 Implantationen erfolgten. Bei beiden Operationstechniken wurde Kortiko-Spongiosa vom Beckenkamm transplantiert. Die Erfolgsrate nach 5 Jahren betrug insgesamt 93,1\%. Für das Verfahren der Onlay Augmentation konnte eine Erfolgsrate von 91,5\% und für die Sinusliftoperation 94,6\% ermittelt werden.

Zusammenfassend kann festgestellt werden, dass die Überlebensrate bei Sinusliftoperationen im Mittel bei 97,0 \% liegt (Min. 93,1 \%, Max. $100 \%$ ).

\subsection{Ziel der Arbeit}

Anknüpfend an die Studie von Engelke et al. (2003) zur Anwendung der minimalinvasiven Sinusliftoperation (SALSA) sollen in dieser Studie die Langzeitergebnisse evaluiert werden.

Ziel der vorliegenden Studie ist es $\mathrm{zu}$ ermitteln, welchen Einfluss Implantattyp, 
Augmentationsmaterial und Ausgangsknochenhöhe auf die Langzeitergebnisse haben.

Des Weiteren soll radiologisch der Knochenabbau des Implantatlagers sowie der periimplantäre Zustand der Implantate erhoben werden. Außerdem wird durch eine Befragung der Patienten ihre Zufriedenheit mit der Behandlung untersucht. Komplikationen, die während oder nach der Behandlung entstanden, sollen dargestellt werden.

\section{Material und Methode}




\subsection{Patientengruppe}

Die untersuchte Gruppe umfasst Patienten der Mund-, Kiefer- und Gesichtschirurgie des Universitätsklinikums Göttingen, die aufgrund der unzureichenden präoperativen Knochenhöhe vor Implantation mithilfe der minimalinvasiven Sinusliftoperationstechnik (SALSA) behandelt wurden. In dem Untersuchungszeitraum von 1996-2007 wurde bei 94 Patienten, davon 37 Männer und 57 Frauen, eine Sinusbodenaugmentation durchgeführt. Das Durchschnittsalter betrug 63,6 Jahre und die Altersspanne reichte von 25 bis 76 Jahre. Der weibliche Durchschnitt lag bei 63,8 Jahren, der männliche Durchschnitt bei 63,3 Jahren. Der mittlere Beobachtungszeitraum betrug 78 Monate (Min. 3 Monate, Max. 166 Monate).

\subsection{Operationstechnik der Subantroskopischen Laterobasalen Sinusboden- augmentation (SALSA)}

Von Engelke et al. (2003) wurde ein minimalinvasiver Zugang zum Subantralraum (SAR) beschrieben, der durch eine knöcherne Trepanationsöffnung von $5 \mathrm{~mm}$ Durchmesser gekennzeichnet ist. Über die mikrochirurgische Tunnellierungstechnik wird unter videoendoskopischer Kontrolle das Sinusmukoperiost (SMP) vom Boden der Kieferhöhle vorsichtig gelöst und der Subantralraum vergrößert. Im weiteren Verlauf erfolgt die Präparation der Implantatkavitäten und die Kieferhöhle wird schrittweise mit Augmentationsmaterial gefüllt. Sofern Primärstabilität erzielbar ist, können die Implantate positioniert werden.

\section{Operativer Zugang}

Unter Lokalanästhesie wird abhängig von der Anzahl und Lage der geplanten Implantatkavitäten vom Prämolarenbereich bis zum Tuber krestal inzidiert mit mesialen Entlastungschnitten ins Vestibulum. Dann erfolgt die Bildung des Mukoperiostlappens, so dass das inferiore Drittel des Ansatzes der Crista zygomatico-alveolaris sichtbar und der basale Abschnitt der Fossa canina zugänglich ist. Die Trepanationsöffnung zum SAR wird mit einer großflächigen diamantierten Kugelfräse (3-5 mm) an der laterobasalen Wand des Recessus alveolaris der Kieferhöhle mesial der Crista zygomatico-alveolaris angelegt. Die Position des Zugangs sollte immer an der inferiorsten Stelle des Recessus alveolaris angelegt werden, um die laterobasale Tunnellierung zu erleichtern.

\section{Mikrochirurgische Präparation des Subantralraumes}


Die Trepanation des Knochens wird bis unmittelbar an die Schneidersche Membran durchgeführt. Eine Perforation dieser ist unbedingt zu vermeiden. Die Schleimhaut wird zirkulär vom Boden der Kieferhöhle abgelöst und der Zugang wird anschließend auf eine $5 \mathrm{~mm}$ runde Kavität vergrößert.

Unter stützvideoendoskopischer Kontrolle ( $30^{\circ}$ Stützendoskop n. Engelke mit den HopkinsOptiken $30^{\circ}$ und $70^{\circ}$, Storz 7208 BA/CA und Optikschaft 655001) wird das SMP mit speziellen Raspatorien und Elevatoren unter Knochenkontakt angehoben, bis der geschaffene Raum einer ausreichenden Größe für die Aufnahme des ATM entspricht.

Bis $5 \mathrm{~mm}$ distal der letzten zu setzenden Implantatkavität wird immer getunnelt, so dass der gesamte SAR augmentiert werden kann. Die Präparation des SAR wird mittels Subantroskopie kontrolliert. Mit Hilfe des Endoskops können unbeabsichtigt entstandene Perforationen diagnostiziert und mit einer resorbierbaren Folie (Ethicon, Norderstedt) abgedeckt werden.

Zur Stabilisierung eines sehr dünnen SMP können resorbierbare Membranen auch aus prophylaktischen Gründen verwendet werden.

\section{Präparation des Implantatlagers}

Je nach verwendetem Implantatsystem werden die Implantatkavitäten entsprechend der Herstellerangaben präpariert. Auch hier ist ein besonderes Augenmerk auf den Schutz des SMP zu legen und dieses mit geeigneten Instrumenten vor Perforationen zu schützen. Um eine adäquate Befüllung des SAR und Umschichtung der Implantate mit Augmentationsmaterial in allen Bereichen zu gewährleisten, sollten zwischen Implantat und Grenzen des SAR ca. $5 \mathrm{~mm}$ liegen. Diese Grenzbereiche können bei Sondierung der Implantatkavitäten subantroskopisch überprüft werden.

\section{Einzeitige Operationsmethode}

Das Befüllen des SAR mit fraktioniertem ATM beginnt im lateralen Bereich bis zur distalen Implantatkavität (siehe Abb. 1). Als ATM kommen Patientenblut, autologer Knochen und alloplastische Knochenersatzmaterialien (AlgiPore, Bioresorb, Cerasorb) zum Einsatz. Dann wird das distale Implantat in die präparierte Kavität inseriert. Anschließend erfolgt die Umschichtung des distalen Implantates mit Augmentat. Mit einem Kugelstopfer wird das Augmentationsmaterial ohne Druckanwendung leicht kondensiert. Unter videoendoskopischer Kontrolle wird die erfolgte Augmentation und Implantation sowie das gewünschte Aufspannen des SMP begutachtet. 
Je nach Anzahl der Implantate wird schrittweise in mesialer Richtung vorgegangen. Bevor das am weitesten mesiale Implantat positioniert wird, findet die Befüllung des mesialen Abschnitts des SAR statt.

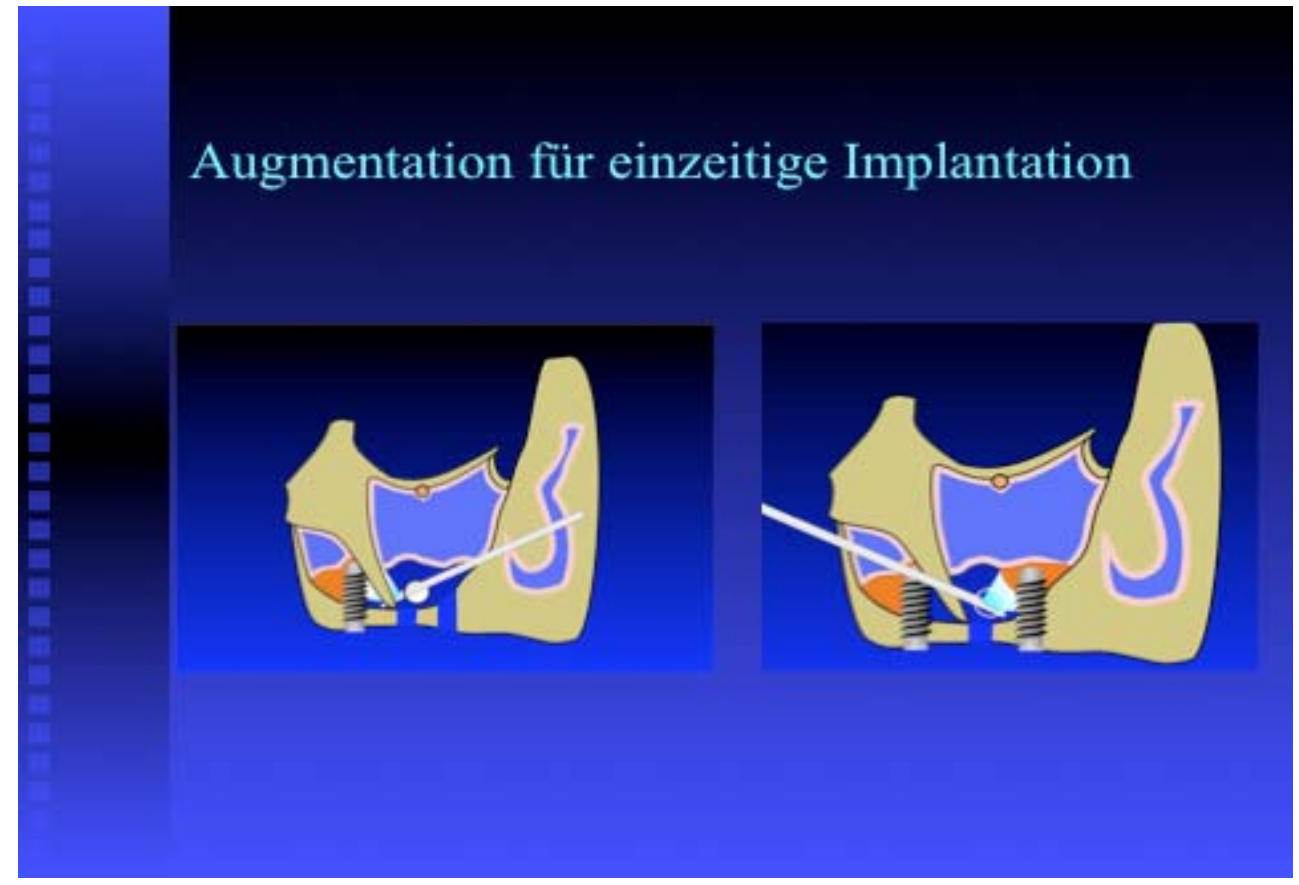

\section{Abbildung 1}

\section{Zweizeitige Operationsmethode}

Ist keine Primärstabilität der Implantate zu erzielen, können die Implantate nicht simultan mit der Augmentation inseriert werden. Es erfolgt die Befüllung des SAR mit fraktioniertem Augmentat distal beginnend und wird anschließend im mesialen und zuletzt im medianen Bereich des SAR fortgeführt. Die Implantatinsertion bei der zweizeitigen SALSA erfolgt 6- 12 Monate postoperativ (siehe Abb. 2). 


\section{Augmentation für zweizeitige Implantation}

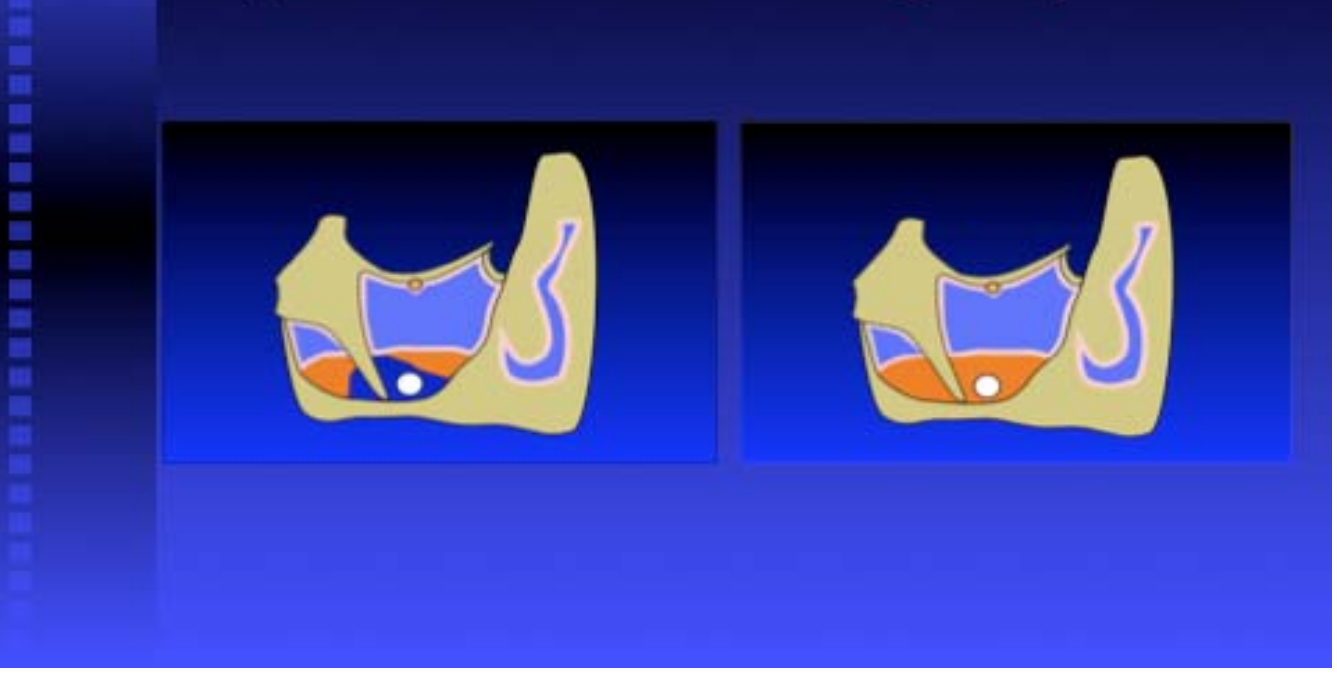

\section{Abbildung 2}

\section{Wundverschluss}

Die Trepanationsöffnung wird verschlossen, in dem der Mukoperiostlappen reponiert und speicheldicht vernäht wird. Der zusätzliche Verschluss des Zugangs mit einer Membran kann in einigen Fällen geeignet sein. Besteht der Verdacht, dass das Augmentat disloziert wurde oder ist eine endoskopische Untersuchung erforderlich, so kann eine sinuskopische Kontrolle mittels Kieferhöhlenpunktion unter Sicht indiziert sein. Meistens ist die Sinuskopie jedoch verzichtbar.

\section{Postoperative Behandlung}

Peri- und postoperativ erhielten die Patienten standardmäßig bis zum dritten postoperativen Tag einschließlich 3 x 1 g Amoxicillin und Paracetamol (4 x 500 mg). Nach 10 Tagen wurden die Nähte entfernt. Soweit keine Interferenzen mit den gesetzten Implantaten vorlagen, durften die Patienten ihre Prothesen tragen. 


\section{Video-Endoskopie}

Endoskopische Kontrollen erfolgten mittels $30^{\circ}$ - und $70^{\circ}$ - Storz 2,7mm- Hopkins-Optik, die mit einem Storz 487 B Endoskop (Storz, Tuttlingen) verbunden wurden. Die Lichtquelle bestand aus einer 300-W 6,000 K Xenon Kaltlichtfontäne (Storz). Die Aufzeichnung konnte mit einem VHS Videorecorder (Sony, Köln) durchgeführt werden.

\subsection{Verwendete Implantatsysteme}

In der Studie wurden über den Beobachtungszeitraum von über 13 Jahren vier Implantatsysteme verwendet. Insgesamt wurden 320 Schraubenimplantate verschiedener Hersteller inseriert:

- 116 Semados Implantate (Fa. Bego, Bremen)

- 118 Xive Implantate (Fa. Friadent, Mannheim)

- 50 ITI Implantate (Fa. Straumann, Freiburg)

- 36 Frialit-2 Implantate (Fa. Friadent, Mannheim).

\subsection{Röntgenuntersuchung}

Für die Auswertung wurden die prä-, postoperative und die aktuellste OPG-Aufnahme verwendet. Die Aufnahmen erfolgten mit folgenden Geräten:

Philips Orthoralix 9200 bei 65-74 KV und 6-8 mA mit einer Aufnahmedauer von 12s sowie Sirona Orthophos XG Plus DS/Ceph bei 69- $75 \mathrm{KV}$ und $8 \mathrm{~mA}$ mit einer Aufnahmedauer von $14 \mathrm{~s}$.

Für den Philips Orthoralix 9200 wurden Kodak T- Mat G/RA Dental Filme mit dem Format 15 x 30 und für den Sirona Orthophos Kodak T- Mat G/RA Dental Filme mit dem Format 20 x 25 verwendet. Die Röntgenkassetten sind mit hochqualitativen Seltene-Erden-Verstärkerfolien augestattet (Kodak-Lanex-Regular-Folie). Die Entwicklung der Filme erfolgte mit dem Entwickler 3 M XP 505.

Der durchschnittliche Vergrößerungsfaktor beider verwendeter Orthopantomographen beträgt 1,25. Er wurde bei vorherigen Vergleichsmessungen ermittelt. 


\subsection{Auswertung}

Erhobene Daten:

- Die persönlichen Daten der Patienten :

- Geburtsdatum

- Geschlecht

- Die operationsbedingten Daten der Patienten :

- Lokalisation der Implantationsstelle (Zahnregion)

- Präoperative Knochenhöhe

- Augmentationsmaterial

- Implantatsysteme

- Implantatlänge und - durchmesser

- Perforationen der Schneiderschen Membran

- Verwendung einer Vicrylmembran

- intraoperative Komplikationen

- Einzeitiges oder zweizeitiges Vorgehen

- Postoperative Komplikationen

- Postoperative Augmentathöhe (ATH)

- marginaler Knochenabbau

- apikaler Knochenabbau

- Periotest-Werte

- Sondierungstiefen

- Sulcus-Blutungs-Index

- Prothetische Konstruktion 


\subsection{Zahnregionbezogene Analyse des Knochenlagers}

Eine Beurteilung des Implantatlagers sowie der angrenzenden anatomischen Strukturen ist bei teilbezahnten Patienten, besonders wenn es sich um zu versorgende Schaltlücken handelt, weitaus einfacher durchzuführen, als im zahnlosen posterioren Oberkiefer. Aus diesem Grund entwickelten Engelke et al. (2003) ein Messschema, das eine genaue Analyse des Knochenlagers nach ehemaligen Zahnregionen im OPG erlaubt.

Anatomische, auf jedem OPG leicht zu erkennende Strukturen wurden hierfür einbezogen. Die Strecke einer horizontal zum Nasenboden verlaufenden Linie zwischen der Mitte des mesialen Mittelgesichtspfeilers bis zur Hinterwand der Kieferhöhle entspricht der Region von Mitte des Caninus bis zur distalen Wand des dritten Molaren. Zusammen mit den Werten von Durchschnittsbreiten der Oberkieferzähne (Berkovitz et al. 1992) und der gemessenen Strecke wurde eine Formel aufgestellt, mit derer die orthopantomogram-bezogenen Zahnbreiten ermitteln werden können.

Folgende Durchschnittsbreiten wurden ermittelt:

\begin{tabular}{|c|c|c|c|c|c|c|}
\hline Oberkieferzahn & C & PM1 & PM2 & M1 & M2 & M3 \\
\hline $\mathrm{mm}$ & 7,5 & 7 & 7 & 10,5 & 9,5 & 8,5 \\
\hline
\end{tabular}

Tabelle 3

(Berkovitz et al. 1992)

Durch Addition der mm-Angaben, wobei beim Caninus nur die halbe Zahnbreite (also 3,725 $\mathrm{mm}$ ) einbezogen wird, und anschließendes Teilen des Betrags durch die jeweiligen Durchschnittszahnbreiten, resultiert ein Zahnregion-bezogener Faktor (a).

\begin{tabular}{|l|c|c|c|c|c|c|}
\hline Zahnbreite & $1 / 2 \mathrm{C}$ & PM1 & PM2 & M1 & M2 & M3 \\
\hline Faktor $\mathrm{a}_{(3-8)}$ & 0,09 & 0,15 & 0,15 & 0,23 & 0,20 & 0,18 \\
\hline
\end{tabular}

Tabelle 4 
Mit Hilfe der ermittelten Formel lässt sich, vom Vergrößerungsfaktor unabhängig, die jeweilige Zahnbreite für das betreffende OPG errechnen.

\section{Formel 1}

$y=a_{(3-8)} \times x$

$\mathrm{y}=$ die auf das jeweilige OPG bezogene zu ermittelnde Zahnbreite

$a_{(3-8)}=$ Zahnregion-bezogener Faktor, der sich aus den Durchschnittszahnbreiten ergibt

$\mathrm{x}=$ gemessene Strecke zwischen der Mitte des mesialen Mittelgesichtspfeilers und der Hinterwand der Kieferhöhle des jeweiligen OPGs

\subsection{Messfolie}

Engelke et al. (2003) entwickelten für standardisierte Messungen am OPG eine durchsichtige Messfolie (siehe Abb. 3, S.29), die mit den Ergebnissen der Formel $\mathrm{y}=\mathrm{a}_{(3-8)} \times \mathrm{x}$ erstellt wurde. Die horizontalen Linien enthalten die Werte für $\mathrm{x}$, wobei für die oberste Linie empirisch $35 \mathrm{~mm}$ und für die unterste Linie $60 \mathrm{~mm}$ festgelegt wurden.

Die vertikalen Linien, die in einem von links nach rechts vergrößernden Winkel gezeichnet sind, stellen die y-Werte (jeweilige Zahnbreite) dar. Die gepunkteten vertikal verlaufenden Linien, die in einem sich von links nach rechts vergrößernden Winkel gezeichnet sind, geben die Positionen der jeweiligen Zahnbreitenmitten wieder. Die vertikalen, parallel verlaufenden, abwechselnd gepunkteten oder durchgehend dünn gezeichneten Linien sollen das Anlegen an die Hinterwand der Kieferhöhle und damit die Ausrichtung der Folie auf dem OPG erleichtern. Die durchgehend dick gezeichnete waagerechte Linie ermöglicht ein besseres Anlegen der Folie in der Horizontalen auf dem OPG (parallel zum Nasenboden). 


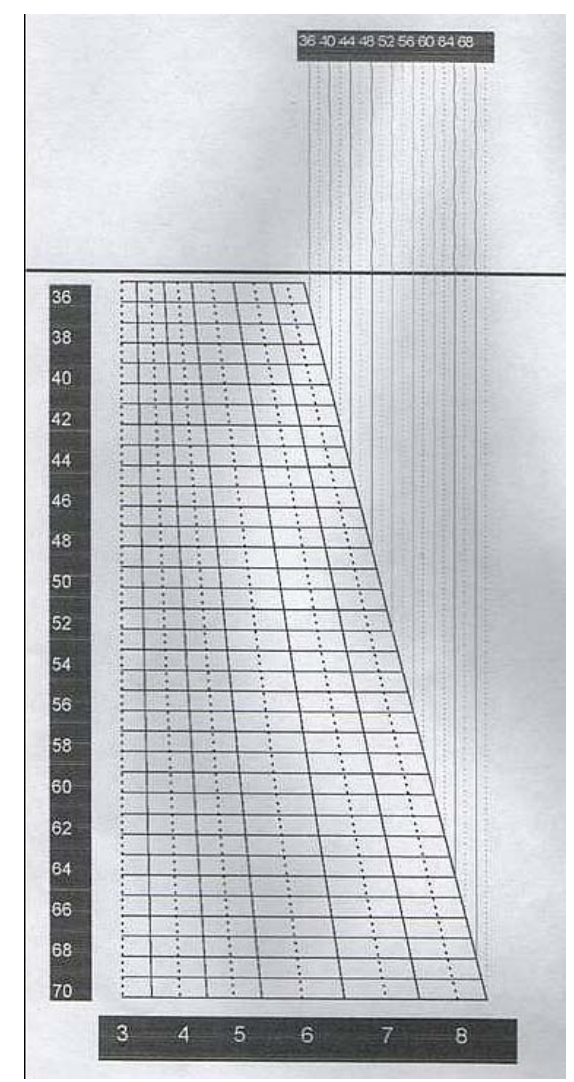

Abbildung 3

\subsection{Röntgenbildauswertung}

Die Auswertung sämtlicher Aufnahmen erfolgte anhand eines Röntgenbild-Betrachters "'MAIER DSK 15x30" (Fa. Maier, Deutschland) und einer Röntgenbildlupe mit 2,5 facher Vergrößerung (X-Produkter, Schweden). Die Vermessung der OPGs wurde von einer Person mit Hilfe eines Geometriedreiecks (Herlitz, Berlin) mit einer 0,5 mm Ablesegenauigkeit durchgeführt.

Der radiologischen Auswertung liegt die vergleichende Inspektion der Röntgenbilder zugrunde, wobei das Implantat als Bezugsgröße diente. Als konstanter Bezugspunkt wurde die Implantatschulter ausgewählt und die Distanzen zwischen ihr und den veränderlichen knöchernen Punkten gemessen.

Der horizontale Knochendefekt wurde mesial und distal des Implantates ermittelt und der Maximalwert pro Implantat wurde für die Auswertung berücksichtigt. Des Weiteren wurde der apikale Knochenabbau am Implantatapex gemessen. Ein wesentlicher Faktor der Orthopantomographie besteht bei der Vergrößerung. Der Vergrößerungsfaktor der Aufnahmen von 1, 25 wurde berücksichtigt und ausgeglichen. 


\subsection{Klinische Befunde}

Im Rahmen der Befunderhebung wurden der Gesamtzustand des Gebisses, die Sondierungstiefen, Bluten auf Sondieren, der Sulcus-Blutungs-Index (SBI), die Werte des Periotests sowie die gewählte Suprakonstruktion erhoben. Für die metrischen Bestimmungen wurde eine kalibrierte Parodontalsonde (PCP 11, Fa. Aesculap, Tuttlingen) herangezogen.

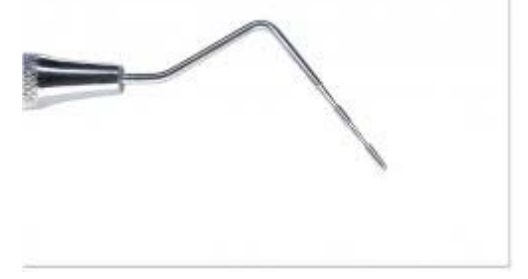

Abbildung 4

\section{Taschensondierungstiefe und Sondierungsbluten (Bleeding on Probing, BOP)}

Um den Zustand der periimplantären Gingiva zu erfassen, wurde die Sondierungstiefe und Sondierungsblutung ermittelt. Die Höhe der Sulcustiefe am Implantat konnte durch die 2Punkte-Messung mesial und distal mit einer kalibrierten Parodontalsonde bestimmt werden. Die Sonde wurde dabei parallel zur Implantatachse unter einem geringen Kraftaufwand von ca. 0,2 N in den Sulkus eingeführt, bis ein leichter Widerstand spürbar war. Die klinische Distanz zwischen Margo gingivae und Taschenfundus parallel zur Implantatachse wurde bestimmt.

Für die Auswertung wurde der mesial und distal am Implantat gemessene Maximalwert in mm herangezogen. Ebenfalls wurde erfasst, ob bei den Messpunkten die periimplantäre Schleimhaut eine Blutung beim Sondieren aufwies. Das Ergebnis der Sondierungsblutung wurde als positiv oder negativ in das Untersuchungsprotokoll eingetragen.

\section{Modifizierter Sulcus-Blutungs-Index}

Zur Beurteilung des Entzündungsgrades der Gingiva wurde der SBI mit einer Parodontalsonde durchgeführt. Es wurden Blutungen im Bereich der Interdentalräume in Form einer Ja/NeinEntscheidung bewertet. Die Erhebung erfolgt im ersten und dritten Quadranten vestibulär und im zweiten und vierten Quadranten oral. Die Ergebnisse werden in Prozent angegeben. 


\section{Mobilität}

Die Osseointegration der Implantate wurde mit Hilfe des Periotestverfahrens beurteilt. Dieses wurde entwickelt um den Funktionszustand des Parodontiums objektiv und reproduzierbar beurteilen zu können (Schulte et al. 1983). Das Periotestgerät (Periotest S, Medizintechnik Gulden, Modautal) misst dynamisch die Reaktion des Parodontiums auf eine definierte Stoßbelastung.

Bei diesem maschinellen Messverfahren wird ein elektronisch gesteuerter Stößel beschleunigt und in dem Moment, wo er auf das Implantat trifft, abgebremst. Die Abbremsung erfolgt umso schneller, je größer die Dämpfung des Implantats ausfällt, d.h. je besser die knöcherne Integration ist. Aus der Verzögerung wird mit Hilfe eines Mikroprozessors der Periotestwert zwischen -08 und +50 berechnet.

Eine wichtige Rolle bei der Ermittlung aussagekräftiger und reproduzierbarer Werte spielt die korrekte rechtwinklige Haltung des Periotest-Handstückes zum Implantatpfeiler. Auch der Messort, also die Stelle, auf die der Stößel perkutiert, muss bei allen Messungen gleich sein (d'Hoedt et al. 1985). Mit Hilfe des Periotests ergibt sich eine relativ zuverlässige metrische Erfassung der knöchernen Integration durch Messung des Dämpfungsverhaltens der Pfeiler (Schulte et al. 1983).

Der Periotestwert korreliert eng mit der Zahnbeweglichkeit, kann jedoch nicht als direkte Beweglichkeitsmessung angesehen werden. d'Hoedt et al. (1985) stellten einen Zusammenhang zwischen Periotestwert und klinischer Zahnbeweglichkeit her, der in der Tabelle 5 dargestellt ist.

\section{Lockerungsgrad}

0 klinisch fest

I fühlbar beweglich

II sichtbar beweglich

III beweglich auf Lippen und Zungendruck

\section{Periotestwert}

-08 bis +09

+10 bis +19

+20 bis +29

+30 bis +50

Tabelle 5 : Beziehung zwischen Periotest und klinischem Lockerungsgrad 


\section{Patientenzufriedenheit}

Mit Hilfe eines selbst entwickelten Fragebogens konnte nach der subjektiven Einschätzung durch den Patienten eine Beurteilung des Sinusliftverfahrens anhand der Schulnotenscala durchgeführt werden.

Folgende Fragen wurden zur Erfassung der Zufriedenheit von den Patienten subjektiv beantwortet und nach dem deutschen Schulnotensystem bewertet $(1=$ sehr gut, $2=$ gut, $3=$ befriedigend, $4=$ ausreichend und $5=$ mangelhaft):

1. Frage zur Zufriedenheit des Gesamtresultates: „Wie schätzen Sie das Gesamtresultat der Behandlung ein?"

2. Frage zum Halt der Prothese: „Wie schätzen Sie den Halt Ihrer Prothese ein?“

3. Frage zur Kaufunktion: „Wie schätzen Sie Ihre Kaufunktion insgesamt ein?“

4. Frage zur Häufigkeit von Zahnarztbesuchen: „Wie oft mussten Sie aufgrund von Problemen oder Beschwerden mit ihrem Zahnersatz einen Zahnarzt aufsuchen?“

\section{Suprakonstruktion}

Abhängig von der jeweiligen Implantatindikation und den Patientenwünschen wurde entweder festsitzender Zahnersatz in Form von Einzelkronen und Brücken oder herausnehmbarer Zahnersatz in Form von teleskop- und steggestützten Teilprothesen und teleskopierenden Totalprothesen angefertigt. 


\subsection{Statistische Auswertung}

Die Beschreibung der ermittelten Daten erfolgt durch die Angabe von Median, Minimum- und Maximumwert sowie des Mittelwerts. Als Dispersionsmaß fanden entsprechend die Standardabweichung und der Interquartilsabstand, die Differenz zwischen 75\%- und 25\%Quartil, Anwendung.

Bei intervallskalierten Merkmalen geschah die Analyse eines signifikanten Unterschiedes durch die Ermittlung von Kontingenzintervallen mit einem Konfidenzniveau von 95\%. Das Signifikanzniveau wurde für alle Testverfahren mit $\mathrm{p} \leq 0,05$ festgelegt. Zur Darstellung der ermittelten Daten und Ergebnisse wurden Tabellen, Histogramme, Kreisdiagramme und Kurvendiagramme gewählt.

Der Implantationserfolg wurde im zeitlichen Verlauf mit Hilfe der Kaplan-MeierÜberlebensstatistik dargestellt. Dabei markieren die Stufen im Verlauf der Kaplan-MeierKurven Zeitpunkte, an denen Misserfolge eintraten. 


\section{Ergebnisse}

Im Rahmen dieser retrospektiven Untersuchung zur SALSA-Operationsmethode wurden bei 94 Patienten 133 Augmentationen (67 rechts und 66 links) durchgeführt. Von diesen waren 55 Augmentationen unilateral und 39 bilateral erfolgt. Es wurden insgesamt 320 Implantate inseriert. Die einzeitige operative Vorgehensweise wurde bei 122 Kieferhöhlen mit insgesamt 295 Implantaten angewendet. Die zweizeitige operative Vorgehensweise wurde bei 9 Kieferhöhlen mit insgesamt 25 Implantaten durchgeführt. Die mittlere Beobachtungszeit betrug 78 Monate (Min. 3 Monate, Max. 166 Monate).

Folgende Fragestellungen wurden bei der Auswertung der Ergebnisse berücksichtigt:

1. Wie groß war der Unterschied zwischen der präoperativen vertikalen Knochenhöhe bei der einzeitigen gegenüber der zweizeitigen Operationsmethode?

2. Welche postoperative vertikale Augmentathöhe (ATH) wurde erreicht?

3. Wie ist die Verteilung der Implantate auf die verschiedenen Zahnregionen?

4. Wie groß ist die Implantatverlustrate?

5. Wie groß ist der Knochenverlust an den Implantaten?

6. Welche Komplikationen wurden beobachtet und gab es dadurch Misserfolge? 


\section{1 Überlebenszeit der Implantate}

Die kumulative Überlebensrate (Abb. 5) nach der mittleren Beobachtungszeit von 78 Monaten betrug $95,8 \%$.

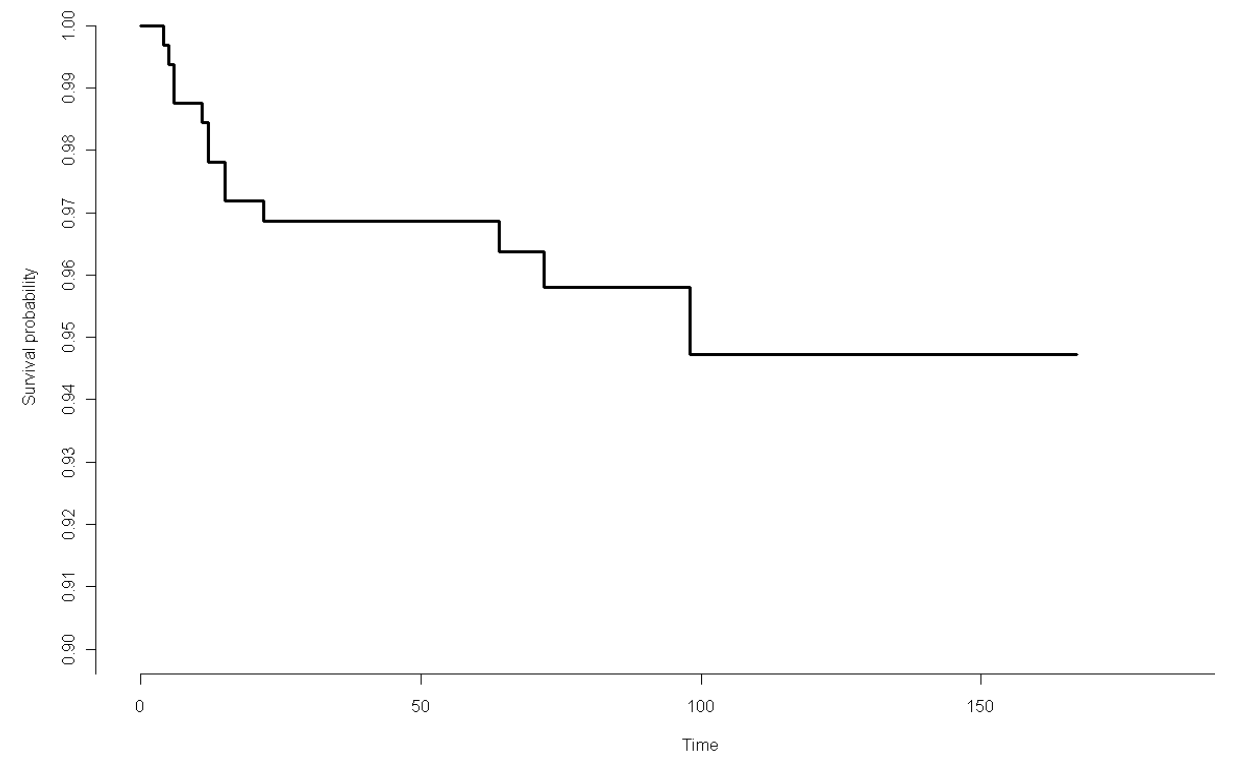

Abbildung 5

Die Überlebensrate getrennt nach Implantatsystemen (Abb. 6) ergab für die Frialit-2Implantate ( $\mathrm{n}=36) 100 \%$, für die Semados-Implantate $(\mathrm{n}=116) 98,3 \%$, für die Xive-Implantate $(\mathrm{n}=118)$ 95,7\% und bei den ITI-Implantaten $(\mathrm{n}=50) 88,8 \%$.

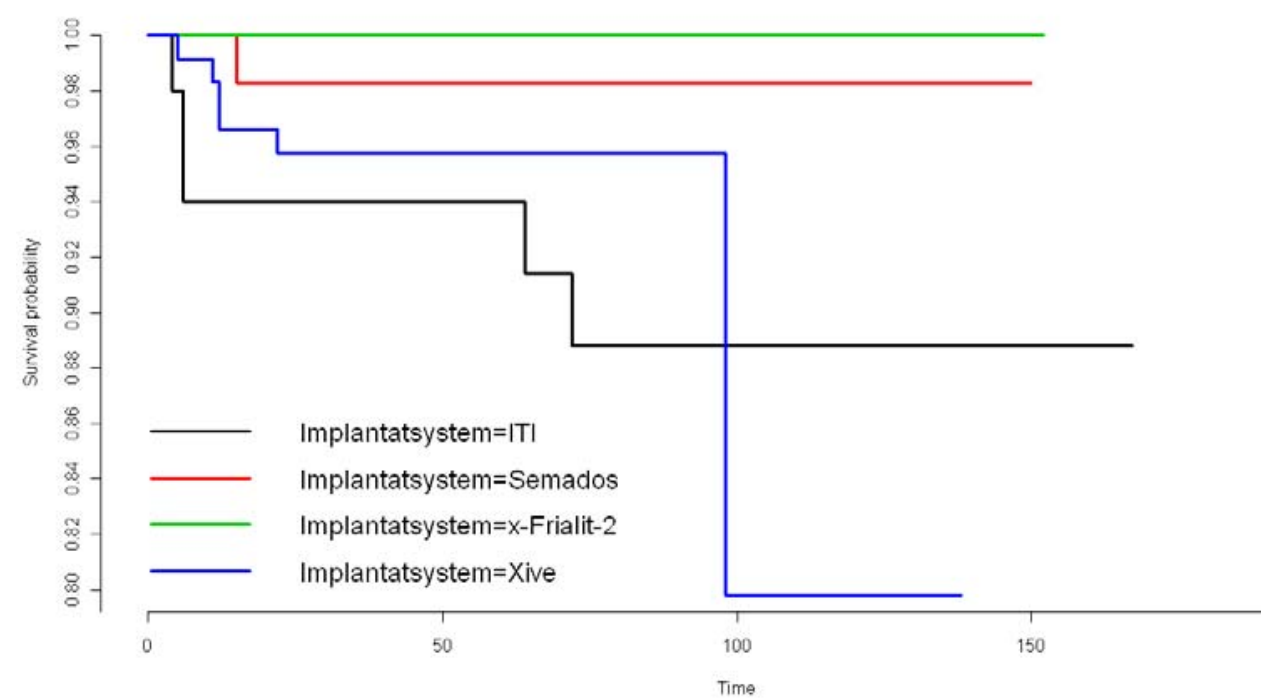

Abbildung 6 
Getrennt nach der Implantatposition im Kiefer (Abb. 7) betrug die Überlebensrate für die Implantate in der Region des ersten Prämolaren (4er, n=94) 96,9\%, für die Region des zweiten Prämolaren (5er, n=107) 97,2\%, für die Region des ersten Molaren (6er, $n=90)$ 92,6\% und für die Region des zweiten Molaren (7er, n=29) 96,4\%.

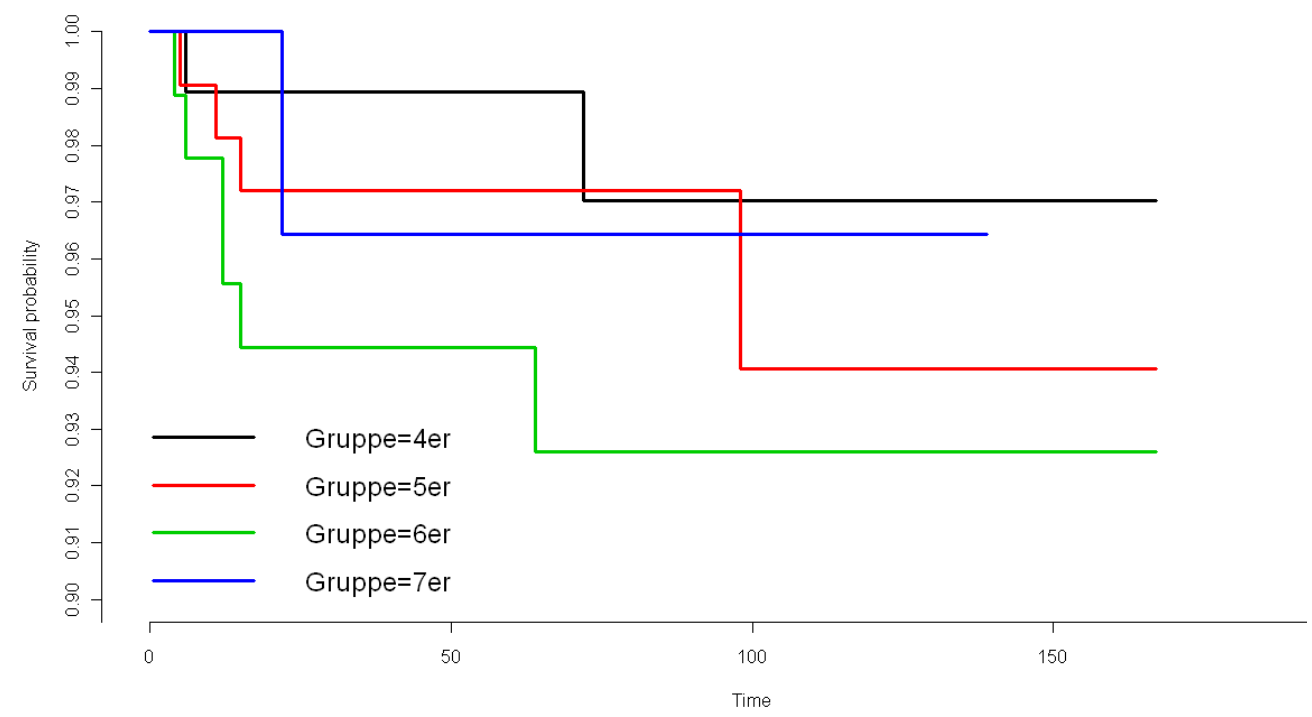

Abbildung 7

Die Überlebenswahrscheinlichkeit der Implantate unterschieden nach Augmentationsmaterial (Abb. 8) ergab für den autologen Knochen ( $n=54$ ) 90,1\% und für alloplastisches Material $(n=266)$ 97,4\%.

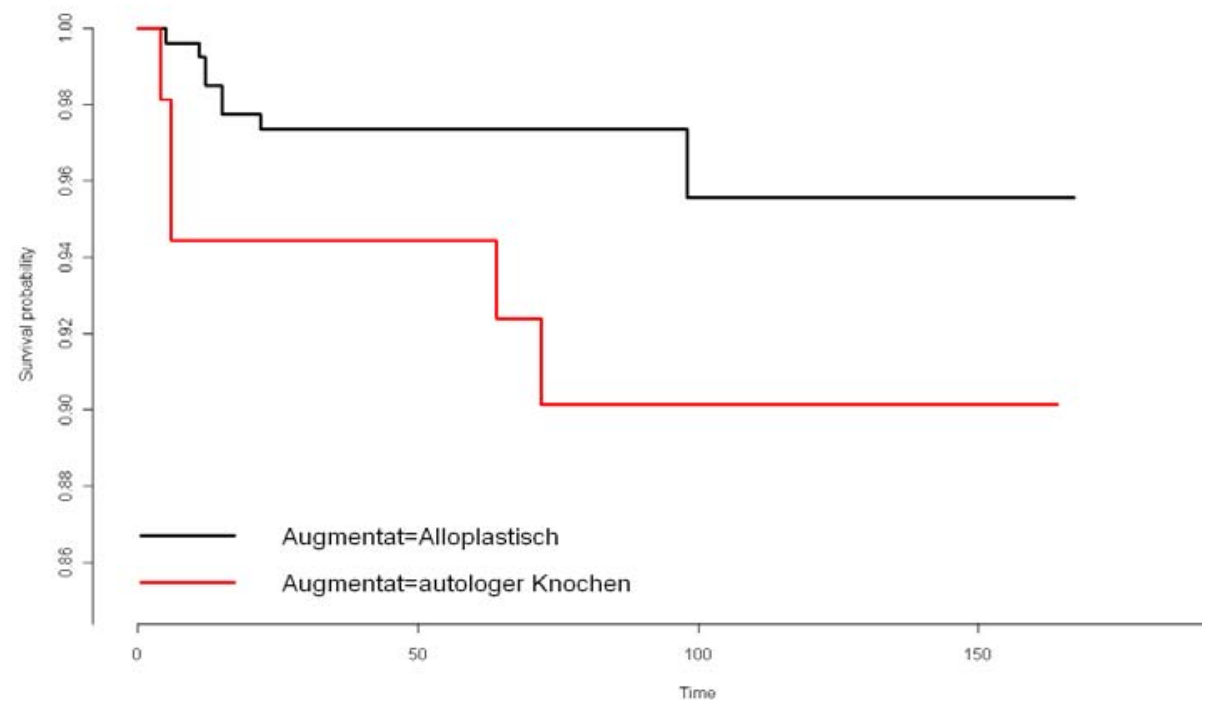

Abbildung 8 
Die Überlebensrate für Implantate, die in einem einzeitigen Operationsverfahren $(n=295)$ inseriert wurden betrug 96,2\%. Beim zweizeitigen Verfahren $(\mathrm{n}=25)$ konnte eine Überlebensrate von 92,0\% ermittelt werden (Abb. 9).

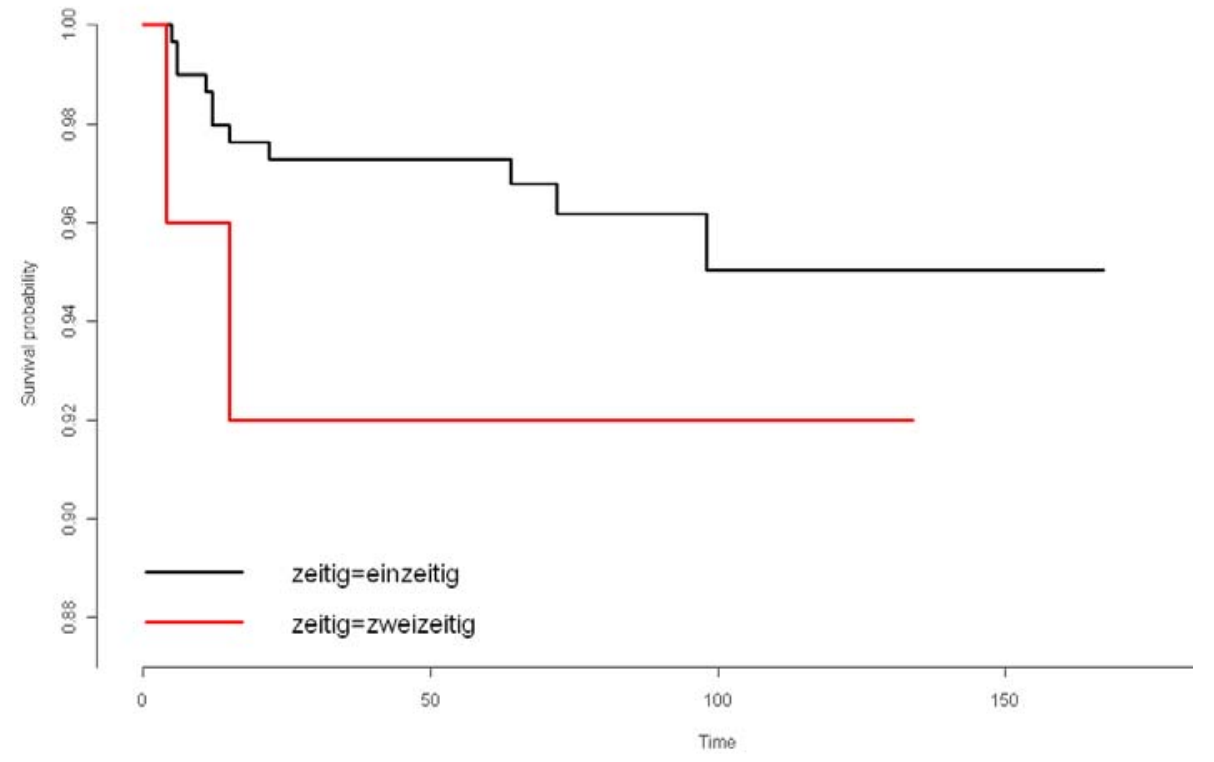

Abbildung 9

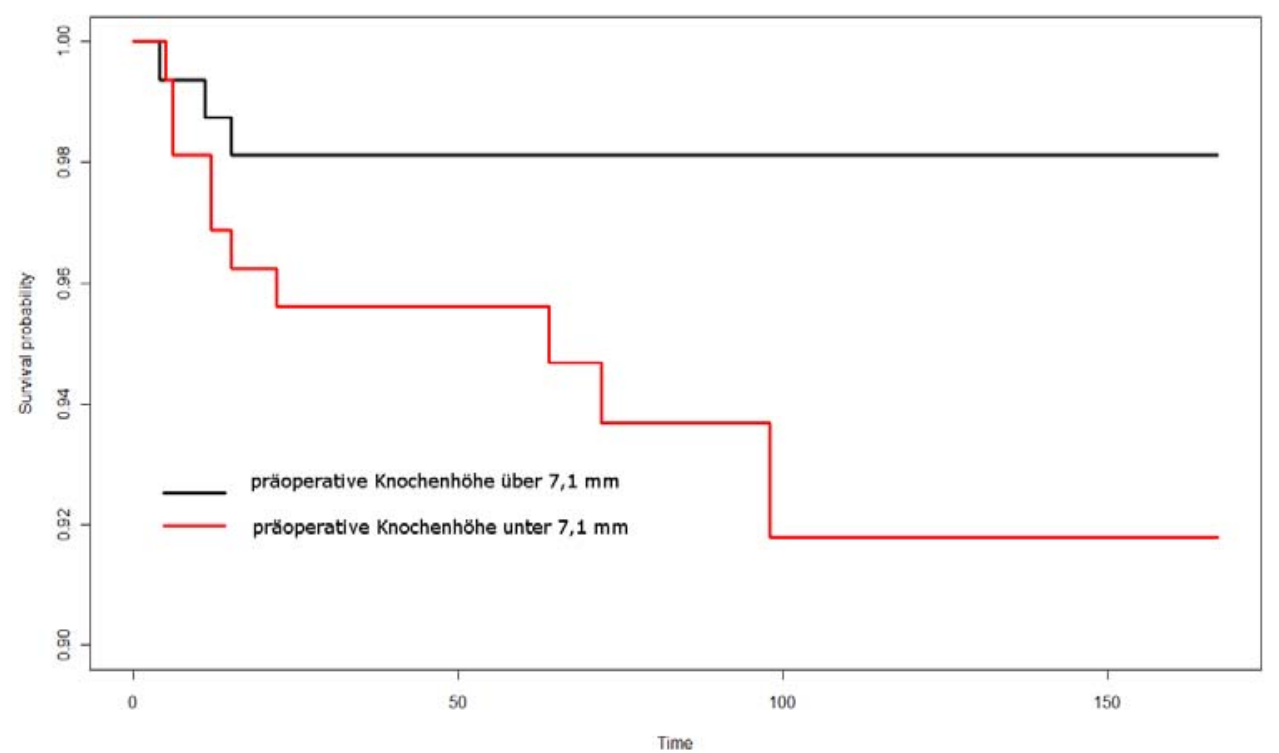

Abbildung 10 
Abbildung 10 (S. 37) zeigt die Überlebensrate für die Ausgangsknochenhöhe. Die präoperative Knochenhöhe wurde beim Median von 7,1 in zwei Gruppen unterteilt. Für geringere Ausgangsknochenhöhen konnte eine Überlebensrate von 93,6\% ermittelt werden. Für größere präoperative Knochenhöhen betrug die Überlebensrate 98,2\%. Für den präoperativen Knochen bedeutet dies: Steigt der präoperative Knochen um $1 \mathrm{~mm}$, dann ist die Verlustrate um den Faktor 0.812 kleiner, beziehungsweise: ist die präoperative Knochenhöhe $1 \mathrm{~mm}$ kleiner, dann ist die Verlustrate um den Faktor 1/0.812 = 1.23 größer.

\subsection{Erfolgsrate der Implantate}

Unter Anwendung der Erfolgskriterien von Jahn und d'Hoedt (1992) zum maximalen Beobachtungszeitraum (166 Monate) wurde eine Erfolgsrate von 94,7\% ermittelt. Die Anwendung der Kriterien von Naert et al. (1992) und Snauwaert et al. (2000) ergab eine Erfolgsrate von 94,4\%.

\begin{tabular}{|l|l|}
\hline Jahn und d'Hoedt (1992) & n Misserfolge \\
\hline Implantat in situ & $\begin{array}{l}\text { 13 Implantat- } \\
\text { verluste }\end{array}$ \\
\hline $\begin{array}{l}\text { Lockerungsgrade 0-1(entspricht } \\
\text { Periotestwert -8 bis +9) }\end{array}$ & 0 \\
\hline $\begin{array}{l}\text { Das Implantat darf keinen } \\
\text { zweiseitig durchgehenden Spalt } \\
\text { mit einer Breite größer als 0,5mm } \\
\text { in Röntgenbild aufweisen }\end{array}$ & 0 \\
\hline $\begin{array}{l}\text { Der anguläre Knochendefekt } \\
\text { (Mittelwert der mesialen und } \\
\text { distalen Messung am } \\
\text { Röntgenbild) darf nicht mehr als } \\
\text { 3/10 des konstruktiv enossalen } \\
\text { Implantatabschnittes betragen }\end{array}$ & 0 \\
\hline $\begin{array}{l}\text { Die Sulkustiefe darf nicht mehr } \\
\text { als 4mm bei zwei aufeinander } \\
\text { folgenden Kontrollen betragen }(*)\end{array}$ & Nicht bewertet \\
\hline $\begin{array}{l}\text { Die subjektive Bewertung des } \\
\text { Implantates durch den Patienten } \\
\text { darf nicht schlechter als 3 sein } \\
\text { (deutsches Schulnotensystem) }\end{array}$ & 4 (Note 4 = \\
\hline $\begin{array}{l}\text { Gesamte Misserfolge nach } \\
\text { Jahn und d'Hoedt (1992) }\end{array}$ & $\mathbf{1 7}$ \\
\hline Erfolgsquote & $\mathbf{9 4 , 7 \%}$ \\
\hline
\end{tabular}

\begin{tabular}{|l|l|}
\hline $\begin{array}{l}\text { Naert et al. (1992) } \\
\text { Snauwaert et al. (2000) }\end{array}$ & n Misserfolge \\
\hline $\begin{array}{l}\text { Implantat in situ und } \\
\text { prothetisch versorgt }\end{array}$ & $\begin{array}{l}\text { 13 Implantat- } \\
\text { verluste }\end{array}$ \\
\hline Periotestwert $<+8$ & 0 \\
\hline $\begin{array}{l}\text { Keine periimplantäre } \\
\text { Radiotransluzenz }\end{array}$ & 0 \\
\hline Keine Implantatfraktur & 0 \\
\hline $\begin{array}{l}\text { Keine Implantatinduzierten } \\
\text { Schmerzen, Infektionen } \\
\text { oder Parästhesien }\end{array}$ & 5 \\
\hline $\begin{array}{l}\text { Gesamte Misserfolge nach } \\
\text { Naert et al. (1992) und } \\
\text { Snauwaert et al. (2000) }\end{array}$ & 18 \\
\hline Erfolgsquote & $\mathbf{9 4 , 4 \%}$ \\
\hline
\end{tabular}

\section{Tabelle 6}

(*) wurde in dieser Studie nicht berücksichtigt 


\subsection{Verteilung der Implantate auf die verschiedenen Zahnregionen}

Insgesamt wurden 320 Implantate inseriert. Die Anzahl der gesetzten Implantate sind in der Region des ersten Prämolaren 94, in der Region des zweiten Prämolaren 107, in der ersten Molarenregion 90, in der zweiten Molarenregion 29.

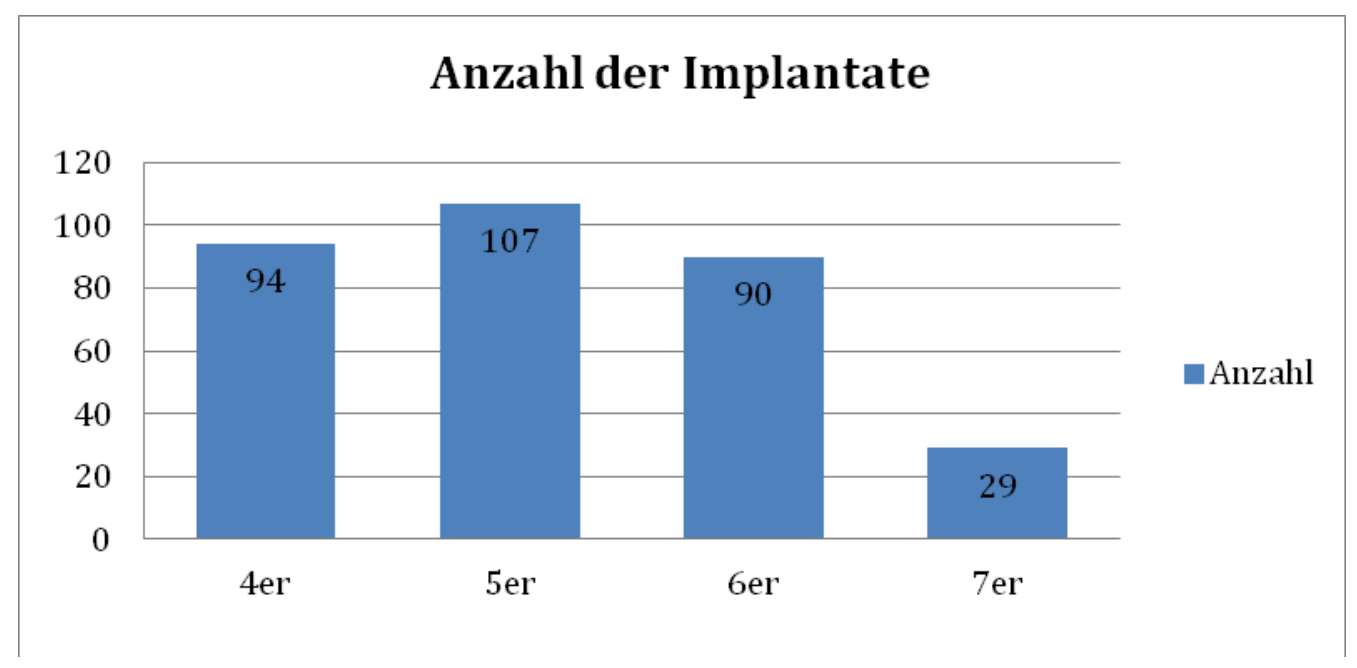

Abbildung 11

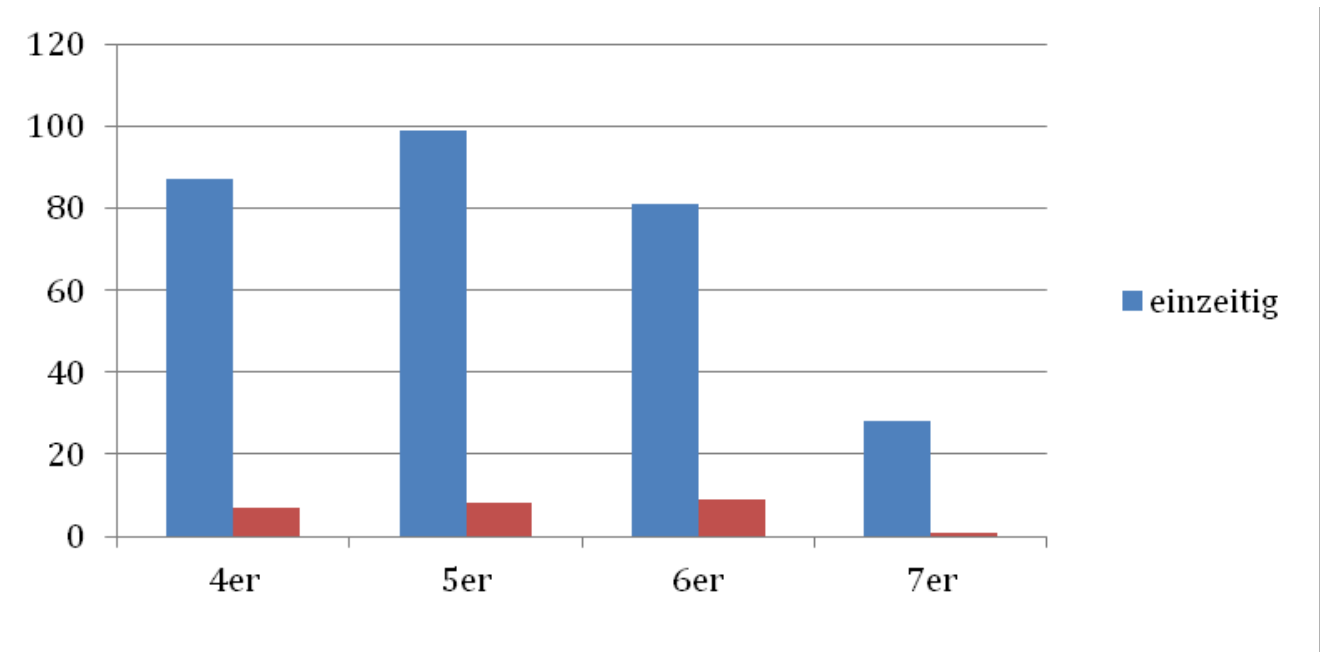

\section{Abbildung 12}

Beim einzeitigen Operationsverfahren wurden an Position des ersten Prämolaren 87 Implantate, beim zweiten Prämolaren 99, beim ersten Molaren 81 und beim zweiten Molaren 28 Implantate inseriert. Beim zweizeitigen Verfahren wurden an Position des 4ers sieben Implantate, an Position des 5ers acht Implantate, beim 6er neun Implantate und beim 7er ein Implantat gesetzt. 


\subsection{Implantatverluste}

Von den 320 inserierten Implantaten gingen im gesamten Beobachtungszeitraum 13 Implantate verloren. Acht der 13 verloren gegangenen Implantate gehörten dem männlichen Patientenkollektiv an, die restlichen fünf dem weiblichen. Bei der einzeitigen Operationsmethode gingen von insgesamt 295 Implantaten 11 verloren. Der maximale Beobachtungszeitraum betrug 166 Monate.

Bei der zweizeitigen Operationsmethode gingen von 25 Implantaten zwei verloren. Der maximale Beobachtungszeitraum betrug 133 Monate.

Sieben der Implantate gingen innerhalb des ersten Jahres nach Implantation verloren, weitere drei innerhalb des zweiten Jahres. Spätere Implantatverluste ereigneten sich nach fünf, sechs und acht Jahren.

Die Verluste der Implantate teilen sich wie folgt auf die verschiedenen Zahnregionen auf:

Regio 4=2; Regio 5=4; Regio 6=6; Regio 7=1

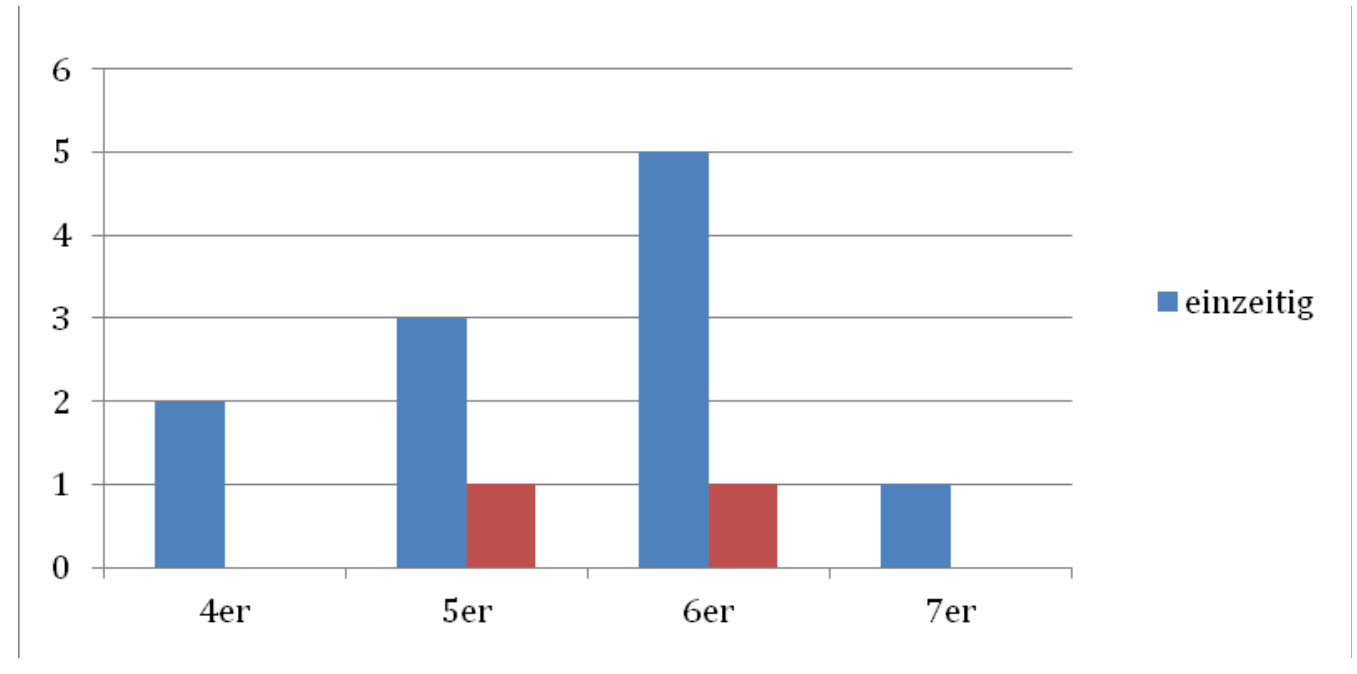

Abbildung 13 


\subsection{Präoperative vertikale Knochenhöhen}

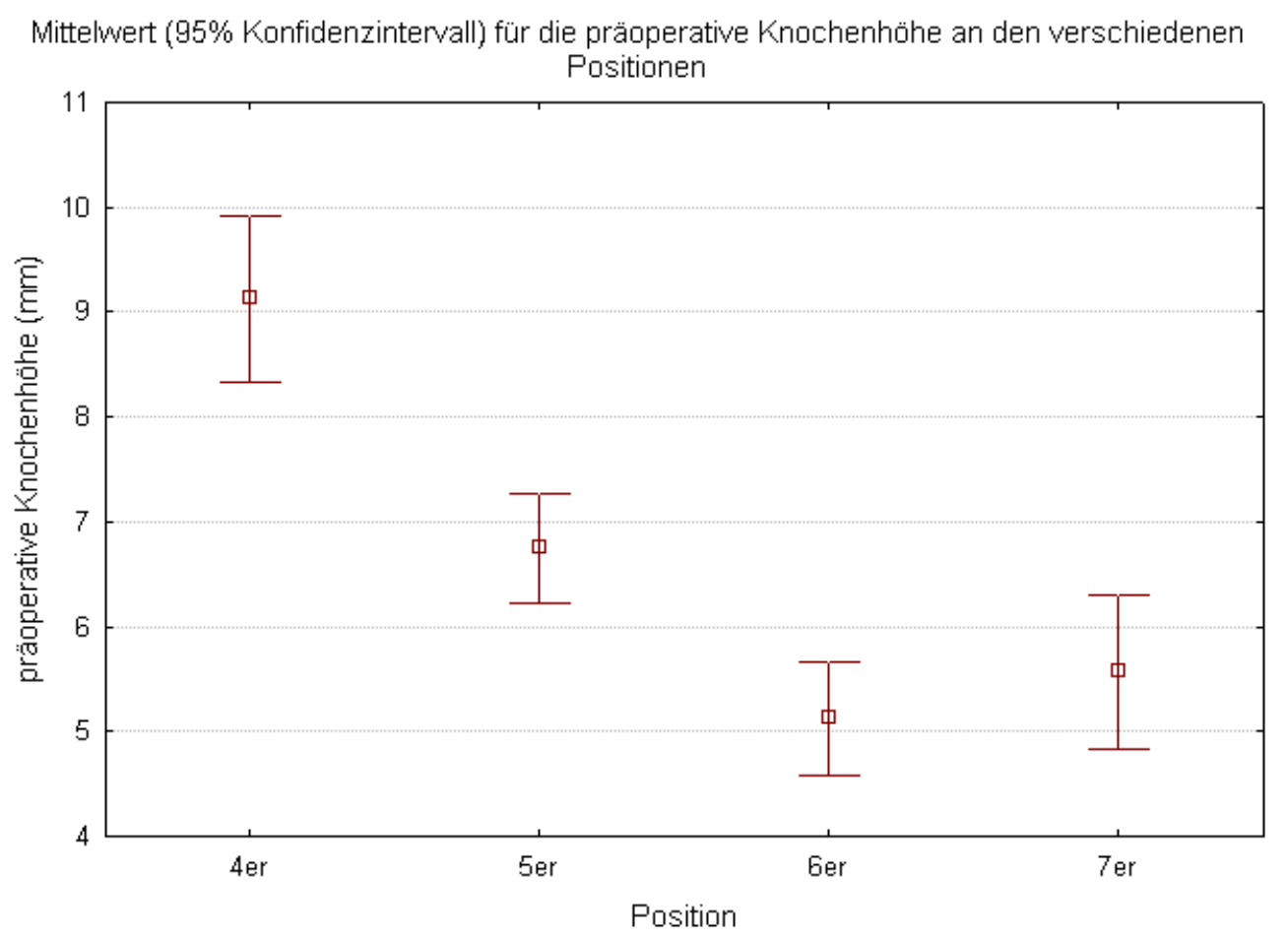

\section{Abbildung 14}

Die mittlere präoperative Knochenhöhe der Patienten betrug 6,9 mm (Min. 0,8 mm, Max. 19,2 mm, S.A. 3,3). Getrennt nach Zahnregionen ergeben sich für die Region des ersten Prämolaren (n= 94) 9,1 mm, für die zweite Prämolarenregion $(\mathrm{n}=107)$ 6,8 mm, für die Region des ersten Molaren ( $\mathrm{n}=90)$ 5,1 mm, für die Region des zweiten Molaren $(\mathrm{n}=29) 5,6 \mathrm{~mm}$.

\section{Vertikale Knochenhöhen der einzeitigen SALSA:}

Die mittlere präoperative Knochenhöhe der Patienten der einzeitigen SALSA betrug 7,0 mm (Min. 0,8 mm, Max. 19,2 mm, S.A. 3,4). Getrennt nach Zahnregionen ergeben sich für das weibliche Geschlecht für die Region des ersten Prämolaren $(n=46) 8,8 \mathrm{~mm}$, für die zweite Prämolarenregion $(\mathrm{n}=55)$ 6,3 mm, für die Region des ersten Molaren $(\mathrm{n}=49) 5,1 \mathrm{~mm}$, für die Region des zweiten Molaren ( $\mathrm{n}=19)$ 5,4 mm.

Getrennt nach Zahnregionen ergeben sich für das männliche Geschlecht für die Region des ersten Prämolaren ( $n=43)$ 10,0 mm, für die zweite Prämolarenregion $(n=44) 7,5 \mathrm{~mm}$, für die Region des ersten Molaren $(n=32)$ 5,2 mm, für die Region des zweiten Molaren $(n=9) 5,7$ $\mathrm{mm}$. 
Vertikale Knochenhöhen der zweizeitigen SALSA:

Die durchschnittliche präoperative Knochenhöhe bei der zweizeitigen SALSA betrug 5,6 mm (Min. 1,2 mm, Max. 10,4 mm, S.A. 2,4). Getrennt nach Zahnregionen ergeben sich für das weibliche Geschlecht für die erste Prämolarenregion $(n=7) 5,8 \mathrm{~mm}$, für die zweite Prämolarenregion ( $\mathrm{n}=7$ ) 5,8 mm, für die Region des ersten Molaren $(\mathrm{n}=7)$ 5,9 mm und für die Region des zweiten Molaren ( $\mathrm{n}=1$ ) 7,2 mm.

Getrennt nach Zahnregionen ergeben sich für das männliche Geschlecht für die zweite Prämolarenregion ( $\mathrm{n}=1$ ) 6,4 mm und für die Region des ersten Molaren $(\mathrm{n}=2)$ 1,8 mm. An den Positionen des ersten Prämolaren und des zweiten Molaren wurden keine Implantate im zweizeitigen Vorgehen gesetzt.

\subsection{Postoperative vertikale Augmentathöhen}

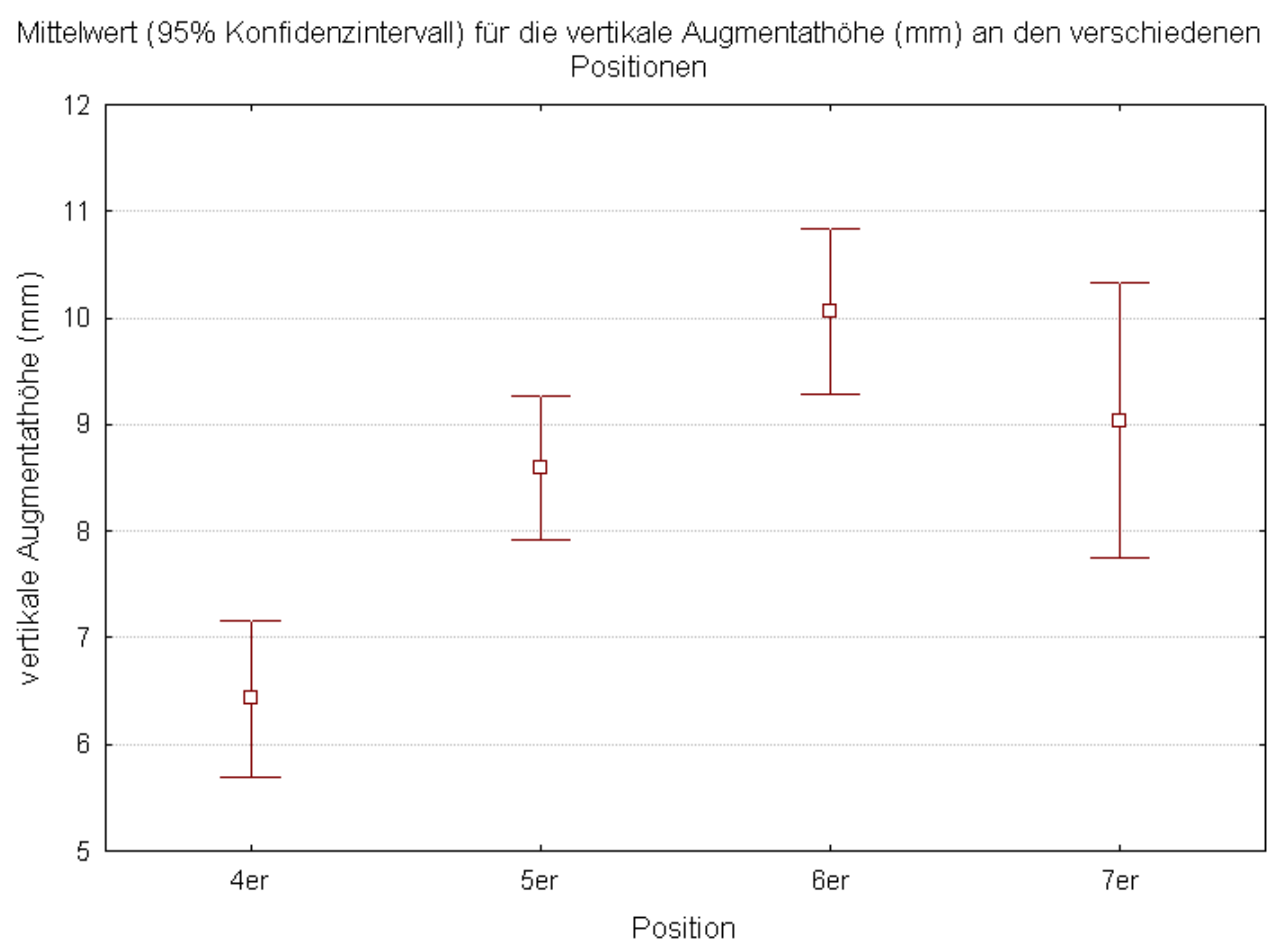

\section{Abbildung 15}

Je weiter distal sich die Implantate im Kiefer befinden, desto größer ist die vertikale Augmentathöhe. Die mittlere vertikale Augmentathöhe betrug 8,4 mm (Min. 0 mm, Max. 18,4 mm, S.A. 3,8). Getrennt nach Zahnregionen ergeben sich für die Position des ersten Prämolaren 6,4 $\mathrm{mm}$, beim zweiten Prämolaren $8,6 \mathrm{~mm}$, beim ersten Molaren 10,1 $\mathrm{mm}$ und beim zweiten Molaren 9,0 mm. 


\section{Vertikale erreichte Augmentathöhen bei der einzeitigen SALSA:}

Die durchschnittliche postoperativ erreichte vertikale ATH bei der einzeitigen SALSA betrug 8,4 mm (Min. 0 mm, Max. 18,4 mm, S.A. 3,8). Getrennt nach Zahnregionen ergeben sich für das weibliche Geschlecht für die erste Prämolarenregion $6,8 \mathrm{~mm}$, für die zweite Prämolarenregion 8,7 mm, für die Region des ersten Molaren 9,7 mm und für die Region des zweiten Molaren 8,5 mm.

Getrennt nach Zahnregionen ergeben sich für das männliche Geschlecht für die erste Prämolarenregion 5,9 mm, für die zweite Prämolarenregion $8,5 \mathrm{~mm}$, für die Region des ersten Molaren 10,6 mm und für die Region des zweiten Molaren 10,4 mm.

Vertikale erreichte Augmentathöhen bei der zweizeitigen SALSA:

Die durchschnittlich erreichte vertikale ATH bei der zweizeitigen SALSA betrug 8,6 mm (Min. $0 \mathrm{~mm}$, Max. 18,4 mm, S.A. 3,8). Getrennt nach Zahnregionen ergeben sich für das weibliche Geschlecht für die erste Prämolarenregion $8,4 \mathrm{~mm}$, für die zweite Prämolarenregion $8,0 \mathrm{~mm}$, für die Region des ersten Molaren 8,7 mm und für die Region des zweiten Molaren 5,6 mm.

Getrennt nach Zahnregionen ergeben sich für das männliche Geschlecht für die zweite Prämolarenregion 8,0 mm, für die Region des ersten Molaren 13,8 mm. 


\subsection{Periimplantäre Verhältnisse}

\section{Sulcus-Blutungs-Index}

Der Sulcus-Blutungs-Index betrug durchschnittlich 11\% (Min. 0\%, Max. 40\%). Getrennt nach Geschlechtern ergab sich für die Männer ein mittlerer SBI von 13,8\% (Min. 0\%, Max. 40\%, S.A. 10,5). Für das weibliche Geschlecht ergab sich ein SBI von 8,3\% (Min. 0\%, Max. 30\%, S.A. 8,3$)$.

\section{Sondierungstiefen}

Die mesiale Sondierungstiefe betrug durchschnittlich 2,9 mm (Min. $1 \mathrm{~mm}$, Max. $6 \mathrm{~mm}$, S.A. 0,84). Getrennt nach Zahnregion beträgt sie durchschnittlich in der ersten Prämolarenregion 2,8 mm, in der Region des zweiten Prämolaren 2,9 mm, in der Region des ersten Molaren 3,2 mm und in der Region des zweiten Molaren 3,2 mm. Je weiter distal im Oberkiefer die Implantate positioniert sind, desto höher ist der Wert der mesialen Sondierungstiefe am Implantat (siehe Abb. 16).

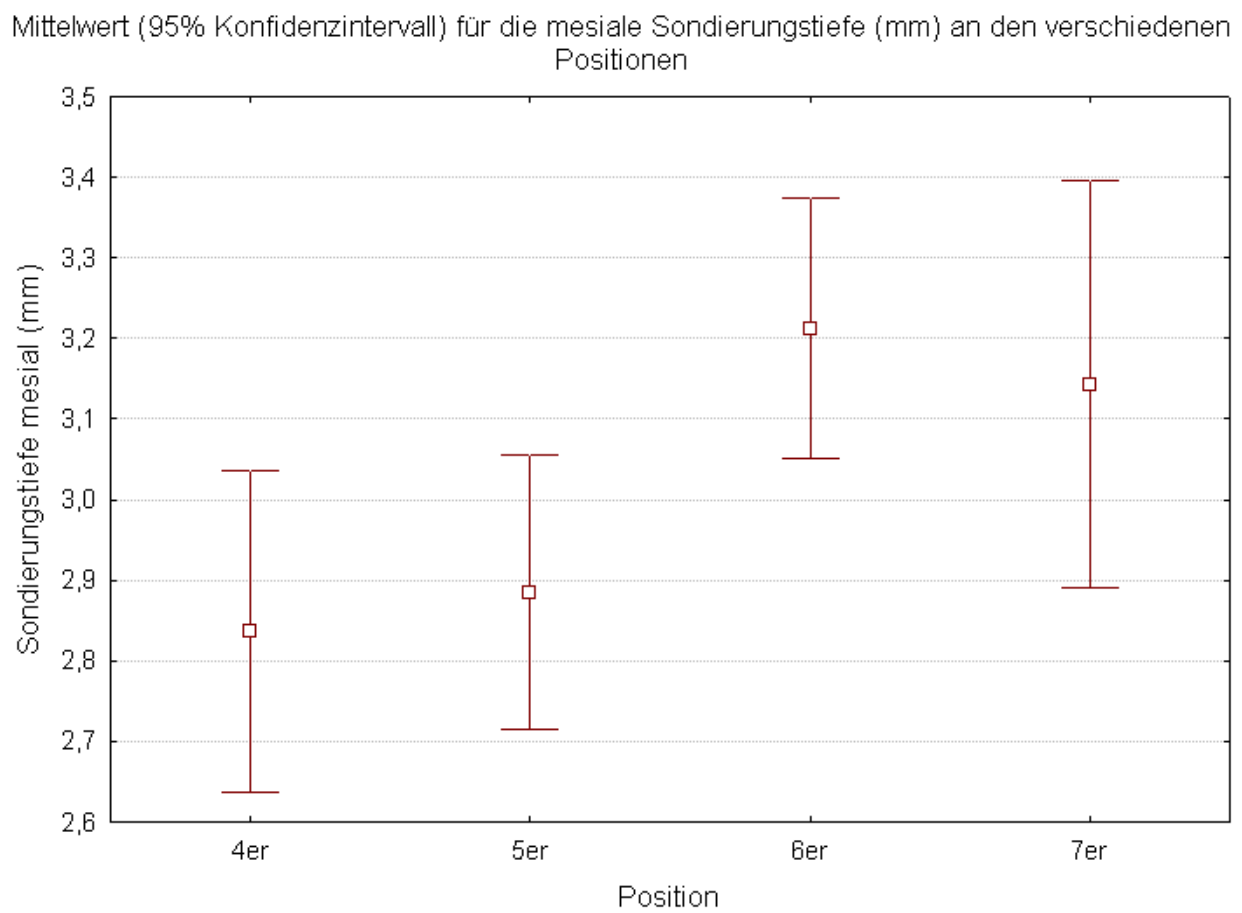

Abbildung 16 
Die distale Sondierungstiefe betrug durchschnittlich 3,3 mm ( Min. $1 \mathrm{~mm}$, Max. $5 \mathrm{~mm}$, S.A. 0,83). Getrennt nach Zahnregion beträgt die sie durchschnittlich in der ersten Prämolarenregion 2,9 mm, in der Region des zweiten Prämolaren 3,1 mm, in der Region des ersten Molaren 3,6 $\mathrm{mm}$ und in der Region des zweiten Molaren 3,8 mm. Ebenso wie die mesiale Sondierungstiefe, nimmt auch der Wert für die distale Sondierungstiefe zu, je weiter posterior die Implantatposition liegt (siehe Abb. 17).

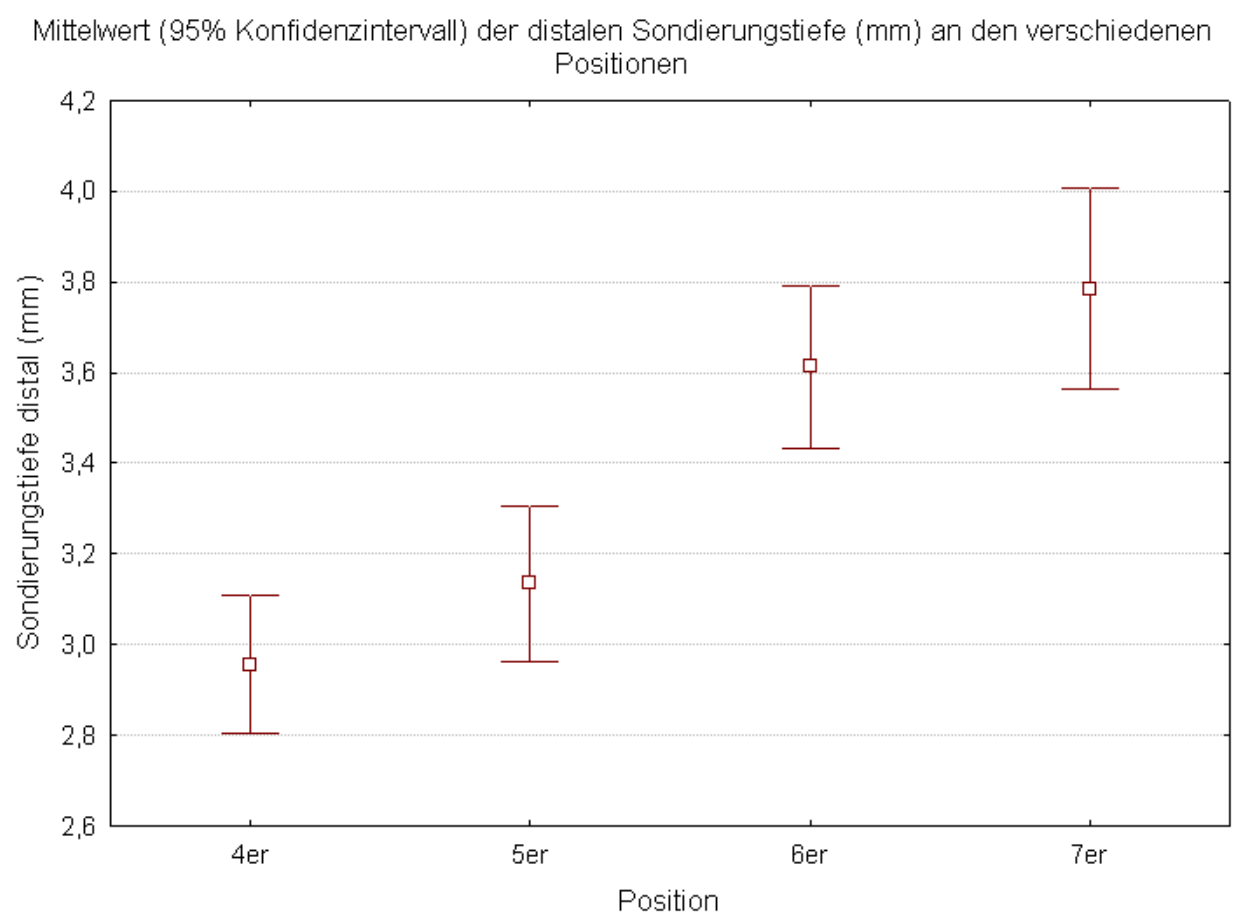

Abbildung 17

\section{Sondierungsbluten}

Es konnte ein signifikanter Einfluss des Alters der Patienten auf das Sondierungsbluten ermittelt werden $(p=0,0398)$. Mit zunehmendem Alter des Patienten steigt die Wahrscheinlichkeit für Bluten auf Sondieren an (Abb. 18). 


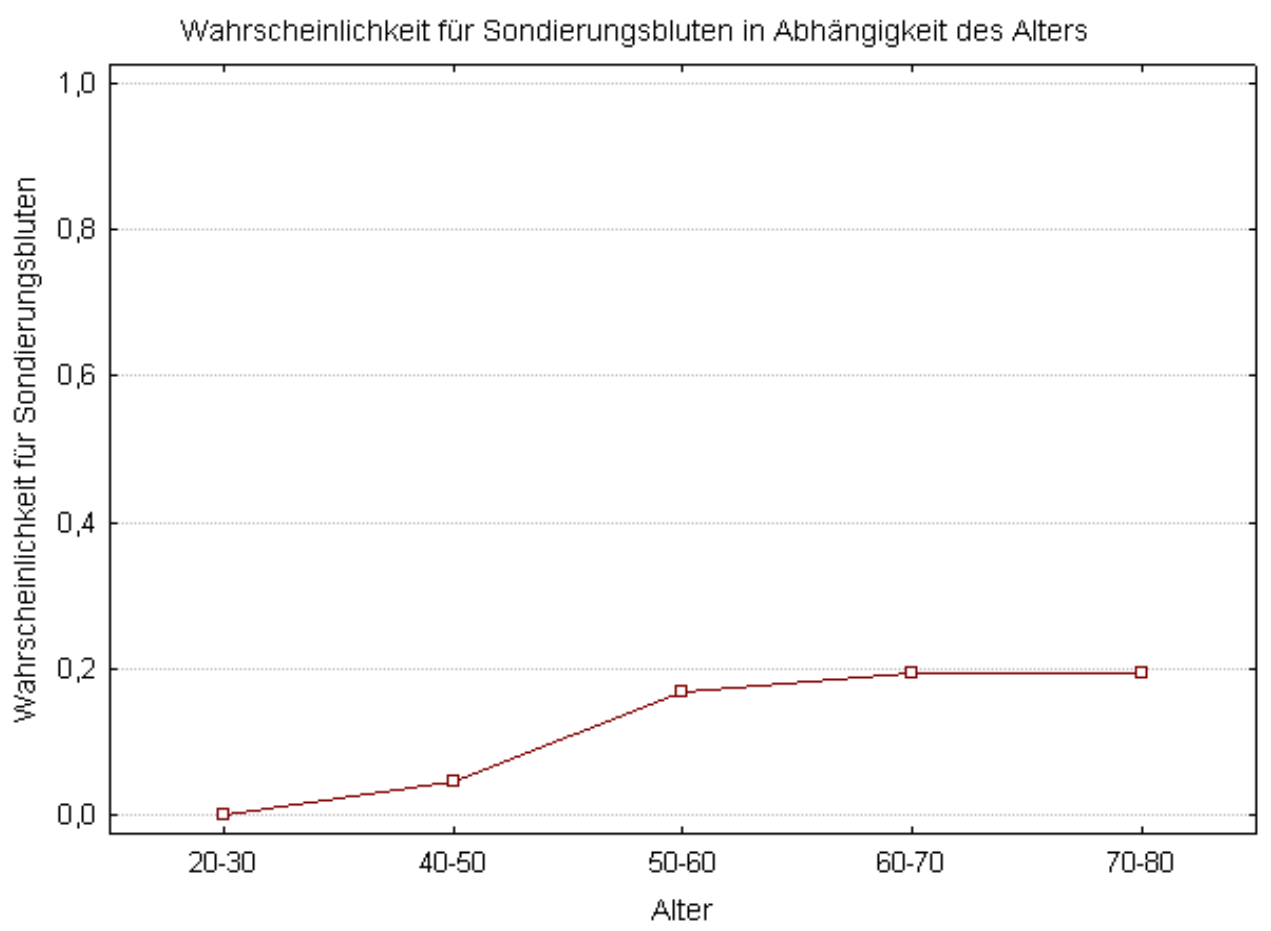

Abbildung 18

Wahrscheinlichkeit für Sondierungsbluten in Abhängigkeit der Anzahl der Implantate

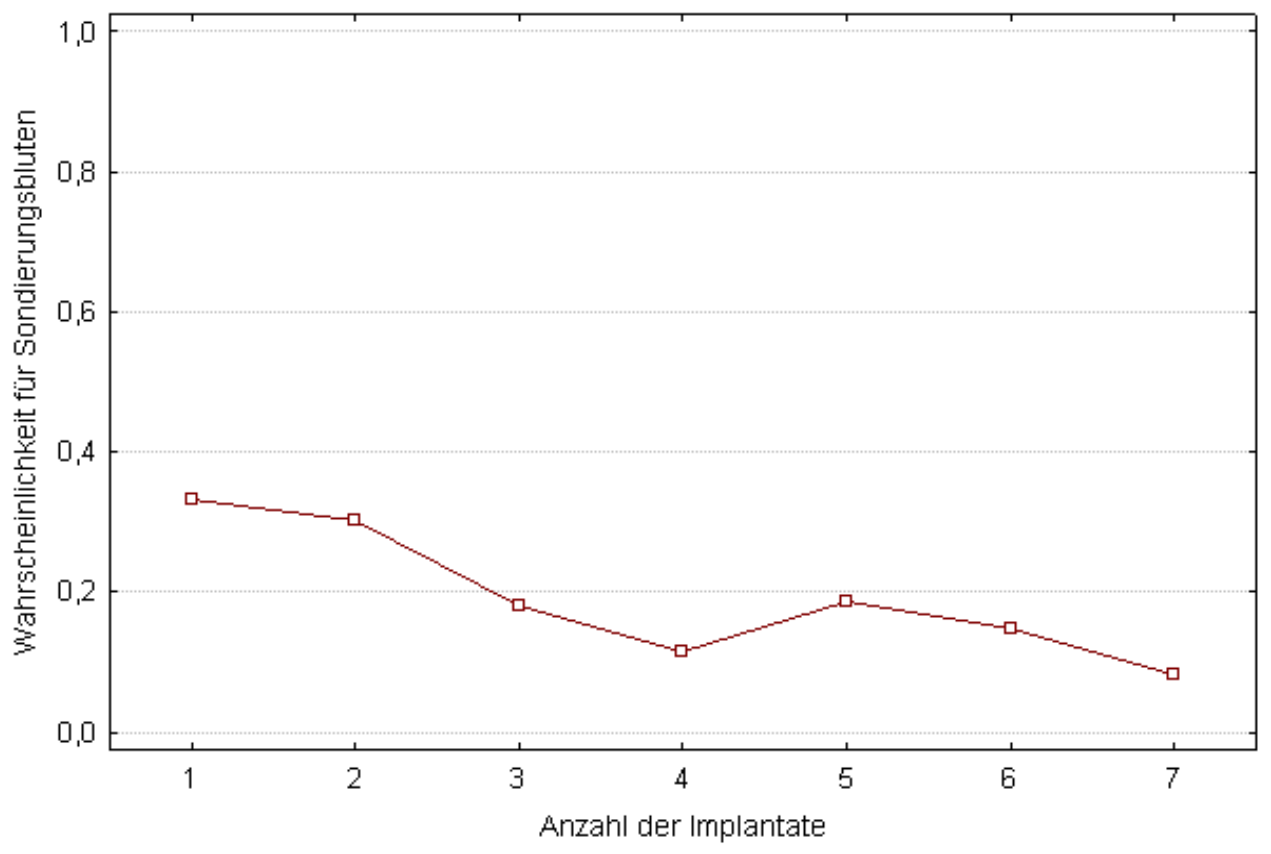

Abbildung 19

Abbildung 19 zeigt, dass die Wahrscheinlichkeit für Sondierungsbluten mit steigender Anzahl an Implantaten pro Patient tendenziell abnimmt. Bei 15,9\% $(\mathrm{n}=51)$ der Implantate konnte ein Bluten auf Sondieren festgestellt werden. 


\subsection{Periotest}

Der Periotest betrug durchschnittlich -2,42 (Min. -8, Max. 3,0, S.A. 2,2). Getrennt nach Zahnregion beträgt er durchschnittlich in der ersten Prämolarenregion -2,47, in der Region des zweiten Prämolaren -2,52, in der Region des ersten Molaren -2,19 und in der Region des zweiten Molaren -2,57.

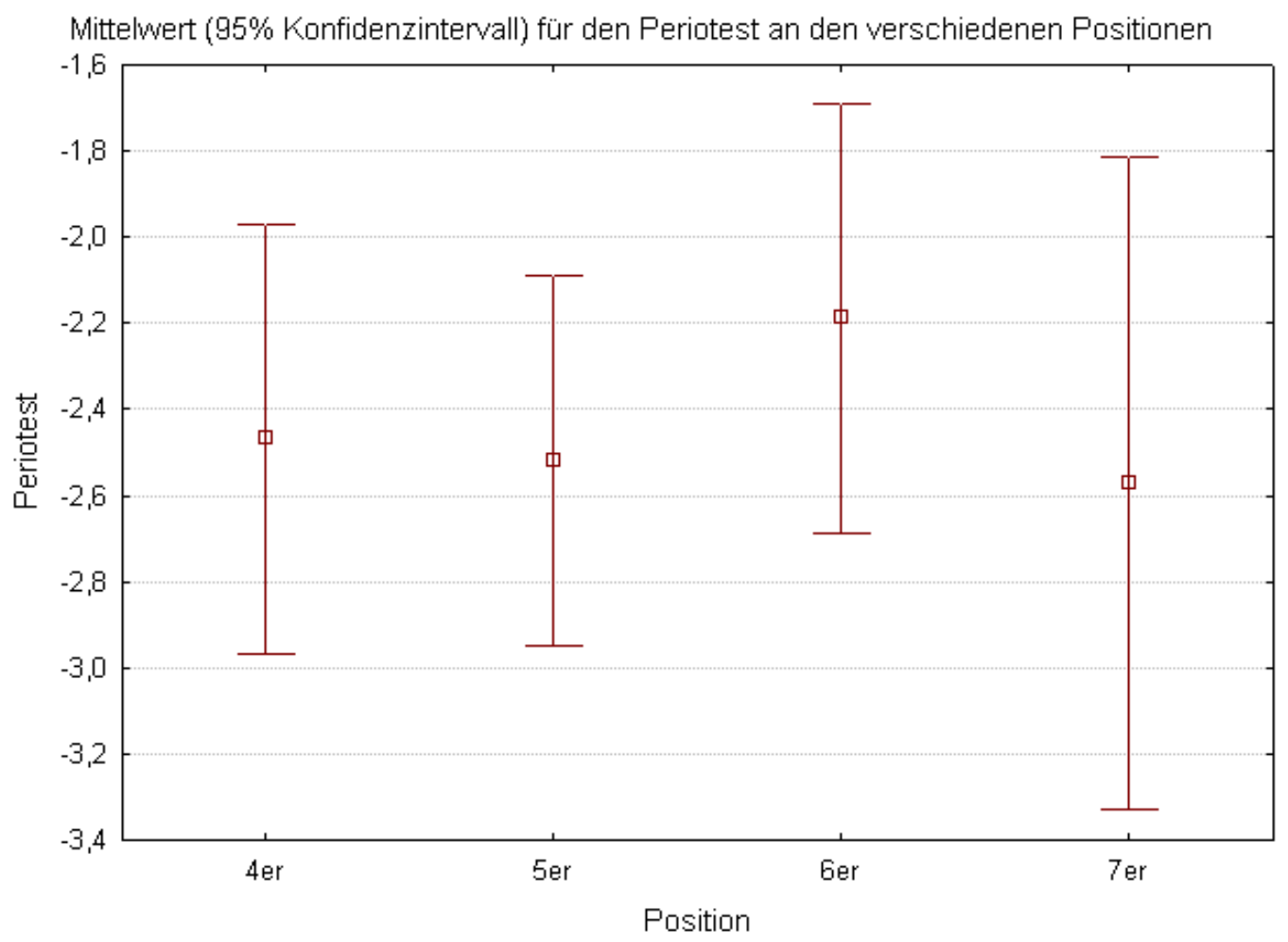

Abbildung 20

\subsection{Knochenverlust}


Der mittlere Knochenabbau beträgt 2,9 mm (Min. -2,4, Max. 8,0 mm, S.A. 2,0). Getrennt nach Zahnregion beträgt er durchschnittlich in der ersten Prämolarenregion 2,5 mm, in der Region des zweiten Prämolaren 3,0 mm, in der Region des ersten Molaren 3,3 mm und in der Region des zweiten Molaren 2,7 mm (Abb. 21).

\section{Einzeitiges Operationsverfahren:}

Der mittlere Knochenabbau beträgt 2,8 mm (Min. -2,4, Max. 8,0, S.A. 2,0). Getrennt nach Zahnregion beträgt er durchschnittlich in der ersten Prämolarenregion 2,5 mm, in der Region des zweiten Prämolaren 2,9 mm, in der Region des ersten Molaren 3,2 $\mathrm{mm}$ und in der Region des zweiten Molaren 2,5 mm.

\section{Zweizeitiges Operationsverfahren:}

Der mittlere Knochenabbau beträgt 3,2 mm (Min. 0 mm, Max. 8,0, S.A. 2,5). Getrennt nach Zahnregion beträgt er durchschnittlich in der ersten Prämolarenregion 2,0 mm, in der Region des zweiten Prämolaren 3,5 mm, in der Region des ersten Molaren 3,1 mm und in der Region des zweiten Molaren 2,4 mm.

Es ließ sich ein signifikanter Einfluss der Position der Implantate im Kiefer auf den Knochenverlust nachweisen $(\mathrm{p}=0,0453)$. Je weiter nach distal die Implantate im posterioren Oberkiefer inseriert wurden, desto größer ist der marginale Knochenabbau.

Durch die Analyse weiterer Faktoren wie Implantatsystem, Augmentationsmaterial, einzeitiges oder zweizeitiges Operationsverfahren und Alter der Patienten ließ sich kein signifikanter Einfluss auf den Knochenverlust nachweisen. 


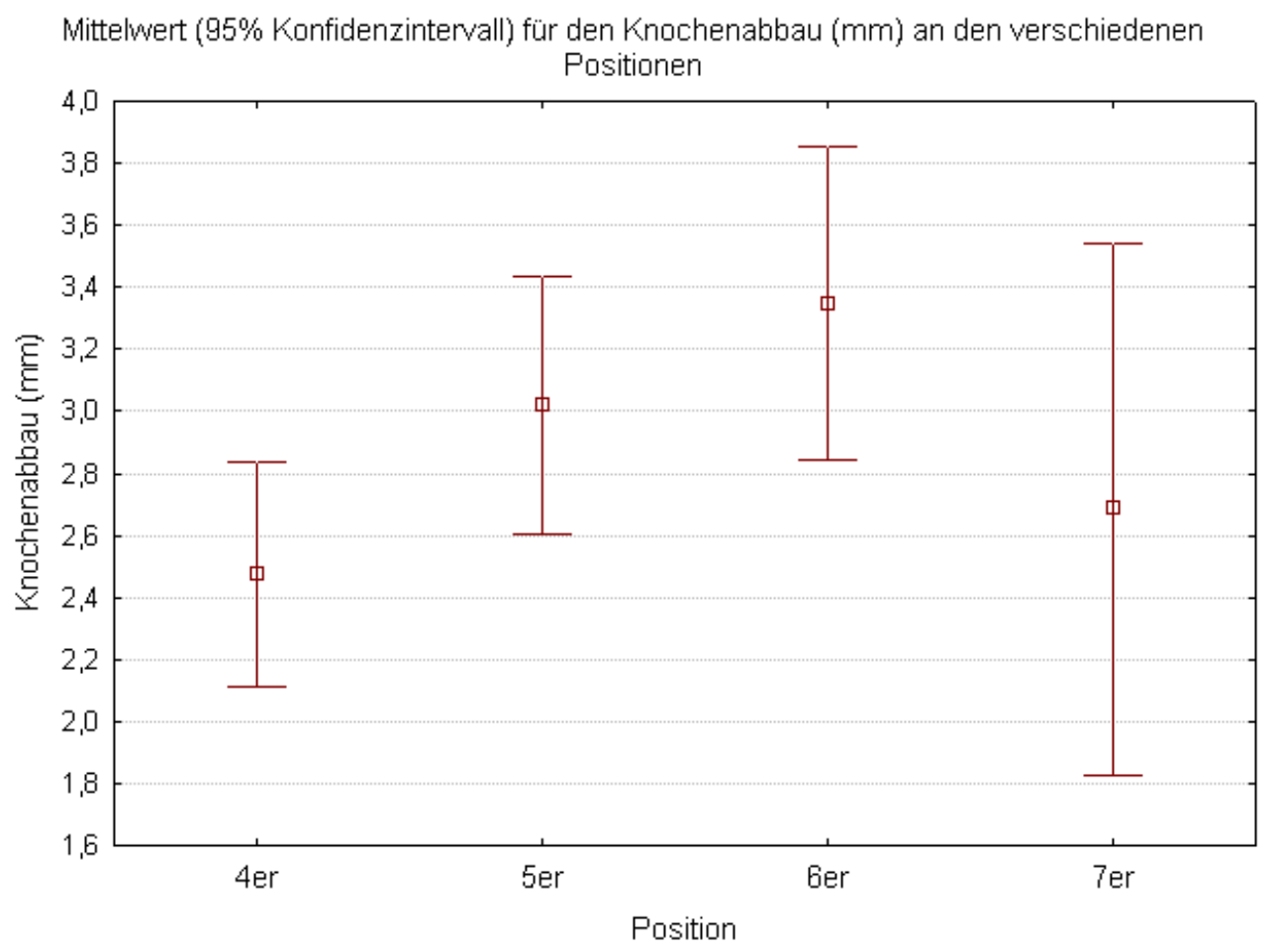

Abbildung 21

\section{Apikaler Knochenabbau:}

Der apikale Knochenabbau betrug durchschnittlich 1,5 mm (Min. 0,8 mm, Max. 3,2 mm, S.A. 0,6). Getrennt nach Zahnregion beträgt er durchschnittlich in der ersten Prämolarenregion 1,3 mm, in der Region des zweiten Prämolaren 1,6 mm, in der Region des ersten Molaren 1,4 mm und in der Region des zweiten Molaren 1,6 mm. Der mittlere apikale Knochenabbau lag beim einzeitigen Operationsverfahren bei 1,5 mm (Min. 0,8 mm, Max. 3,2 mm, S.A. 0,6). Für das zweizeitige Verfahren betrug der Durchschnittswert 1,2 mm (Min. 0,8 mm, Max. 1,6 mm, S.A. $0,5)$. 


\subsection{Fragebogen}

Es wurden insgesamt 94 vollständig ausgefüllte Fragebögen ausgewertet.

Frage 1: Wie beurteilen Sie das Gesamtresultat Ihrer Behandlung?

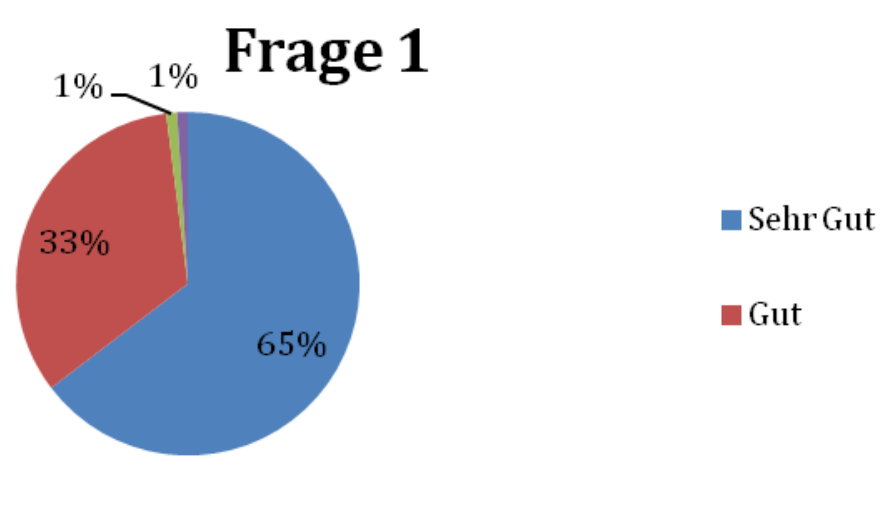

\section{Abbildung 22}

Die Zufriedenheitsanalyse auf der Skala des deutschen Schulnotensystems $(1=$ sehr gut bis $6=$ ungenügend) ergab, dass 65\% der Patienten mit einer 1 ( „sehr gut“), 33\% mit einer 2 (,gut“), jeweils 1\% der Patienten mit einer 3 ( ,befriedigend“) und einer 4 ( „ausreichend“) das gesamte Behandlungsresultat bewerteten.

Frage 2: Wie schätzen Sie den Halt Ihres Zahnersatzes ein?

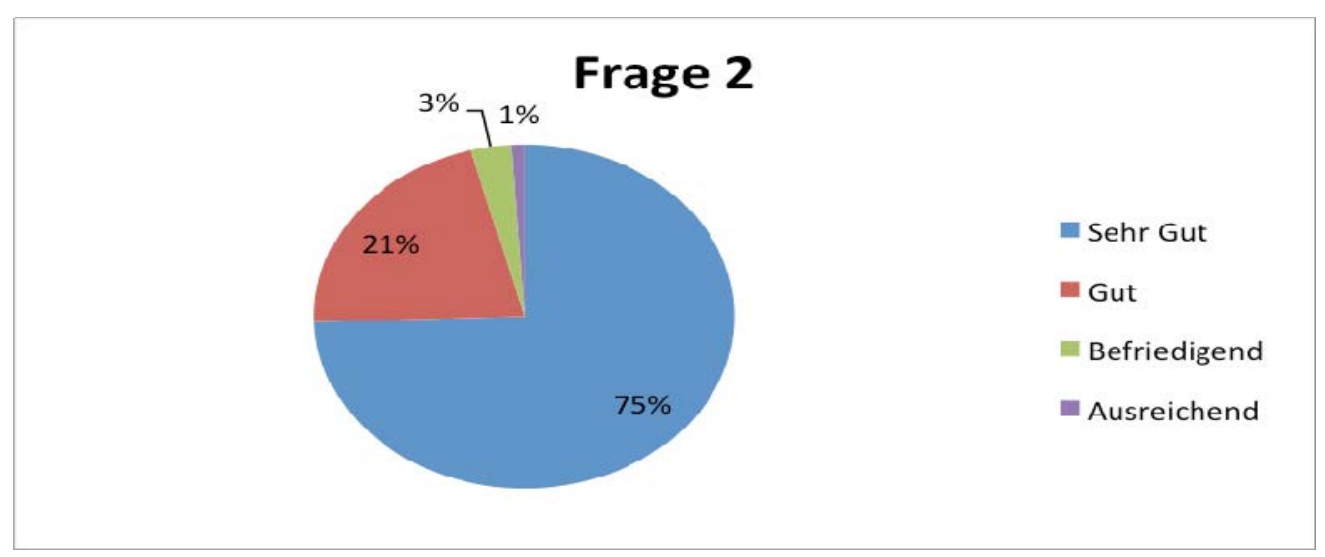

Abbildung 23

75\% der Patienten waren mit dem Halt Ihres Zahnersatzes sehr zufrieden und gaben die Note 1 („sehr gut"), $21 \%$ der Patienten beurteilten den Halt mit „gut“, 3\% mit „,befriedigend“ und lediglich $1 \%$ befand diesen als ,ausreichend“. 
Frage 3: Wie schätzen Sie Ihre Kaufunktion insgesamt ein?

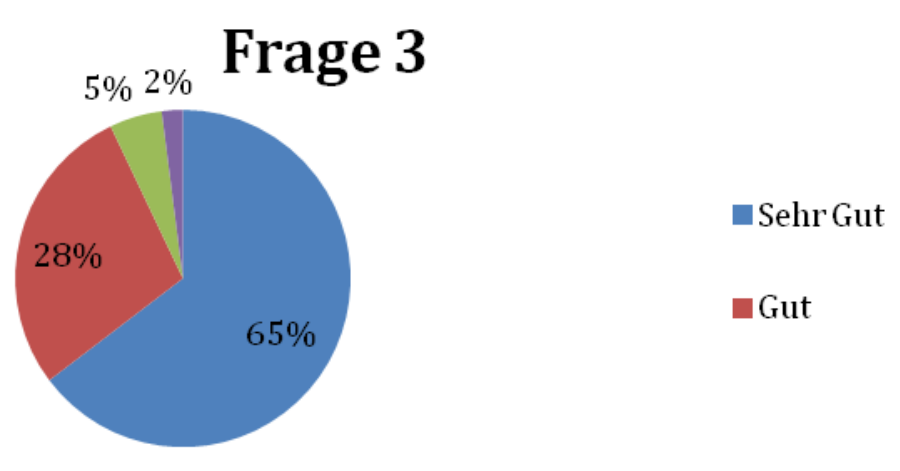

Abbildung 24

65\% der Patienten empfanden Ihre Kaufunktion nach Abschluß der chirurgischen und prothetischen Therapie als "sehr gut“, 28\% als „gut“, bei 5\% war die Kaufunktion „befriedigend“ und als ,ausreichend“ wurde sie bei $2 \%$ der Patienten beurteilt.

Frage 4: Wie oft mußten Sie auf Grund von Problemen oder Beschwerden mit Ihrem Zahnersatz einen Zahnarzt aufsuchen?

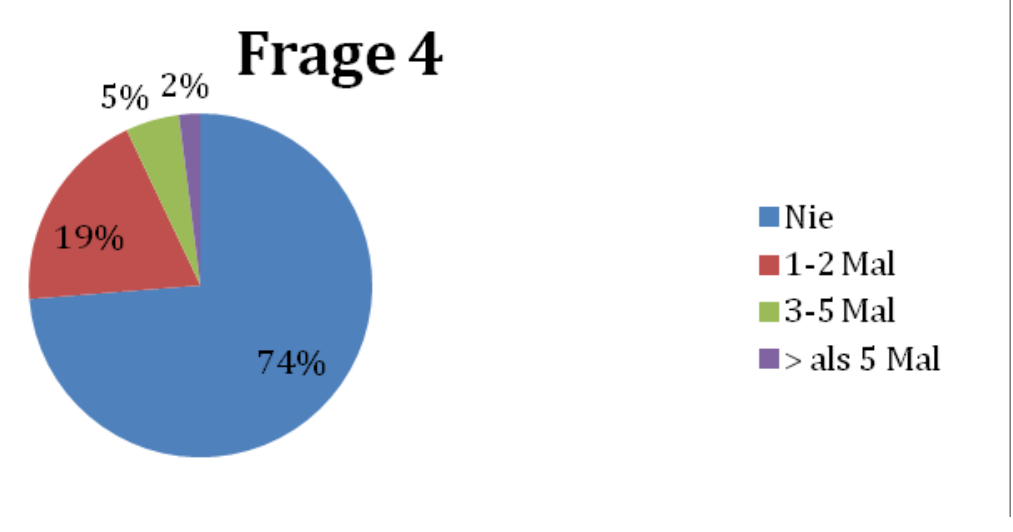

\section{Abbildung 25}

74\% der Patienten waren nach Abschluss der Therapie beschwerdefrei und mussten außer den regulären Kontrolluntersuchungen keinen Zahnarzt wegen Problemen mit dem Zahnersatz aufsuchen. 19\% waren 1-2 Mal zusätzlich beim Zahnarzt, 5\% der Patienten 3-5 Mal und 2\% häufiger als $5 \mathrm{Mal}$. 


\subsection{Komplikationen}

Intraoperative Komplikationen:

Die am häufigsten auftretende Komplikation ist die Perforation der Schneiderschen Membran. Intraoperativ wurden bei 18 Patienten Perforationen der Schneiderschen Membran festgestellt. In 17 Fällen war die linke Kieferhöhle, in acht Fällen die rechte Kieferhöhle betroffen. Beim einzeitigen Verfahren traten bei 15 Patienten Perforationen auf und beim zweizeitigen Verfahren traten bei drei Patienten Perforationen auf. Bei 11 Patienten wurden einseitig und bei sieben Patienten wurden beidseitig Perforationen entdeckt. Die Perforationsrate beträgt 18,8\%. Alle Perforationen wurden durch resorbierbare Vicrylnetze verschlossen. Zur Stabilisierung des dünnen SMP und um ein Einreißen zu verhindern, wurden in 54 Fällen aus prophylaktischen Gründen resorbierbare Membranen verwendet. In vier Fällen (jeweils zwei im 1. und zwei im 2. Quadranten), bei denen intraoperativ Perforationen festgestellt wurden, kam es zu Implantatmisserfolgen.

\section{Postoperative Komplikationen:}

Eine Periimplantitis wurde in zwei Fällen beobachtet, bei beiden Patienten war der erste Quadrant betroffen. Die Therapie der Taschentiefen von $>5 \mathrm{~mm}$ erfolgte mittels Laser.

Mehrere Patienten klagten über Schwellungen, Hämatome und Druckdolenzen im Bereich der Kieferhöhlen. In einem Fall kam es zu einer Nachblutung nach Freilegung der Implantate.

Bei zwei Patienten wurde eine Mund-Antrum-Verbindung festgestellt. Die Therapie erfolgte durch plastische Deckung, abschwellende Nasentropfen und Antibiotikagabe.

Als weitere Komplikation wurde eine Sinusitis maxillaris nach Sinuslift klinisch und radiologisch (Spiegelbildung) bei drei Patienten diagnostiziert. Die rein konservative Therapie erfolgte durch Clindamycingabe, abschwellende Nasentropfen und durch Kamilledampfbäder. Erhöhte Implantatverluste wurden nach diesen Komplikationen nicht gefunden. 


\subsection{Falldarstellung}

Die Patientin (66 Jahre) kam in die Universitätsklinik Göttingen mit Beschwerden im 2. Quadranten. Die Patientin trug eine Brücke von 21-23-25 mit distalem Anhänger an 25. Der Zahn 25 war endodontisch behandelt und zeigte im OPG eine deutliche apikale Aufhellung. Nach Aufklärung aller Therapiealternativen lehnte die Patientin eine prothetische Versorgung mit einem herausnehmbaren Zahnersatz ab. Es bestanden keine Vorerkrankungen, die eine Kontraindikation für den chirurgischen Eingriff darstellten. Neben dem OPG wurden für die präoperative Diagnosik Modelle zur Herstellung einer Bohrschablone angefertigt. Die Restknochenhöhe wurde anhand des OPGs an den verschiedenen geplanten Implantatpositionen ermittelt. Die präoperative Knochenhöhe betrug in Regio 024 8,8 mm, in Regio 026 7,2 mm und in Regio 027 8,0 mm. Aufgrund der unzureichenden Knochenhöhe mußte bei der Patientin eine Augmentation der Kieferhöhle vorgenommen werden, um eine erfolgreiche Implantation gewährleisten zu können.

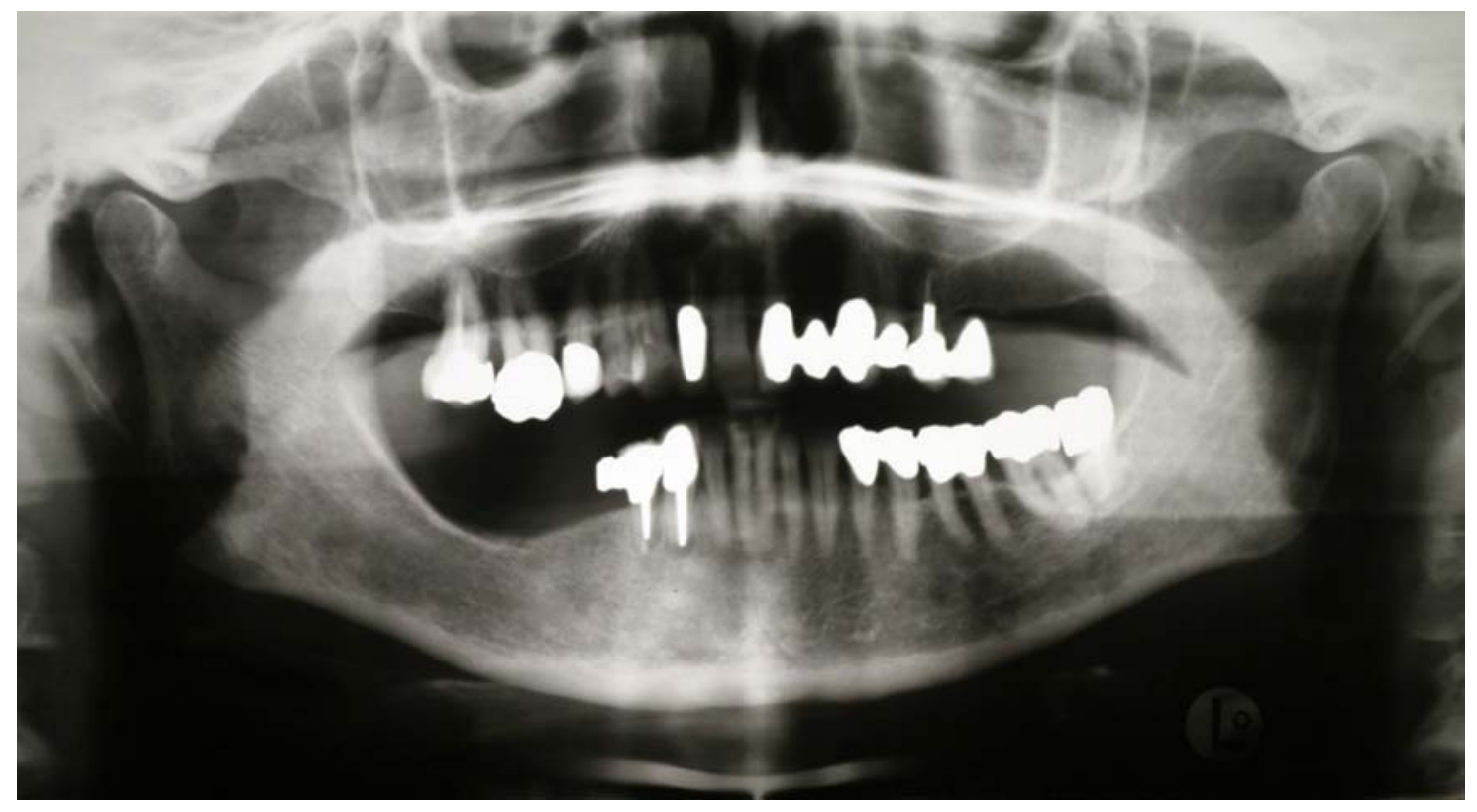

Abbildung 26: OPG präoperativ

Die Operation erfolgte mit der minimalinvasiven SALSA-Technik in einem einzeitigen Verfahren und der Sinus maxillaris wurde mit dem alloplastischen Knochenersatzmaterial Cerasorb (beta-TCP) gefüllt. Das gewählte Implantatsystem war das Xive-System. Bei allen Implantaten betrug die Länge $13 \mathrm{~mm}$. Der Durchmesser lag bei den Implantaten in Regio 024 und 027 bei 3,8 $\mathrm{mm}$ und beim Implantat in Regio 026 bei 3,4 $\mathrm{mm}$. Die postoperativ erreichte vertikale Augmentathöhe betrug für die Region 024 und 027 jeweils 6,4 mm und für die Region $0268,0 \mathrm{~mm}$. Es kam zu keinen intra - oder postoperativen Komplikationen. 


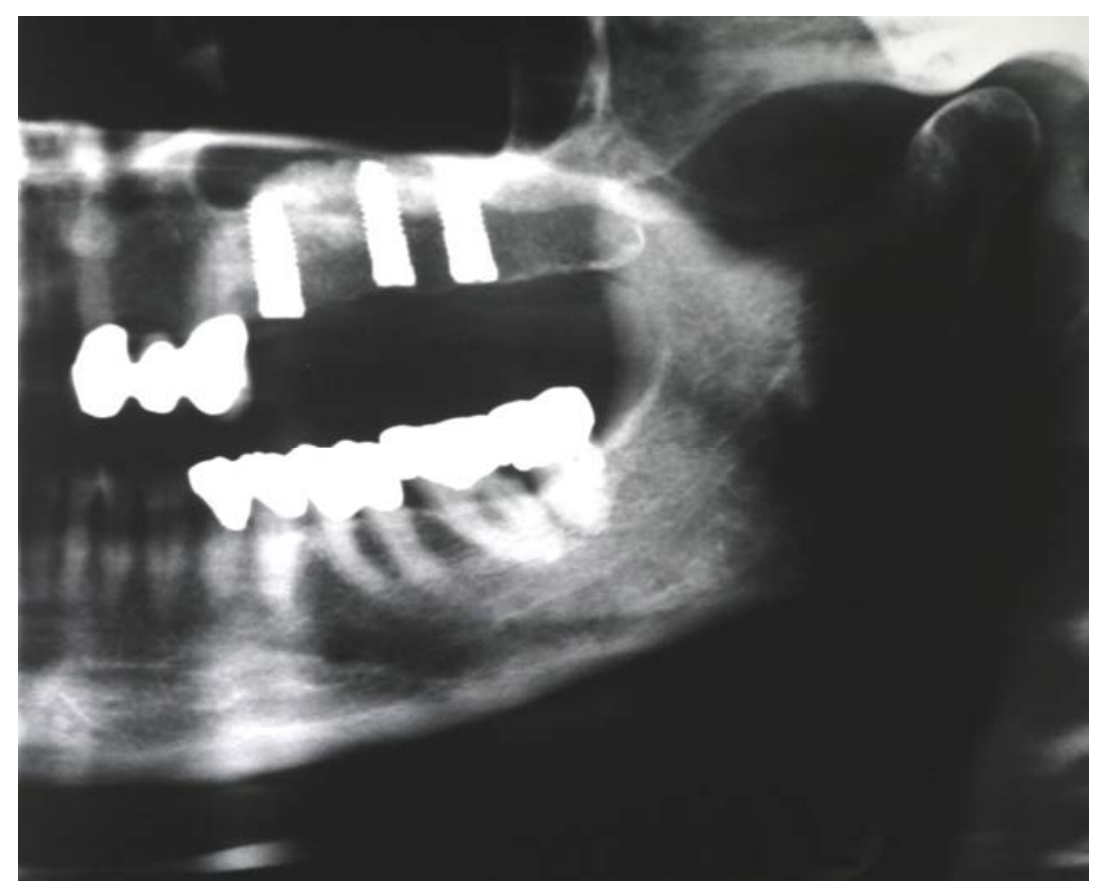

Abbildung 27: OPG postoperativ

Die Beobachtungszeit betrug bei der Nachuntersuchung 36 Monate. Die prothetische Suprakonstruktion war in situ und bestand aus einer implantatgetragenen Brücke. Der SBI betrug bei der Patientin 10\%. Die Sondierungstiefen wurden ermittelt und betrugen bei Implantat 024 mesial und distal jeweils $2 \mathrm{~mm}$, in Regio 026 mesial und distal jeweils $3 \mathrm{~mm}$ und in Regio 027 mesial $3 \mathrm{~mm}$ und distal $4 \mathrm{~mm}$. Es konnte kein Bluten auf Sondieren festgestellt werden. Da die Suprakonstruktion für die Kontrolluntersuchung nicht abgeschraubt werden sollte, ist es nicht möglich einen genauen Wert für jedes einzelne Implantat zu ermitteln. Der Periotestwert für die Implantate betrug -2. Im Rahmen der Nachuntersuchung wurde zur Verlaufskontrolle eine routinemäßige OPG-Aufnahme angefertigt, die eine Beurteilung des periimplantären Knochens ermöglichte. Der Knochenabbau in Regio 024 lag bei 2,4 mm, in Regio 026 bei $1,8 \mathrm{~mm}$ und in Regio 027 bei $1,4 \mathrm{~mm}$. Die Patientin war mit dem Gesamtbehandlungsergebnis sehr zufrieden und würde diese Operationsmethode weiter empfehlen.

Mit Hilfe der minimalinvasiven Sinusbodenaugmentation ist es möglich, die unilaterale Freiendsituation mit einem festen implantatgetragenen, adäquaten Zahnersatz zu versorgen. 


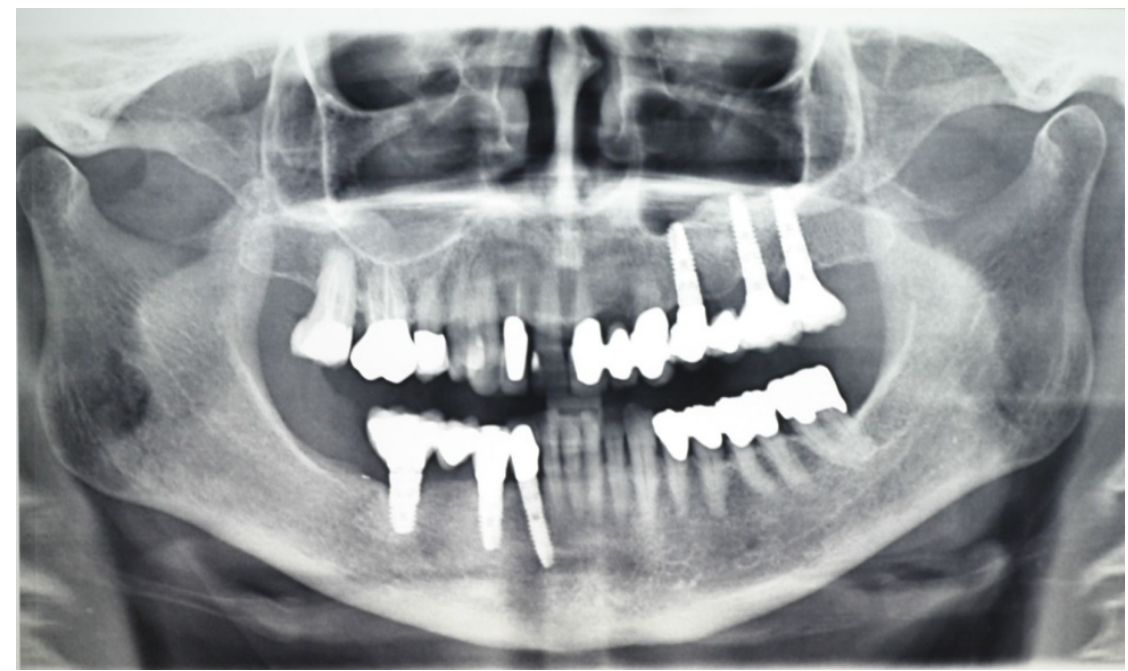

Abbildung 28: OPG vom Tag der Nachuntersuchung
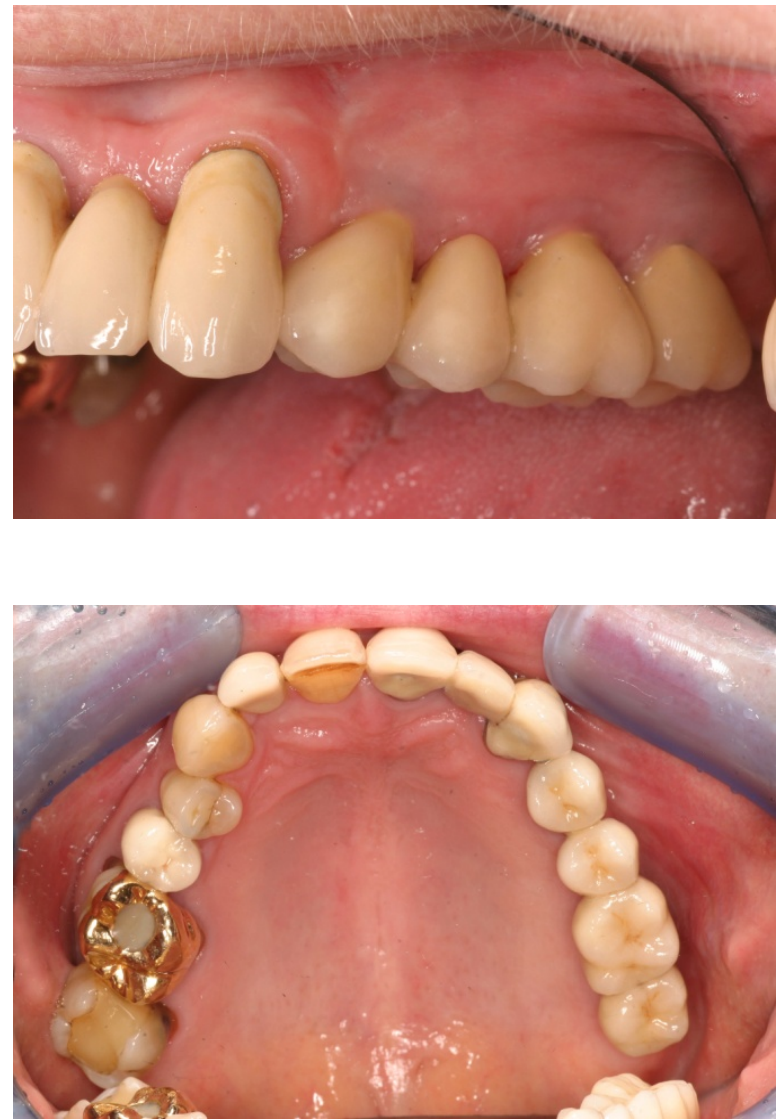

Abbildung 29 und 30: Fotos der Implantate mit Suprakonstruktion in situ 


\section{Diskussion}

\subsection{Methodenkritik: Radiologische Evaluation}

Die qualitative Aussagekraft von Röntgenbildern über die zirkuläre Knochenstruktur ist begrenzt, da dreidimensionale Objekte auf einen zweidimensionalen Film reduziert werden (Gómez-Román et al. 1995, 1999, Behneke A und Behneke N 2004). Jedoch wird dies toleriert, um den knöchernen Defekt bewerten zu können (Gómez-Román et al. 1999). Die verschiedenen Röntgenaufnahmen werden auf Grund der aufnahmetypischen Faktoren, wie die Zeichenschärfe des Films und die geräteabhängige Vergrößerung bei den Röntgenbildern (Szabo et al. 1991), uneinheitlich in der Literatur bewertet.

Um Knochenveränderungen einschätzen zu können empfehlen einige Autoren die Zahnfilmaufnahmen (Larheim und Eggen 1982, Cox und Pharoah 1986), während andere Autoren das Orthopantomogramm befürworten. Zudem ist diese Aufnahmetechnik leichter anzuwenden und die Strahlenbelastung für den Patienten fällt geringer aus (Behneke A et al. 1992, Gómez-Román et al. 1995). Das OPG bietet einen panorama-artigen Überblick über Ober- und Unterkiefer inklusive der Kiefergelenke. Allerdings ist das OPG auf Grund der linearen Verwischungen, Projektionen der Wirbelsäule und unterschiedlichen Verzerrungen der Bildregionen nur bedingt zur Verlaufskontrolle geeignet. Mit demselben Gerät und der weitestgehend gleichen Geräteeinstellung sind jedoch Dichte- und Volumenänderungen in der Implantatregion darstellbar.

Knochenresorptionen am Implantat können in den Röntgenbildern nur mesial und distal mit Messgenauigkeiten von $\pm 0,5 \mathrm{~mm}$ gemessen werden. Ursache hierfür sind die projektionsbedingten Überlagerungen (Jansen et al. 1993, Behneke A und Behneke N 2004).

Allerdings konnte in Untersuchen gezeigt werden, dass sich der tatsächliche Knochenabbau mit den röntgenologischen Messungen nur in ca. $60 \%$ aller Fälle mit einer Genauigkeit von $\pm 1 \mathrm{~mm}$ ermitteln lässt (Jansen et al. 1993, Eickholz und Hausmann 2000).

Albrektsson et al. (1986) postulierten die Auswertung von Röntgenbildern im Bereich von 0,2 mm. Die Durchführbarkeit dieser Forderung wurde allerdings bezweifelt (Jahn und d'Hoedt 1992, Jansen et al. 1993).

In der Reliabilitätsstudie von Gómez-Román et al. (1999) konnte eine 90\% Übereinstimmung der Strecken innerhalb eines Toleranzbereichs von $\pm 1 \mathrm{~mm}$ ermittelt werden.

Die Genauigkeit der Messungen an den Knochengrenzen ist von der periimplantären Transluzenz oder auch vom Implantat selbst abhängig. Die Dichteunterschiede von Knochen und Weichgewebe sind häufig im Kontrast nicht ausreichend, folglich kann hier die tatsächliche Knochenveränderung falsch eingeschätzt werden (Jansen et al. 1993, GómezRomán et al. 1995, 1999). 
Als Ursachen können zu geringe Knochenstärken, Entzündungsprozesse am Implantat, „Burnout"-Effekte, oder falsche Belichtungs- und Entwicklungszeit genannt werden (Gómez-Román et al. 1999).

Jansen et al. (1993) fordern Röntgenaufnahmen in regelmäßigen Intervallen mit annähernd gleich bleibenden Röntgeneinstellungen, um einen Knochenverlust ermitteln zu können.

Die digitale Nachbearbeitung von Röntgenbildern am Computer ermöglicht eine bessere Kontrastgebung. Durch zusätzliche Zoomeinstellungen können auch Aussagen bei Röntgenbildern mit niedriger Projektionsqualität erfolgen (Behneke A und Behneke N 2004).

Die Darstellung der periimplantären Knochenverhältnisse im Röntgenbild wird von vielen Autoren als wichtiges Kriterium im Hinblick auf die Implantatprognose gewertet, insbesondere bezüglich des gemessenen marginalen Knochenabbaus.

Keller U führte daher 1995 eine Studie zur Überprüfung der Messgenauigkeit an Knochentaschen durch. Die Tiefe der Knochentaschen an Implantaten wurde von drei erfahrenen Auswertern in Orthopantomogrammen sowie in Zahnfilmaufnahmen gemessen. Es wurde eine Übereinstimmung in 76\% der Fälle erreicht. Aus diesem Grund sollte die klinische Untersuchung im Vordergrund stehen und die Röntgendiagnostik ergänzend herangezogen werden (Keller U 1995).

OPGs wurden auch in anderen Studien zur Beurteilung der Sinusbodenaugmentationen und Implantate angefertigt (Keller EE et al. 1994, Blomqvist et al. 1996, Kahnberg et al. 2001, Strietzel 2004).

Um präzisere Aussagen über den augmentierten Bereich oder über Volumenänderungen treffen zu können sowie zur Planung von Implantaten werden moderne digitale Bildgebungsverfahren wie die Computertomographie (CT) oder die digitale Volumentomographie (DVT) herangezogen (Fredholm et al. 1993, Peleg et al. 1999, Ozyuvaci et al. 2005).

Mit den Systemen zur computerunterstützten Planung können anhand von CT- oder DVTDatensätzen 3D Planungen erstellt werden. Weiterhin ist die computerunterstützte Herstellung von individuellen Bohrschablonen möglich, die intraoperativ die geplante Implantatposition, die Implantatachse und die Gingivadurchtrittsstelle übertragen.

Insbesondere bei reduziertem Knochenangebot, in räumlicher Nähe zum Mandibularkanal oder zur Kieferhöhle stellt die CT- oder DVT- Untersuchung ein wertvolles ergänzendes diagnostisches Hilfsmittel dar (Fredholm et al. 1993).

Durch die Anwendung einer digitalen Aufnahmetechnik und eines dreidimensionalen Planungsprogramms kann das Knochenangebot besser genutzt und noch bestehende Indikationsgrenzen erweitert sowie das operative Risiko reduziert werden.

Die inkonstante gerätetypische Vergrößerung oder Verzerrung von Objekten auf OPGs oder eine fehlerhafte Patientenpositionierung muss vor der Implantatinsertion berücksichtigt werden (Haßfeld et al. 1998). 
In der vorliegenden Studie wurden die im Rahmen der Routinediagnostik bei allen Patienten durchgeführten Röntgenaufnahmen zur Bewertung des Operationsergebnisses herangezogen. Die angewendete Aufnahmetechnik stellte das OPG dar. Die durch Artefakte hervorgerufene Spaltbildung mesial und distal der Implantate bei der digitalen Bildauswertung wurde berücksichtigt. Trotzdem bleibt es schwierig Ergebnisse anhand von zweidimensionalen Bildern zu beurteilen.

\subsection{Implantatüberlebensrate}

In den letzten 30 Jahren hat sich die Erfolgserwartung von Implantaten deutlich verändert (Knöfler et al. 2004). Mit Verbesserung der Methoden und Materialien konnten die Implantatüberlebensraten gesteigert werden und erreichen heute in der Sinusliftregion ca. 85 bis 98\% (Davarpanah et al. 2002, Mazor et al. 2004, Bornstein et al. 2008, Johansson et al. 2010, Tetsch et al. 2010).

Langzeiterfolge in der Literatur sind jedoch nur bedingt miteinander vergleichbar. Es gibt keine einheitlichen Auswahlkriterien, die einen implantologischen Erfolg definieren. Bei einigen Autoren wird nur der reine Implantatverlust in Überlebenszeitanalysen als Misserfolg angeführt (Adell et al. 1981, Naert et al. 1991). Für andere Autoren spielen ganz spezielle Parameter für die Klassifikation eines Implantaterfolges eine Rolle.

Umfangreiche Daten zum Implantaterfolg nach Sinusbodenelevation liefern die Literaturübersichtsarbeiten von Wallace und Froum (2003) sowie von Del Fabbro et al. (2004). In beiden Studien konnte eine ähnlich gute Überlebensrate im augmentierten Sinus (92,6\% bzw. 91,49\%) verglichen mit Implantaten im nicht augmentierten Oberkieferseitenzahnbereich (95,1\% bzw. 94,2\%) ermittelt werden.

Auch Jensen und Terheyden (2009) stellten im Rahmen einer umfangreichen Meta-Analyse von 108 Studien zum Implantatüberleben fest, dass die Ergebnisse nach Sinusbodenaugmentation nicht schlechter ausfielen, als die in der Literatur berichteten Implantatüberlebensraten bei nicht augmentiertem Kiefer.

Engelke et al. (2003) ermittelten nach 56 Monaten eine Überlebensrate der Implantate nach Sinuslift von 95,7\%. In der Studie von Ferrigno et al. (2006) konnte eine 12-JahreÜberlebensrate von 94,8\% und eine Erfolgsrate von 90,8\% festgestellt werden.

Ein ähnlich gutes Ergebnis erzielten Peleg et al. (2006) mit einer Überlebensrate von 97,9\% nach neun Jahren. Ewers (2005) dokumentierte 95,6\% nach 13 Jahren Beobachtungszeit. Bei Tetsch et al. (2010) wurde nach 15 Jahren eine Überlebensrate von 97,1\% festgestellt. 
In der vorliegenden Studie wurden die guten Langzeitergebnisse einer dentalen Implantation in Kombination mit einer Sinusbodenelevation erneut bestätigt. Die Überlebensrate zum mittleren Beobachtungszeitraum von sechs Jahren betrug 95,8\% für das Gesamtkollektiv der Implantate. Die Implantatverluste fanden in unseren Untersuchungen wie in früheren Studien beschrieben überwiegend innerhalb des ersten Jahres statt (Isaksson 1994, Blomqvist et al. 1998, Knöfler et al. 2004). Die Mehrzahl der Implantate ging während oder kurze Zeit nach der Einheilphase von drei bis sechs Monaten verloren.

Für die Erfolgsbewertung wurden zum einen die Kriterien nach Jahn und d'Hoedt (1992) herangezogen, wobei eine Erfolgsrate von 94,7\% ermittelt wurde. Weiterhin wurden auch die Kriterien von Naert et al. (1992) und Snauwaert et al. (2000) berücksichtigt. Hieraus ergaben sich $94,4 \%$.

Bedenkt man, dass das vorliegende Studiendesign mit teilweise sehr geringen präoperativen Ausgangsknochenhöhen und einer simultanen Implantatinsertion ein, von anderen Autoren eher als riskant eingestuftes Verfahren vorsah (Misch und Dietsh 1994, Olson et al. 2000), sind die Ergebnisse der vorliegenden Studie vielversprechend.

Diese Ergebnisse zeigen, dass dentale Implantate selbst in der schwierigen posterioren Oberkieferregion eine zuverlässige und auf lange Sicht stabile Art der prothetischen Rehabilitation bei Zahnverlust darstellen.

\subsection{Einfluss der präoperativen Knochenhöhe auf das Implantatüberleben}

Für eine erfolgreiche Osseointegration von dentalen Implantaten ist der vitale eigenständige Knochen die beste Grundlage. Die Ausgangsknochenhöhe ist entscheidend für die Wahl eines ein- oder zweizeitigen Operationsverfahrens. Einige Autoren empfehlen die simultane Implantation nach Sinusbodenelevation nur in Oberkieferbereichen mit ausreichend hoher Ausgangsknochenhöhe, wenn eine Primärstabilität sicher erzielbar ist (Rosen et al. 1999, Brägger et al. 2004, Ferrigno et al. 2006).

In der Literatur ist die Ausgangsknochenhöhe ein entscheidender Faktor für den implantologischen Langzeiterfolg beim einzeitigen Vorgehen (Rosen et al. 1999, Toffler 2004). So werden bei präoperativen Knochenhöhen von $4 \mathrm{~mm}$ und weniger verminderte Überlebensraten der Implantate bei einzeitigem Vorgehen angegeben.

Kirsch et al. (1999) sehen die Primärstabilität der Implantate als entscheidenden Faktor für Erfolg und Misserfolg an. Kann diese beim einzeitigen Verfahren gewährt werden, so kann eine simultane Implantation erfolgen.

Der untere Grenzwert für die simultane Implantation wurde in der Literatur in den letzten Jahren stets weiter nach unten korrigiert. 
Peleg et al. (1998) führten erfolgreiche Sinusbodenaugmentationen mit simultaner Implantation bei präoperativen Knochenhöhen von nur 1 bis $2 \mathrm{~mm}$ durch. Sie erzielten eine Überlebensrate von $100 \%$ nach 39 Monaten Beobachtungszeit.

Toffler ermittelte in einer Studie von 2004 eine Überlebensrate von 93,5\% für alle untersuchten Implantate nach Sinusbodenaugmentation. Die Überlebensrate der einzeitig inserierten Implantate bei einer Restknochenhöhe von $4 \mathrm{~mm}$, betrug allerdings nur noch 73,4\%.

Auch andere Autoren berichteten über ähnliche Ergebnisse (Hirsch und Ericsson 1991, van den Bergh et al. 1998). Ein Zusammenhang zwischen Ausgangsknochenhöhe und Implantatverlust konnte allerdings nicht ausreichend belegt werden (Jensen et al. 1998, Wallace und Froum 2003, Stricker et al. 2003).

Tawil und Mawla (2001) konnten aus ihren Ergebnissen zur Sinusbodenelevation ableiten, dass die Überlebensrate von Implantaten sinkt, die in präoperative Knochenhöhen von weniger als 5 $\mathrm{mm}$ inseriert wurden.

Pjetursson et al. (2009) bestätigten dies in ihrer Studie. Die kumulative Überlebensrate nach einer mittleren Beobachtungszeit von 3,2 Jahren betrug 97,4\%. In Bezug auf die Ausgangssituation konnte eine Überlebensrate von 91,3\% bei einer Restknochenhöhe unter 4 $\mathrm{mm}, 90 \%$ bei einer Restknochenhöhe zwischen 4 und $5 \mathrm{~mm}$ und $100 \%$ bei mehr als $5 \mathrm{~mm}$ festgestellt werden.

Auch Mardinger et al. (2007) beschrieben eine bessere Erfolgsrate bei Ausgangsknochenhöhen über 4 mm (98,7\%) gegenüber Knochenhöhen zwischen 1 bis 3 mm (92\%).

Peleg et al. (2006) ermittelten ebenfalls eine bessere Überlebensrate für Implantate, die in mehr als 5 mm Knochen platziert wurden (98,9\%). Für Implantate in einer Restknochenhöhe von 1-2 $\mathrm{mm}$ betrug sie nach einem Beobachtungszeitraum von neun Jahren 94,6\%.

In der vorliegenden Studie wurde eine signifikant höhere Verlustrate für Implantate bei geringen Ausgangsknochenhöhen festgestellt $(\mathrm{p}=0,02600)$. Für die Ausgangsknochenhöhen unter 7,1 mm konnte eine Überlebensrate von 93,6\% bei einem mittleren Beobachtungszeitraum von 78 Monaten ermittelt werden. Für höhere präoperative Knochenhöhen wurde eine deutlich bessere Überlebensrate von 98,2\% festgestellt.

Damit werden die Ergebnisse anderer Autoren hinsichtlich einer verbesserten Überlebensrate bei höheren Ausgangsknochenhöhen bestätigt. 


\subsection{Einfluss der Implantatlokalisation auf das Implantatüberleben}

Die Implantatposition wird im Allgemeinen nach prothetischen Gesichtspunkten, also im Hinblick auf die spätere Versorgung, gewählt. Allerdings können die anatomischen Verhältnisse in Bezug auf die ortsständige Knochentopographie und Knochenqualität oft die gewünschte Positionierung hinsichtlich der Primärstabilität erschweren. Eine Verbesserung der Implantationsbedingungen ist daher anzustreben.

Die Bedeutung der Implantatlokalisation für das Implantatüberleben wird in der Literatur kontrovers diskutiert. Einige Autoren konnten in ihren Studien keinen Einfluss der Implantatlokalisation in Ober- oder Unterkiefer nachweisen (Ivanoff et al. 1999, Naert et al. 2002, Perry und Lenchewski 2004, Friberg et al. 2005, Gentile et al. 2005, Lemmerman KJ und Lemmerman NE 2005).

Ein signifikant höherer Implantatverlust generell im Oberkiefer wurde allerdings von anderen Autoren beschrieben (Eckert et al. 2001, Chuang et al. 2002, Strietzel et al. 2004, Moy et al. 2005, Mundt et al. 2006).

In der Untersuchung von Scurria et al. (1998) wurde eine tendenziell höhere Verlustrate für die in der posterioren Oberkieferregion inserierten Implantate im Vergleich zur Frontzahnregion ermittelt. Auch Shatkin et al. (2007) wiesen in der posterioren Maxillaregion die höchste Verlustrate nach.

Ebenso gaben McDermott et al. (2006) tendenziell bessere Überlebensraten für den anterioren Bereich an. Diese Tatsache könnte mit den geringeren Ausgangsknochenhöhen oder Knochenqualitäten im posterioren Bereich zusammen hängen (Ulm et al. 1999).

Im Gegensatz hierzu wurden durch andere Autoren bessere Überlebensraten in der posterioren Maxilla beschrieben (Fugazzotto et al. 1993, Nevins und Langer 1993, Haas et al. 1996).

Diese unterschiedlichen Ergebnisse können durch die lokalen anatomischen Verhältnisse bedingt sein. Lindh et al. (1998) sehen die Patientenselektion für die Implantatbehandlung als wesentlichen Einflussfaktor auf die Überlebensrate.

In der vorliegenden Studie traten sechs von 13 Implantatverlusten in der Zahnregion des ersten Molaren auf (Überlebensrate 92,6\%). Als mögliche Ursachen für die höhere Verlustrate in dieser Region kommen zum einen die höhere Kaubelastung für die im Seitenzahnbereich inserierten Implantate, die Knochenstruktur sowie die häufig geringste präoperative Knochenhöhe in dieser Region in Betracht.

Eine ausreichende Primärstabilität zu erzielen ist hier deutlich erschwert, und resultiert in einer höheren Misserfolgsrate (Friberg et al. 1991, Jaffin und Bermann 1991, Bain und Moy 1993).

In der Region des ersten Molaren wurde für die Kieferhöhle die basalste Ausdehnung gemessen. Das präoperative Knochenangebot in der vorliegenden Studie lag in dieser Zahnregion im Durchschnitt bei 5,1 mm. Der erste Molar steht im Kauzentrum und somit ist die okklusale Belastung während der Mastikation hier am Größten. 
Diese Vermutung wird durch das Betrachten der benachbarten Zahnregionen noch verstärkt. Die stabilste Region hinsichtlich des Implantatüberlebens waren die Prämolarenregionen. In der ersten Prämolarenregion gingen 2 Implantate verloren (Überlebensrate 96,9\%), obwohl in dieser Region nahezu die gleiche Anzahl an Implantaten inseriert wurde. Das durchschnittliche präoperative Knochenangebot in der ersten Prämolarenregion betrug 9,1 $\mathrm{mm}$ und stellte damit eine deutlich bessere Ausgangsknochenhöhe dar, als in der Region des ersten Molaren.

Jedoch ist die Datenlage nicht ausreichend, um verlässliche Aussagen über direkte Zusammenhänge zwischen Implantatinsertionsstellen, präoperativen Knochenhöhen und Implantatverlusten machen zu können.

\subsection{Einfluss der Sinusmukoperiostperforation auf das Implantatüberleben}

Die Perforation der Schneiderschen Membran ist die weitaus am häufigsten beschriebene Problematik bei Sinusliftoperationen (Chanavaz 1990, Timmenga et al. 1997, Kübler et al. 1999, Shlomi et al. 2004).

In der Studie von Proussaefs et al. (2004) wurden signifikante Ergebnisse ermittelt. Nach dem Verschluss von SMP-Perforationen mittels resorbierbarer Kollagenmembranen bei zweizeitigen Sinusliftoperationen, kam es zu einer signifikant niedrigeren Knochenneubildung als bei Sinusliftoperationen mit intakt gebliebenem SMP. Die Überlebensrate der Implantate war mit 54,5\% deutlich geringer bei perforierten SMP im Vergleich zu 100\% Überlebensrate, wenn keine Perforation auftrat.

Schwartz-Arad et al. (2004) fanden einen deutlichen Zusammenhang zwischen Perforationen des SMP und postoperativen Komplikationen, jedoch wurden keine erhöhten Implantatverluste bei perforierten SMP festgestellt. Dies wurde ebenfalls von Raghoebar et al. (2001) bestätigt. In der Studie von Shlomi et al. (2004) wurden zwei Patientengruppen röntgenologisch und klinisch untersucht, ob unterschiedliche Implantatüberlebensraten resultieren, wenn es intraoperativ zu Perforationen kam, die mit einer resorbierbaren Membran gedeckt wurden oder wenn während der Sinuliftoperation keine Perforation entdeckt wurde. In der ersten Gruppe wurde ein Implantatüberleben von 90\% und in der zweiten Gruppe, in der es intraoperativ zu keiner Perforation der Schneiderschen Membran kam, von 91\% festgestellt.

In der vorliegenden Studie traten bei insgesamt 133 operierten Kieferhöhlen 25 Perforationen auf (Perforationsrate 18,8\%). Beim einzeitigen Verfahren wurden bei 15 Patienten Perforationen und beim zweizeitigen Verfahren bei drei Patienten Perforationen festgestellt.

Bei 11 Patienten waren die Perforationen einseitig und bei sieben Patienten beidseitig entdeckt worden. Von insgesamt 13 verloren gegangenen Implantaten waren in vier Fällen Perforationen des SMP vorhanden. 
In 4 von diesen 25 Kieferhöhlen mit Membranperforation (16,0\%) ging eine Anzahl von $n=4$ Implantaten verloren. Demgegenüber steht die Anzahl von 108 Kieferhöhlen, bei deren Operation es nicht zu einem Einreißen der Membran kam. Bei sieben dieser insgesamt 108 intakten Kieferhöhlen (6,5\%) kam es zu einem Implantatverlust von insgesamt $\mathrm{n}=9$ Implantaten, wobei insgesamt drei Implantate bei einem Patienten verloren gingen.

Verschiedene Autoren vermuten (Vlassis und Fugazzatto 1999, van den Bergh et al. 2000), dass nach einer Perforation der Schneiderschen Membran das regenerative Potential verschlechtert ist und fordern, dass nach Membranperforationen keine simultane Implantation stattfinden sollte (Shlomi et al. 2004).

Mit einer Häufigkeit von 18,8\% traten in der vorliegenden Studie vergleichsweise weniger Perforationen beim Sinuslift auf als in der Literatur beschrieben (Block und Kent 1997: 20 \%, Wilkert-Walter et al. 2002: $25 \%$, Stricker et al. 2003: 37,87\%, Shlomi et al. 2004: $28 \%$, Schwartz-Arad et al. 2004: $44 \%$, Becker et al. 2008: 20,4\%).

Gründe hierfür können einerseits die chirurgischen Erfahrungen des Operateurs darstellen, andererseits wird mit Hilfe der Endoskopie im Rahmen der SALSA das Übersehen einer Schleimhautperforation reduziert.

Die hohe Implantatüberlebensrate in der vorliegenden Studie ist vergleichbar mit den Ergebnissen anderer Autoren, die keinen signifikanten Einfluss von intraoperativen Komplikationen auf das Implantatüberleben feststellen konnten (Ardekian et al. 2006, Barone et al. 2006).

Bei entsprechender Versorgung einer intraoperativ aufgetretenen Perforation der Kieferhöhlenschleimhaut ist kein negativer Einfluss auf das Implantatüberleben zu befürchten. Für einen von anderen Autoren geforderten Abbruch der Operation bei einem solchen Ereignis gibt es also keine Grundlage (Khoury et al. 1993, Fugazzotto und Vlassis 2003). 


\subsection{Einfluss des Augmentats auf das Implantatüberleben}

Viele Autoren befürworten als suffizientes Knochenersatzmaterial vor allem autogenen Knochen oder ein Gemisch aus autogenem Knochen und Knochenersatzmaterialien (Hallman et al. 2002, Wallace und Froum 2003, Zijderveld et al. 2005).

In der Literatur stellt Cerasorb (ß-TCP) das mit Abstand am häufigsten verwendete und erfolgversprechendste Regenerationsmaterial dar (Nkenke et al. 2002, Merkx et al. 2003, Szabo et al. 2005, Zijderveld et al. 2005).

Szabo et al. (2005) führten eine intra-individuelle Vergleichstudie bei 20 Patienten mit Sinusbodenaugmentation durch unter Verwendung von Cerasorb im Vergleich $\mathrm{zu}$ autogenem Knochen. Es wurde festgestellt, dass ß-Tricalziumphosphate ein zufriedenstellendes Augmentationsmaterial sind, auch ohne Zusatz von autologen Knochen.

In der Studie von Reinhardt und Kreusser (2000) wurde Cerasorb als alleiniges ATM in der Sinusliftregion verwendet. Bei 39 Patienten wurde ein Sinuslift vorgenommen und insgesamt 101 Implantate inseriert. Die Implantatüberlebensrate für den Beobachtungszeitraum von 3,5 Jahren betrug 99\%.

Engelke et al. (2003) konnten ähnlich gute Ergebnisse ermitteln. Bei 83 Patienten wurde entweder ein - oder beidseitig eine Sinusbodenaugmentation durchgeführt und simultan insgesamt 211 Implantate positioniert. Als ATM wurden unterschiedliche Mischungen aus Cerasorb und autogenem Knochen eingesetzt. Die erzielte Implantatüberlebensrate nach fünf Jahren betrug $94,79 \%$.

Interessante Ergebnisse lieferte die Vergleichsstudie zu Augmentationsmaterialien von Del Fabbro et al. (2004). Es wurde eine Überlebensrate von 87,70\% für rein autogenen Knochen, von 94,88\% für eine Kombination aus autogenem Knochen und Knochenersatzmaterial (KEM) und sogar 95,98\% bei der alleinigen Verwendung von KEM festgestellt.

Esposito et al. (2006) propagieren bei Sinusliftoperationen mit einer Restknochenhöhe unter 5 mm die Verwendung von Knochenersatzmaterialien statt autologem Knochen.

Jensen et al. (1998) konnten unter Zugrundelegung der in der Konsensuskonferenz in Boston zusammengetragenen Daten von insgesamt 2997 Implantaten in 1007 Sinuslift-Operationen keinen signifikanten Unterschied im Implantatüberleben in den einzelnen Materialien feststellen.

In der vorliegenden Studie konnten für die verwendeten Augmentationsmaterialien, autogener Knochen und alloplastische Materialien (Cerasorb, Bioresorb und AlgiPore), unterschiedliche Überlebensraten der Implantate ermittelt werden.

Für den autologen Knochen ergaben sich 90,1\% (n=54) und für alloplastisches Material 97,4\% $(\mathrm{n}=266)$ nach der mittleren Beobachtungszeit von 78 Monaten. Die hier vorliegenden Ergebnisse wiesen eine Signifikanz auf $(\mathrm{p}=0,0066)$. 
Damit ergibt sich ein deutlich besseres Ergebnis für alloplastische Materialien im Vergleich zu der alleinigen Verwendung des als Goldstandard propagierten autologen Knochens.

Allerdings ist hierzu lediglich eine Aussage über eine Tendenz möglich. Die generelle Betrachtungsweise ist streng genommen nicht exakt, da die Gruppen mit den verschiedenen Augmentationsmaterialien nicht homogen waren.

Trotz der guten Ergebnisse von Knochenersatzmaterialien wird heute noch autogener Knochen als ATM bei der Sinusbodenaugmentation verwendet. Einige Autoren sehen in der osteogenen Potenz des autogenen Knochens die Fähigkeit ein qualitativ besseres Implantatlager zu generieren (Moy et al. 1993, Buser et al. 1998).

Allerdings kann die Notwendigkeit einer autogenen Knochenentnahme im Rahmen eines Sinusliftes nach den vorliegenden Ergebnissen in der Literatur in Frage gestellt werden. Bei gleich guten oder sogar besseren Therapieresultaten mit KEM kann den Patienten eine zusätzliche Entnahmemorbidität durch Schmerzen, Narben, Bewegungseinschränkung und Klinikaufenthalt erspart werden (Del Fabbro et al. 2004).

Auch Wallace und Froum (2003) und Buser et al. (2002) stellten klar heraus, dass aus der Verwendung von autogenem Material zur Sinusbodenaugmentation keine Verbesserung der Implantatüberlebensrate resultiert. Dies kann durch unsere Ergebnisse bestätigt werden.

\subsection{Mundhygiene und periimplantäre Gesundheit}

Der Zusammenhang zwischen der Kontamination der Implantatoberfläche mit bakterieller Plaque und periimplantärer Erkrankung wurde eingehend in der Literatur dargestellt (Lindhe et al. 1992, Lindquist et al. 1996, Esposito et al. 1998).

Die Mundhygiene hat einen entscheidenden Einfluss auf die Langzeitprognose dentaler Implantate (Heitz-Mayfield 2008, Lindhe und Meyle 2008). Verschiedene Faktoren wie das Alter der Patienten, deren manuelle Geschicklichkeit, Position und Anzahl der Implantate sowie die Gestaltung der prothetischen Suprastruktur wirken sich auf die individuelle Mundhygienefähigkeit aus.

Nach der Ansicht von Behneke A und Behneke N (2004) werden deshalb zum langfristigen Erhalt des Therapieerfolges die regelmäßige Überwachung und gegebenenfalls begleitenden Therapiemaßnahmen für zwingend notwendig angesehen.

Kommt es aufgrund mangelnder Pflege zur Plaqueretention an Implantaten, reagiert das periimplantäre Weichgewebe mit Entzündungsreaktionen. 
Folgende klinische Symptome können auftreten (Mombelli \& Lang 1998):

- Radiologisch erkennbarer periimplantärer Knochenverlust

- Periimplantäre Zahnfleischtaschen

- Schwellung der periimplantären Weichgewebe.

Schreitet die entzündliche Erkrankung fort, entsteht das Krankheitsbild der Periimplantitis. Diese ist definiert als „ein entzündlicher Prozess, der das periimplantäre Gewebe von osseointegrierten und in Funktion stehenden Implantaten betrifft und in einem Verlust des umgebenden Knochens resultiert“ (Mombelli und Lang 1998, S.63).

Die Messung der Taschentiefe ist eine wichtige Methode, um mögliche resorptive Knochenveränderungen einschätzen zu können. Die Sondiertiefenmessung alleine erlaubt aber keine aussagekräftigen Rückschlüsse über die periimplantäre Situation.

Allerdings ist die Taschenmessung dennoch geeignet zur Vergleichbarkeit bei Untersuchungen sowie im Anschluss an eine Periimplantitistherapie, um den langfristigen Erfolg bewerten zu können.

Veränderungen der Sondierungstiefe sind besonders aussagekräftig bei der Früherkennung der Periimplantitis (Rüdiger und Flemming 1995). Zumal durch die radiologische Kontrolle nur der mesiale und distale Knochen beurteilt werden kann und mit Hilfe der Sondierungstiefe das zirkuläre Knochenniveau bewertet wird. Es ist daher immer ratsam eine Taschenmessung mit einer radiologischen Kontrolle zu kombinieren.

Festgestellte Blutungen müssen aber nicht immer Ausdruck einer periimplantären Entzündung sein, sondern sind mitunter als Verletzungsfolge durch einen zu hohen Sondierungsdruck zu verstehen (Lekholm 1986).

Aufgrund des Zusammenhangs von Mundhygiene und der Entstehung von periimplantären Erkrankungen ist es dennoch wichtig diese Parameter regelmäßig zu erheben, um periimplantäre Veränderungen frühzeitig zu erkennen und dadurch einen Implantatmisserfolg evtl. zu vermeiden (Pontoriero et al. 1994).

Im Patientengut dieser Studie konnte an $n=51$ Implantaten eine periimplantäre Entzündungsreaktion in Form von Blutung auf Sondierung nachgewiesen werden. Dies entspricht einem Anteil von 15,9\% aller untersuchten Implantate. Roos-Jansaker et al. konnten in ihrer Studie von 2006 eine ähnliche Verteilung feststellen.

Von allen Implantaten $(n=307)$, die im Rahmen dieser Studie nachuntersucht worden waren, konnte bei zwei Patienten an n=2 Implantaten eine Periimplantitis nachgewiesen werden.

Diese Implantate zeigten erhöhte Sondierungstiefen $(>5 \mathrm{~mm})$ und größere periimplantäre Knochenabbauraten. Bei Taschensondierung resultierte eine starke Blutung, jedoch ohne putride Sekretion. 
Die durchschnittliche Sondierungstiefe in der vorliegenden Studie betrug 2,9 mm mesial und 3,3 $\mathrm{mm}$ distal. Diese Werte sind durchaus als physiologisch zu werten. Es handelte sich um aufgeklärte und motivierte Personen bezüglich der Mundhygiene, was durch die mehrheitlich unauffälligen Gingivabefunde an den Implantaten bestätigt wurde.

Pjetursson et al. (2009) und Ferrigno et al. (2006) ermittelten vergleichbare Sondierungstiefen. Der überwiegende Anteil (67,0 \%) der untersuchten Implantate wies nur sehr geringe Taschentiefen $(<4 \mathrm{~mm})$ auf, was für einen guten periimplantären Gewebezustand spricht, wenn man von den in der Literatur beschriebenen Idealwerten von 1,5 bis 3,5 $\mathrm{mm}$ ausgeht.

Mitunter werden aber auch höhere Sondierungstiefen, abhängig vom Schleimhauttyp, von einigen Autoren als physiologisch beschrieben (Behneke A et al. 1997).

Es konnte, wie auch bereits in anderen Studien (Quirynen and Listgarten 1990, Brägger et al. 1996) beobachtet, eine Verbindung zwischen Taschentiefe und Knochenabbau gefunden werden.

\subsection{Implantatstabilität}

Mit Hilfe der Periotestmessungen können Knochenumbauvorgänge beurteilt werden, da sich eine $\mathrm{Zu}$ - oder Abnahme der Intensität des Knochen-Implantat-Interfaces an den Werten ablesen lässt (d'Hoedt und Schramm-Scherer 1988, Kovács 1998). Außerdem können osseointegrierte Implantate von bindegewebig eingeheilten enossalen Pfeilern objektiv unterschieden werden (d'Hoedt und Schramm-Scherer 1988).

Die jeweiligen verwendeten prothetischen Versorgungen beeinflussen jedoch die Periotestwerte. So können Knopfanker ankylotische und implantatgetragene Brücken zahnähnliche Periotestwerte verursachen (Kovács 1998).

Brägger et al. (1996) folgerten aus ihren Daten, dass Periotest-Messungen keine verläßliche Prognose zuließen. Die Kombination von Sondierung und radiologischen Parametern hingegen erlaubte eine verlässliche Einschätzung des periimplantären Status.

Hanisch et al. (1997) berichteten über Periotestwerte zwischen $-0,4+/-1,8$ bei Implantaten in der augmentierten Kieferhöhle. Olson et al. (1997) gaben durchschnittliche Periotestwerte von $-3,36$ an. Bornstein et al. (2008) ermittelten einen durchschnittlichen Periotestwert von -3,0. Somit kann davon ausgegangen werden, dass ein Implantat mit einem Periotestwert von -2,42, entsprechend dem Mittelwert, der in der vorliegenden Studie gemessenen Periotestwerte, als fest und osseointegriert angenommen werden kann. 


\subsection{Marginaler Knochenabbau}

Die regelmäßige Erhebung des knöchernen Attachmentlevels gilt als ein wichtiger Parameter zur Früherkennung pathologischer Veränderungen im periimplantären Gewebe, ebenso wie im Parodont natürlicher Zähne (Mombelli und Lang 1994).

Strid (1985) empfiehlt im Rahmen der periimplantären Diagnostik jeweils sechs und zwölf Monate nach Eingliederung der Aufbauten und dann einmal pro Jahr Röntgenbilder zu erstellen. Nach Brägger (1994) sollen direkt im Anschluss an die Operation Kontrollaufnahmen angefertigt werden, um früh einen Referenzpunkt für spätere Vergleiche festzuhalten.

Schon Adell et al. (1981) und Albrektsson et al. (1986) zogen für die Ermittlung eines implantologischen Langzeiterfolges den vertikalen Knochenverlust an Implantaten als Erfolgskriterium heran. Adell et al. (1981) formulierten einen Knochenabbau von 1,2 mm im ersten Jahr und im weiteren Verlauf $0,1 \mathrm{~mm}$ jährlich.

Spiekermann et al. (1995) sahen eine Knochenresorption von $<4 \mathrm{~mm}$ als Erfolgskriterium, andere Autoren gaben einen prozentualen Anteil des konstruktiv enossalen Implantatabschnittes an (Jahn und d'Hoedt 1992).

In zahlreichen Studien wurde die Problematik des periimplantären Knochenabbaus genau untersucht. In der Studie von Adell et al. (1990) zum einzeitigen Sinuslift beträgt der Knochenabbau 1,49 $\mathrm{mm}$ nach einem Jahr funktioneller Belastung, während die weitere Abbaurate pro Jahr 0,1 mm beträgt.

Auch Keller (1995) konnte sehr häufig einen postoperativen Knochenabbau feststellen, der zum Teil von anderen Autoren als ,physiologisch“ bezeichnet wurde (Schramm-Scherer et al. 1989, Keller 1995), also keine pathologische Veränderung darstellt.

Engelke et al. (2003) stellten einen mittleren Knochenverlust nach einem Jahr von 0,7 mm mesial und $0,6 \mathrm{~mm}$ distal fest.

In den Studien von Simion et al. (2004) und Jovanovic et al. (1992) wurde ein Knochenabbau jeweils von 1,7 mm über einen Zeitraum von 1-7 Jahren angegeben.

Ein ähnliches Ergebnis wurde in der Studie von Kahnberg und Vannas-Löfqvist (2008), mit einem marginalen Knochenabbau von 1,6 mm nach 5 Jahren, ermittelt.

Besonders während der Einheilungsphase und im ersten Jahr der funktionellen Belastung durch den Zahnersatz sind bei jedem osseointegrierten Implantat erhöhte Knochenabbauraten zu verzeichnen (Cox und Zarb 1987, Buser et al. 1990).

Adell et al. (1981) ermittelten in ihrer Studie, dass der hohe initiale Knochenverlust durch Abhebung des Periosts, chirurgisches Trauma (Entfernung von Knochen) und Spannungskonzentration durch Applikation der Suprakonstruktionen bedingt sei. 
Kitamura et al. (2004) konnten in ihrer Studie ermitteln, dass die Ursache des vermehrten Knochenabbaus, der innerhalb der ersten Belastungsphase auftritt, in der Einleitung von Kräften in den Knochen und den daraus resultierenden adaptativen Vorgängen (Remodelling) zu finden ist. Die Resorption des marginalen Knochenanteils unter Belastungseinfluss stellt somit eine physiologische Adaptation des Knochenlagers an die Belastungssituation dar. Jedoch ist auch nach Abschluss dieser ersten physiologischen Reaktion die Höhe des periimplantären Knochens keine konstante Größe, sondern wird zeitlebens Umbauvorgängen unterworfen.

Schlegel et al. (2007) ermittelten im Seitenzahngebiet einen durchschnittlichen Verlust der ursprünglichen Augmentationshöhe von 38,72\% ( = 4,6 mm) nach fünf Jahren. Der vertikale Höhenverlust im Frontzahngebiet hingegen belief sich auf 28,26\% (2,2 mm).

Es ergab sich über unseren Untersuchungszeitraum ein durchschnittlicher Knochenabbau von 2,9 mm. Für das einzeitige Operationsverfahren wurde ein mittlerer Knochenabbau von 2,8 mm, für das zweizeitige Verfahren von 3,3 mm ermittelt. Diese Werte des durchschnittlichen Knochenabbaus bei unseren Patienten sind vergleichbar mit den Werten in der Literatur (Nevins et al. 1998, Weber et al. 2000, Simion et al. 2001).

Unserer Ergebnisse ergaben weiterhin, dass die Implantatposition einen signifikanten Einfluss auf den Knochenverlust hatte $(\mathrm{p}=0,0453)$. Der Knochenabbau nahm $\mathrm{zu}$, je weiter distal im Kiefer die Implantate inseriert wurden. Als mögliche Ursachen hierfür werden die Dichte und der Vaskularisationsgrad des Knochens diskutiert. Diese Faktoren haben Einfluss auf die Umbaurate und den Knochenabbau (Adell et al. 1981, Pham et al. 1994).

Nach Schlegel et al. (2007) könnte der Grund für den unterschiedlichen Ablauf des Knochenabbaus in der Adaptation des Augmentats an dem ortständigen Knochen im Sinne der Remodellierung liegen. 


\subsection{Vergleich von einzeitiger und zweizeitiger SALSA}

Je nach Ausmaß der Atrophie können Implantate im Zusammenhang mit der Sinusbodenelevation entweder gemeinsam mit der Augmentation (einzeitiges / simultanes Vorgehen) oder zu einem späteren Zeitpunkt (zweizeitiges Vorgehen) inseriert werden.

Viele Autoren sehen das zweizeitige Verfahren als weniger riskant an (Lekholm et al. 1999, Pikos 1999, Kahnberg et al. 2001). Da diese Methode jedoch erhöhte traumatische Belastung sowie Kosten einer zweiten Operation und verlängerte Behandlungszeiten bedeutet (Raghoebar et al. 2001), wurde das zweizeitige Vorgehen in der vorliegenden Studie nur bei Grenzindikationen von besonders geringem präoperativen Knochenangebot angewendet.

Bei besonders stark atrophierten Kiefern bzw. wenn keine sichere Primärstabilität und optimale Implantatposition erzielt werden konnte, erfolgte die Einheilung des Transplantats in Übereinstimmung mit der Literatur 3 bis 6 Monate am Boden der Kieferhöhle, bevor die Implantate inseriert wurden (Tatum et al. 1993, Lekholm et al. 1999, Wilkert-Walter et al. 2002, Szabo et al. 2005, Zijderveld et al. 2005).

Tatum et al. (1993) und Lekholm et al. (1999) befanden eine Restknochenhöhe von $5 \mathrm{~mm}$ als Grenzwert für eine simultane Implantation, während Peleg et al. bereits 1998 eine Restknochenhöhe von $3 \mathrm{~mm}$ als ausreichend ansahen.

Bei der einzeitigen Therapie muss eine ausreichende anfängliche Stabilität sowohl des Augmentates, als auch der Implantate gewährleistet sein (Blomqvist et al. 1997). Ist diese bei der Simultanimplantation zu erzielen, kann die einzeitige Vorgehensweise gewählt werden (Kahnberg et al. 2001, Huang et al. 2004, Mazor et al. 2004). Khoury et al. (1993) bevorzugen das simultane Vorgehen aufgrund der besseren Prognose.

Andere Autoren sind jedoch der Ansicht, dass bei einem zweizeitigen Operationsverfahren eine suffizientere Implantatintegration erzielt werden kann (Lekholm et al. 1999, Schliephake et al. 1999).

Nach Jensen (1994) liefert ein zweizeitiges, gestaffeltes Procedere bessere Ergebnisse bzw. nach Hürzeler (1997) wird ein höherer Anteil an Knochen-Implantat-Kontakten in der augmentierten Region erreicht.

Als Vorteil des einzeitigen Vorgehens ist allgemein die verkürzte Gesamtbehandlungszeit zu nennen, da eine weitere Operation zur Insertion der Implantate wegfällt. Nachteilig ist jedoch die erhöhte Gefahr einer prothetischen Fehlpositionierung. Für eine ausreichende Primärstabilität ist es mitunter nötig, die optimale Implantatposition geringfügig zu verlassen. Dies kann anschließend die prothetische Versorgung erschweren (Blomqvist et al. 1997).

Demgegenüber steht das zweizeitige Procedere, dessen Vorteile sich zunächst darin widerspiegeln, dass eine erneute Planung, und bei Bedarf sogar eine Nachaugmentation möglich ist. 
Nach Einheilung des Augmentates ist der Knochen vaskularisiert und durch den Umbau ergibt sich eine festere spongiöse Struktur, in die die Implantate mit einer erhöhten Primärstabilität sicherer platziert werden können (Blomqvist et al. 1997).

Der Nachteil des zweizeitigen Vorgehens liegt in der längeren Gesamtbehandlungsdauer. Die Dauer bis zur endgültigen prothetischen Rehabilitation kann bei sekundärer Implantation bis zu zwei Jahre dauern. Problematisch ist auch die fehlende Krafteinleitung in den Knochen während der Einheilzeit, mit dem konsekutiv verbundenen teilweisen Transplantatverlust durch Resorption, insbesondere wenn es sich ausschließlich um autologen Knochen handelt. Diesem Verlust kann man jedoch in gewisser Weise entgegenwirken, wenn man KEM zumischt, die nur langsam resorbiert werden (Martini 2001).

Wallace und Froum verglichen in einem „systematic review“ von 2003 acht randomisierte, klinische Untersuchungen zur Implantation nach Sinuslift. Der Erfolg für eine einzeitige Implantation lag bei 89,7\%, während die zweizeitige Implantation 89,6\% erreichte.

In der vorliegenden Studie konnten 295 Implantate in einem einzeitigen Operationsverfahren gesetzt werden. Die Überlebensrate betrug 96,2\%. Auf Grund eines ungenügenden Knochenangebotes und einer nicht zu erreichenden Primärstabilität wurden 25 Implantate in einem zweizeitigen Vorgehen inseriert. Bei diesem Procedere betrug die Überlebensrate 92,0\%.

Zusammenfassend kann festgestellt werden, dass auch bei geringen Ausgangsknochenhöhen simultan implantiert werden kann, sofern Primärstabilität erzielbar ist.

\subsection{Patientenzufriedenheit}

Bis zum jetzigen Zeitpunkt liegen noch keine einheitlichen Erfolgskriterien für die Implantologie vor. Jedoch konnte in Studien festgestellt werden, dass rein klinische Erfolge nicht unbedingt zufriedene Patienten bedeuteten. Die Beurteilung der Patienten korreliert oft nicht mit dem klinischen Erfolg einer Behandlung (Awad und Feine 1998).

Einige Autoren befürworten zusätzlich die subjektive Bewertung durch den Patienten für die Erfolgsbeurteilung (Walton und MacEntee 1994, Feine et al. 1994, Buch et al. 2002, 2003, Homann et al. 2004).

Meyle (1984) sieht die psychologische Akzeptanz einer Therapie durch den Patienten sogar als entscheidend für den Langzeiterfolg.

Awad et al. (2000) und Allen et al. (2001) verwendeten in ihren Studien für die Patientenbefragung das OHIP (Oral Health Impact Profile). Es sollte die Lebensqualität zahnloser Patienten vor und nach der Behandlung entweder mit implantatgetragenen Prothesen oder konventionellen Totalprothesen evaluiert werden. 
Es wurde dargestellt, dass die Lebensqualität in beiden Gruppen nach der Behandlung angestiegen war. Allerdings war der Gruppenunterschied signifikant zugunsten der Implantatversorgung.

Awad und Feine (1998) kamen zu vergleichbaren Ergebnissen. In einer randomisierten Studie wurden Totalprothesen und Implantat-Deckprothesen verglichen. Die Gesamtzufriedenheit wurde maßgeblich beeinflusst durch die Zufriedenheit mit dem Komfort, der Stabilität, dem Aussehen, der Kau- und Sprachfunktion sowie dem Geschlecht der Probanden.

Auch Meijer et al. (1999), Cibirka et al. (1997) und Heydecke et al. (2003) kamen ebenfalls zu dem Ergebnis der höheren Zufriedenheit bei Implantatprothesen.

Die überwiegend positive Einstellung gegenüber implantologischen Versorgungen zeigte sich auch in der Patientenzufriedenheitsanalyse von Buch et al. (2002).

Ebenso konnten auch Pröbster und Weber (1989) sowie Kaptein et al. (1998) eine enorme Zustimmung der Patienten zur Implantatbehandlung verzeichnen, die trotz hohen Aufwandes und Kosten deutlich geäußert wurde.

Die Auswertung der Patientenfragebögen der vorliegenden Studie zeigt, dass die Implantologie bei den Patienten sehr gut akzeptiert wird, und die Erwartungshaltung an eine bessere prothetische Versorgung erfüllt wurde. $65 \%$ der Patienten in der vorliegenden Studie beurteilten das Gesamtresultat als „sehr gut“, weitere 33\% bewerteten dieses mit ,gut“.

Wichtige Kriterien wie Halt und Kaufunktion des implantatgetragenen Zahnersatzes wurden jeweils von über 90\% der Patienten als ,gut“ oder ,sehr gut“ bewertet.

Für die Mehrzahl der Patienten stellte sich der Eingriff als relativ unbelastend heraus. 19\% der Patienten mussten aufgrund von Problemen oder Beschwerden 1-2 mal zusätzlich einen Zahnarzt aufsuchen.

Die Patienten stimmten überein, dass die Lebensqualität mit Hilfe des neuen Zahnersatzes gestiegen sei und sogar 90\% der Patienten würden die implantologische Behandlung weiter empfehlen.

Der Wunsch nach einem hochwertigen Zahnersatz scheint bei den meisten Patienten stärker zu sein als die Angst vor intra- oder postoperativen Komplikationen. Die oft bestätigten geringen postoperativen Beschwerden, sprechen für das Konzept der implantologischen Versorgung des Oberkieferseitenzahngebietes.

Abschließend kann festgestellt werden, dass die dentale Implantologie als voll integrierte zahnärztliche Therapiemaßnahme angesehen werden kann, sowohl aus Sicht des Behandlers als auch aus Sicht des Patienten. 


\section{Schlussfolgerung}

Aus dieser Nachuntersuchung können folgende Schlussfolgerungen hinsichtlich der Langzeitresultate gezogen werden:

- Die SALSA-Technik stellt als minimalinvasive Sinusbodenaugmentation mit einer ImplantatÜberlebensrate von 95,8\% nach einem mittleren Beobachtungszeitraum von 6 Jahren eine erfolgreiche ergänzende Behandlungsmethode zur Implantation dar.

- Auch bei geringer Knochenhöhe kann das Augmentationsverfahren mit Erfolg eingesetzt werden. So kann unter Verwendung der SALSA-Technik ein adäquates Implantatlager mit ausreichender vertikaler Höhe geschaffen werden.

- Bei entsprechender Mundhygiene der Patienten liegen physiologische Sondierungstiefen und gesunde periimplantäre Verhältnisse vor. Des Weiteren zeigen die ermittelten Periotestwerte feste und osseointegrierte Implantate an.

- Die auffallende Zustimmung der Patienten auf Grund geringer postoperativer Beschwerden und einer Steigerung ihrer Lebensqualität spricht für das Verfahren der SALSA. 


\section{Zusammenfassung}

Die Sinusliftoperation ist ein Verfahren zur Augmentation des atrophierten posterioren Oberkiefers. Im Rahmen dieser Nachuntersuchung wurden die Langzeitergebnisse der minimalinvasiven Subantroskopischen Laterobasalen Sinusbodenaugmentation (SALSA) bewertet.

Im Zeitraum von 1996 bis 2007 wurde in der Mund-, Kiefer- und Gesichtschirurgie der Universitätsklinik Göttingen bei insgesamt 94 Patienten, davon 37 Männer und 57 Frauen, eine Sinuslift-Operation mittels SALSA-Technik durchgeführt.

Bei 39 Patienten mussten beide Kieferhöhlenböden augmentiert werden, bei 55 Patienten erfolgte eine einseitige Sinusbodenelevation. Von 320 Implantaten wurden 295 simultan zur Sinusliftoperation implantiert, 25 Implantate in einem zweizeitigen Verfahren.

Als Implantatsysteme fanden Xive $(n=118)$, Semados $(n=116)$, Frialit-2 $(n=36)$ und ITI $(n=50)$ Anwendung.

Der Beobachtungszeitraum betrug durchschnittlich 78 Monate (Min. 3 Monate, Max. 166 Monate).

Radiologisch wurden die präoperative Knochenhöhe, die vertikale Augmentathöhe sowie der marginale Knochenabbau metrisch im Orthopantomogramm ermittelt. Zur Evaluation des periimplantären Zustandes wurden die Taschentiefen und die Mobilität der Implantate untersucht sowie die Patientenzufriedenheit mit Hilfe eines Fragebogens dokumentiert.

Es wurde eine kumulative Implantatüberlebensrate von 95,8\% im augmentierten Oberkiefer beobachtet. Die Erfolgsrate betrug 94,7\%.

Die Überlebensrate getrennt nach Implantatsystemen ergab für die Frialit-2-Implantate $(n=36)$ $100 \%$, für die Semados-Implantate $(n=116) 98,3 \%$, bei den Xive-Implantaten $(n=118) 95,7 \%$ und bei den ITI-Implantaten ( $\mathrm{n}=50) 88,8 \%$.

Hinsichtlich der Implantatposition im Kiefer betrug die Überlebensrate in der Region des ersten Prämolaren ( $n=94$ ) 96,9\%, beim zweiten Prämolaren ( $n=107)$ 97,2\%, beim ersten Molaren ( $\mathrm{n}=90$ ) 92,6\% und für die Region des zweiten Molaren ( $\mathrm{n}=29$ ) 96,4\%.

Die Überlebensrate der einzeitig inserierten Implantate betrug 96,2\%, beim zweizeitigen Verfahren wurden $92,0 \%$ ermittelt. In Bezug auf die Ausgangsknochenhöhen konnte bei geringen präoperativen Knochenhöhen eine schlechtere Überlebensrate $(93,6 \%)$ gegenüber höheren Ausgangsknochenhöhen (98,2\%) festgestellt werden.

Bei der Messung des Knochenverlustes ergab sich bei unserer Patientengruppe ein mittlerer marginaler Knochenabbau von 2,9 mm. Die Messung der Sondierungstiefen ergab die mittleren Werte von 2,9 $\mathrm{mm}$ mesial und 3,3 $\mathrm{mm}$ distal.

Der Periotestwert betrug durchschnittlich $-2,42$ und bestätigte damit die Osseointegration der Implantate. 
65\% der Patienten bewerteten das Gesamtresultat der Behandlung mit „sehr gut“ und über 90\% der Patienten würden die Behandlung weiter empfehlen. Wichtige Kriterien wie Halt und Kaufunktion des implantatgetragenen Zahnersatzes wurden überwiegend mit „gut“ oder „sehr gut" bewertet.

Abschließend kann zusammengefasst werden, dass die minimalinvasive SALSA-Technik zur Vorbereitung der Implantataufnahme eine umsetzbare, voraussagbare und langfristige Therapiemöglichkeit darstellt, auch bei geringen Ausgangsknochenhöhen. 


\section{Anhang}

\subsection{Abkürzungsverzeichnis}

Abb: Abbildung

ATH: Augmentationshöhe

ATM: Augmentationsmaterial

ß-TCP: $\beta$ - Trikalziumphosphat

C: Caninus

CM: Mittelgesichtspfeiler

Fa: Firma

HA: Hydroxylapatit

HKW: Hinterwand der Kieferhöhle

KEM: Knochenersatzmaterial

m: männlich

M: Molar

Max: Maximum

Min: Minimum

mm: Millimeter

$\mathrm{n}$ : Anzahl

NB: Nasenboden

OPG: Orthopantomogramm

PM: Prämolar

S.A.: Standardabweichung

SALSA: Subantroskopische laterobasale Sinusbodenaugmentation

SAR: Subantralraum

SFT: Sinuslift- Fenstertechnik

SLO: Sinusliftoperation

SM: Sinus maxillaris

SMP: Sinus-Mukoperiost

w: weiblich 


\subsection{Verzeichnis der Abbildungen, Tabellen und Formeln}

\begin{tabular}{|c|c|}
\hline g 1 & ion. \\
\hline Abbildung 2 & gmentation für zweizeitige Implantation... \\
\hline Abbildung 3 & blie. \\
\hline Abbildung 4 & kalibrierte Parodontalsonde \\
\hline Abbildung 5 & kumulative $\mathrm{Ka}$ \\
\hline Abbildung 6 & Kaplan-Meier-Schätzer nach Implantatsystem... \\
\hline Abbildung 7 & Kaplan-Meier-Schätzer nach Position..... \\
\hline Abbildung 8 & Kaplan-Meier-Schätzer nach Augmentationsmaterial.. \\
\hline Abbildung 9 & Kaplan-Meier-Schätzer nach einzeitig/zweizeitig....... \\
\hline Abbildung 10 & Kaplan-Meier-Schätzer nach präoperativer Knochenhöhe.. \\
\hline Abbild & 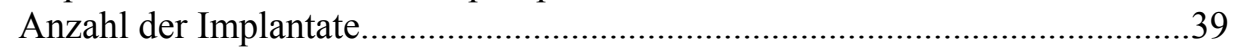 \\
\hline Abbil & Anzahl der Implantate nach einzeitigem/zweizeitigem Verfahren.... \\
\hline hg 13 & Implantatverluste... \\
\hline $\lg 14$ & Kontingenzintervall \\
\hline g 15 & thöhe \\
\hline g 16 & stiefe. \\
\hline 17 & Kon \\
\hline 18 & Wahrscheinlichkeit für Sondierungsbluten in Abhängigkeit des Alters... \\
\hline 19 & ichkeit für Sondierungsbluten in Abhängigkeit der Anzahl \\
\hline Abbi & Kor \\
\hline Abbi & Kol \\
\hline ng 22 & ung insgesamt.... \\
\hline Abbildung 23 & zum Halt des Zahnersatzes..... \\
\hline Abbildung 24 & lung der Kaufunktion..... \\
\hline Abbildung 25 & Anzahl zusätzlicher Zahnarztbesuche bei Problemen mit Zahnersatz........ \\
\hline Abbildung 26 & 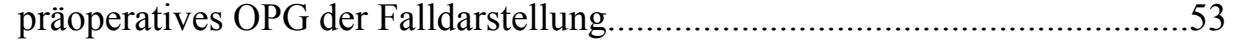 \\
\hline Abbildung 27 & ves OPG............. \\
\hline Abbildung 28 & OPG vom Tag der Nachunt \\
\hline Abbildung 29 & intraorales Foto von vestibulär................. \\
\hline Abbildung 30 & ntraorales Foto von okklusal.... \\
\hline
\end{tabular}

Formel 1 Ermittlung der OPG-bezogenen Zahnbreiten

Tabelle 1

Tabelle 2

Tabelle 3

Tabelle 4

Tabelle 5

Tabelle 6

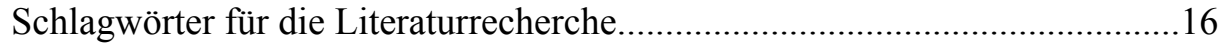

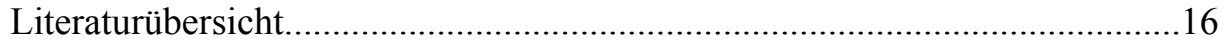

Zahn-Durchschnittsbreiten nach Berkovitz et al. (1992).................................27

Zahnregion-bezogener Faktor a der Durchschnittszahnbreiten.......................27

Beziehung zwischen Periotest und klinischem Lockerungsgrad.........................31

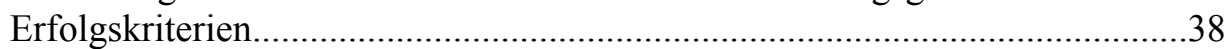




\subsection{Literaturverzeichnis}

Adell R, Lekholm U, Rockler B, Brånemark PI (1981): A 15-year study of osseointegrated implants in the treatment of the edentulous jaw. Int J Oral Surg 10, 387-416

Adell R, Eriksson B, Lekholm U, Brånemark PI, Jemt T (1990): Long-term follow-up study of osseo-integrated implants in the treatment of totally edentulous jaws. Int J Oral Maxillofac Implants $\underline{5}, 347-59$

Albrektsson T, Zarb G, Worthington P, Eriksson AR (1986): The long-term efficacy of currently used dental implants: A review and proposed criteria of success. Int J Oral Maxillofac Implants $\underline{1}, 11-25$

Allen PF, McMillan AS, Walshaw D (2001): A patient-based assessment of implant-stabilized and conventional complete dentures. J Prosthet Dent $\underline{85}$, 141-47

Araújo MG, Lindhe J (2005): Dimensional ridge alterations following tooth extraction. An experimental study in the dog. J Clin Periodontol $\underline{32}$, 212-18

Ardekian L, Oved-Peleg E, Mactei EE, Peled M (2006): The clinical significance of sinus membrane perforation during augmentation of the maxillary sinus. J Oral Maxillofac Surg 64, 277-82

Ariji Y, Kuroki T, Moriguchi S, Ariji E, Kanda S (1994): Age changes in the volume of the human maxillary sinus: a study using computed tomography. Dentomaxillofac Radiol $\underline{23}, 163-$ 8

Arrington ED, Smith WJ, Chambers HG, Bucknell AL, Davino NA (1996): Complications of iliac crest bone graft harvesting. Clin Orthop Relat Res $\underline{329}$, 300-9

Atwood DA (1971): Reduction of residual ridges: a major oral disease entity. J Prosthet Dent $\underline{26}, 266-79$

Atwood DA, Coy WA (1971): Clinical, cephalometric, and densitometric study of reduction of residual ridges. J Prosthet Dent 26, 280-95

Awad MA, Feine JS (1998): Measuring patient satisfaction with mandibular prostheses. Community Dent Oral Epidemiol 26, 400-5

Awad MA, Locker D, Korner-Bitensky N, Feine JS (2000): Measuring the effect of intra-oral implant rehabilitation on health-related quality of life in a randomized controlled clinical trial. $\mathrm{J}$ Dent Res $\underline{79}, 1659-63$

Bain CA, Moy PK (1993): The association between the failure of dental implants and cigarette smoking. Int J Oral Maxillofac Implants $\underline{\text { 8, 609-15 }}$

Balogh K, Molnar L, Schranz D, Huszar G: Gerostomatologie. Barth Verlag, Leipzig 1962

Barone A, Santini S, Sbordone L, Crespi R, Covani U (2006): A clinical study of the outcomes and complications associated with maxillary sinus augmentation. Int $\mathrm{J}$ Oral Maxillofac Implants $\underline{21}, 81-5$ 
Becker ST, Terheyden H, Steinriede A, Behrens E, Springer I, Wiltfang J (2008): Prospective observation of 41 perforations of the Schneiderian membrane during sinus floor elevation. Clin Oral Implants Res $\underline{19}, 1285-89$

Behneke A, Behneke N: Recall und Nachsorge. In: Koeck B, Wagner W (Hrsg.): Praxis der Zahnheilkunde - Implantologie. Urban \& Fischer, München 2004, 265-90

Behneke A, Behneke N, Wagner W (1992): Klinische Ergebnisse mit transgingival inserierten enossalen Implantaten (Bonefit-System). Z Zahnärztl Implantol $\underline{8}$, 97-102

Behneke A, Behneke N, d'Hoedt B, Wagner W (1997): Hard and soft tissue reactions to ITI screw implants: 3-year longitudinal results of a prospective study. Int J Oral Maxillofac Implants $\underline{12}, 749-57$

Berkovitz BKB, Holland GR, Moxham BJ: Color Atlas and Textbook of Oral Anatomy: Histology and Embryology. Mosby-Year Book, St. Louis 1992

Bert M, Missika P, Giovannoli JL: Ätiologie der biologischen Komplikationen. In: Bert M, Missika P, Giovannoli JL (Hrsg): Management der Implantat- Komplikationen. Quintessenz, Berlin 2005, 227-37

Block MS, Kent JN (1993): Maxillary sinus grafting for totally and partially edentulous patients. J Am Dent Assoc 124, 139-43

Block MS, Kent JN (1997): Sinus augmentation for dental implants: the use of autogenous bone. J Oral Maxillofac Surg $\underline{55}$, 1281-86

Blomqvist JE, Alberius P, Isaksson S (1996): Retrospective analysis of one-stage maxillary sinus augmentation with endosseous implants. Int J Oral Maxillofac Implants $\underline{11}, 512-21$

Blomqvist JE, Alberius P, Isaksson S (1997): Sinus inlay bone augmentation: comparison of implant positioning after one- or two- staged procedures. J Oral Maxillofac Surg $\underline{55}$, 804-10

Blomqvist JE, Alberius P, Isaksson S, Linde A, Obrant K (1998): Importance of bone graft quality for implant integration after maxillary sinus reconstruction. Oral Surg Oral Med Oral Pathol Oral Radiol Endod 6ㅜ, 268-74

Bodner L, Kaffe I, Cohen Z, Dayan D (1993): Long-term effect of desalivation on extraction wound healing: a densitrometric study in rats. Dentomaxillofac Radiol 22, 195-98

Bornstein MM, Chappuis V, von Arx T, Buser D (2008): Performance of dental implants after staged sinus floor elevation procedures: 5-year results of a prospective study in partially edentulous patients. Clin Oral Implants Res $\underline{19}, 1034-43$

Boyne PJ, James RA (1980): Grafting of the maxillary sinus floor with autogenous marrow and bone. J Oral Surg $\underline{38}, 613-16$

Brägger U (1994): Radiographic parameters for the evaluation of peri-implant tissues. Periodontol $2000 \underline{4}, 87-97$

Brägger U, Hugel-Pisoni C, Bürgin W, Buser D, Lang NP (1996): Correlations between radiographic, clinical and mobility parameters after loading of oral implants with fixed partial dentures. A 2-year longitudinal study. Clin Oral Implants Res $\underline{7}, 230-9$ 
Brägger U, Gerber C, Joss A, Haenni S, Meier A, Hashorva E, Lang NP (2004): Patterns of tissue remodeling after placement of ITI dental implants using an osteotome technique: a longitudinal radiographic case cohort study. Clin Oral Implants Res $\underline{15}, 158-66$

Brånemark PI, Adell R, Breine U, Hansson BO, Lindström J, Ohlsson A (1969): Intra-osseous anchorage of dental prostheses. I. Experimental studies. Scand J Plast Reconstr Surg $\underline{3}, 81-100$

Brånemark PI: Einführung in die Osseointegration. In: Brånemark PI, Zarb GA, Albrektsson T. (Hrsg.): Gewebeintegrierter Zahnersatz. Osseointegration in klinischer Zahnheilkunde. Quintessenz, Berlin 1985, 11-76

Buch RS, Weibrich G, Wegener J, Wagner J (2002): Patient satisfaction with dental implants. Mund Kiefer Gesichtschir $\underline{6}, 433-36$

Buch RS, Weibrich G, Wagner W (2003): Criteria of success in implantology. Mund Kiefer Gesichtschir $\underline{7,} 42-46$

Buser D, Weber HP, Lang NP (1990): Tissue integration of non-submerged implants. 1-year results of a prospective study with 100 ITI hollow-cylinder and hollow-screw implants. Clin Oral Implants Res $\underline{1}, 33-40$

Buser D, Hoffmann B, Bernard JP, Lussi A, Mettler D, Schenk RK (1998): Evaluation of filling materials in membrane-protected bone defects. A comparative histomorphometric study in the mandible of miniature pigs. Clin Oral Implants Res 9, 137-50

Buser D, Ingimarsson S, Dula K, Lussi A, Hirt HP, Belser UC (2002): Long-term stability of osseointegrated implants in augmented bone: a 5-year prospective study in partially edentulous patients. Int J Periodontics Restorative Dent 22, 109-17

Carlsson GE, Persson G (1967): Morphologic changes of the mandible after extraction and wearing of dentures. Odontol Rev $\underline{18}, 27-54$

Cawood JI, Howell RA (1991): Reconstructive preprosthetic surgery. I. Anatomical considerations. Int J Oral Maxillofac Surg $\underline{20}$, 75-82

Chanavaz M (1990): Maxillary sinus: anatomy, physiology, surgery, and bone grafting related to implantology - eleven years of surgical experience (1979-1990). J Oral Implantol 16, 199209

Chiapasco M, Zaniboni M, Rimondini L (2008): Dental implants placed in grafted maxillary sinuses: a retrospective analysis of clinical outcome according to the initial clinical situation and a proposal of defect classification. Clin Oral Implants Res $\underline{19}$, 416-28

Chuang SK, Wei LJ, Douglass CW, Dodson TB (2002): Risk factors for dental implant failure: a strategy for the analysis of clustered failure-time observations. J Dent Res $\underline{81}, 572-77$

Cibirka RM, Razzoog M, Lang BR (1997): Critical evaluation of patient responses to dental implant therapy. J Prosthet Dent $\underline{78}$, 574-81

Cox JF, Pharoah M (1986): An alternative holder for radiographic evaluation of tissueintegrated prostheses. J Prosthet Dent $\underline{56}, 338-41$

Cox JF, Zarb GA (1987): The longitudinal clinical efficacy of osseointegrated dental implants: a 3 - year report. Int J Oral Maxillofac Implants $\underline{2}$, 91-100 
Davarpanah M, Martinez H, Etienne D, Zabalegui I, Mattout P, Chiche F, Michel JF (2002): A prospective multicenter evaluation of 1,583 3i Implants: 1- to 5-year data. Int J Oral Maxillofac Implants $\underline{17}, 820-28$

Del Fabbro M, Testori T, Francetti L, Weinstein R (2004): Systematic review of survival rates for implants placed in the grafted maxillary sinus. Int J Periodontics Restorative Dent 24, 56577

d'Hoedt B, Schramm-Scherer B (1988): Der Periotestwert bei enossalen Implantaten. Z Zahnärztl Implantol IV, $89-95$

d'Hoedt B, Lukas D, Mühlbradt L, Scholz F, Schulte W, Quante F, Topkaya A (1985): Periotest methods- development and clinical trial. Dtsch Zahnarztl Z $\underline{40}, 113-25$

Drettner B (1980): Pathophysiology of paranasal sinuses with clinical implications. Clin Otolaryngol Allied Sci $\underline{5}, 277-84$

Eckert SE, Meraw SJ, Weaver AL, Lohse CM (2001): Early experience with wide-platform Mk II implants. Part I: Implant survival. Part II: Evaluation of risk factors involving implant survival. Int J Oral Maxillofac Implants $\underline{16}$, 208-16

Eickholz P, Hausmann E (2000): Accuracy of radiographic assessment of interproximal bone loss in intrabony defects using linear measurements. Eur J Oral Sci 108, 70-3

Engelke W, Deckwer I (1997): Endoscopically controlled sinus floor augmentation. A preliminary report. Clin Oral Implants Res $\underline{8}, 527-31$

Engelke W, Schwarzwäller W, Behnsen A, Jacobs HG (2003): Subantroscopic laterobasal sinus floor augmentation (SALSA): an up- to- 5-year clinical study. Int J Oral Maxillofac Implants $\underline{18}, 135-43$

Esposito M, Hirsch JM, Lekholm U, Thomsen P (1998): Biological factors contributing to failures of osseointegrated oral implants. (II). Etiopathogenesis. Eur J Oral Sci 106, 721-64

Esposito M, Grusovin MG, Coulthard P, Worthington HV (2006): The efficacy of various bone augmentation procedures for dental implants: a Cochrane systematic review of randomized controlled clinical trials. Int J Oral Maxillofac Implants 21, 696-710

Ewers R (2005): Maxilla sinus grafting with marine algae derived bone forming material: a clinical report of long-term results. J Oral Maxillofac Surg $\underline{63}$, 1712-23

Fallschüssel GK (1986): Geistig-seelische Einflüsse bei Störungen im Kausystem: FaktenEmpirie- Hypothesen. Dtsch Zahnarztl Z $\underline{41}$, 271-75

Feine JS, de Grandmont P, Boudrias P, Brien N, LaMarche C, Taché R, Lund JP (1994): Within-subject comparisons of implant-supported mandibular prostheses: choice of prosthesis. J Dent Res $\underline{73}, 1105-11$

Ferrigno N, Laureti M, Fanali S (2006): Dental implants placement in conjunction with osteotome sinus floor elevation: a 12-year life-table analysis from a prospective study on 588 ITI implants. Clin Oral Implants Res 17, 194-205

Fredholm U, Bolin A, Andersson L (1993): Preimplant radiographic assessment of available maxillary bone support. Comparison of tomographic and panoramic technique. Swed Dent J 17, 103-09 
Friberg B, Jemt T, Lekholm U (1991): Early failures in 4,641 consecutively placed Brånemark dental implants: a study from stage 1 surgery to the connection of completed prostheses. Int $\mathrm{J}$ Oral Maxillofac Implants $\underline{6}, 142-46$

Friberg B, Dahlin C, Widmark G, Ostman PO, Billström C (2005): One-year results of a prospective multicenter study on Brånemark System implants with a TiUnite surface. Clin Implant Dent Relat Res $\underline{7}, 70-75$

Fugazzotto PA (1999): Sinus floor augmentation at the time of maxillary molar extraction: technique and report of preliminary results. Int J Oral Maxillofac Implants $\underline{14}$, 536-42

Fugazzotto PA, Vlassis J (2003): A simplified classification and repair system for sinus membrane perforations. J Periodontol $\underline{74}, 1534-41$

Fugazzotto PA, Gulbransen HJ, Wheeler SL, Lindsay JA (1993): The use of IMZ osseointegrated implants in partially and completely edentulous patients: success and failure rates of 2,023 implant cylinders up to $60+$ months in function. Int J Oral Maxillofac Implants $\underline{8}$, $617-21$

Garg AK (1999): Augmentation grafting of the maxillary sinus for placement of dental implants: anatomy, physiology, and procedures. Implant Dent $\underline{8}, 36-46$

Gentile MA, Chuang SK, Dodson TB (2005): Survival estimates and risk factors for failure with 6 x 5.7-mm implants. Int J Oral Maxillofac Implants 20, 930-37

Gómez-Román G, Axmann-Krcmar D, d'Hoedt B, Schulte W (1995): Eine Methode zur quantitativen Erfassung und statistischen Auswertung des periimplantären Knochenabbaus. J Stomatol $\underline{92}, 463-71$

Gómez-Román G, Schröer A, Schäfer I, Möws K, Hilliges A (1999): Die Vermessung periimplantärer Knochendefekte auf Röntgenaufnahmen mit Hilfe der digitalen Bildbearbeitung. Eine Reliabilitätsstudie. Z Zahnärztl Implantol 15, 133-8

Goulet JA, Senunas LE, DeSilva GL, Greenfield ML (1997): Autogenous iliac crest bone graft. Complications and functional assessment. Clin Orthop Relat Res $\underline{339}, 76-81$

Haas R, Mensdorff-Pouilly N, Mailath G, Watzek G (1996): Survival of 1,920 IMZ implants followed for up to 100 months. Int J Oral Maxillofac Implants 11, 581-88

Hallman M, Hedin M, Sennerby L, Lundgren S (2002): A prospective 1-year clinical and radiographic study of implants placed after maxillary sinus floor augmentation with bovine hydroxyapatite and autogenous bone. J Oral Maxillofac Surg $\underline{60}$, 277-84

Hanisch O, Cortella CA, Boskovic MM, James RA, Slots J, Wikesjö UM (1997): Experimental peri-implant tissue breakdown around hydroxyapatite-coated implants. J Periodontol $\underline{68}, 59-66$

Haßfeld S, Streib S, Sahl H, Stratmann U, Fehrentz D, Zöller J (1998): Low-doseComputertomographie des Kieferknochens in der präimplantologischen Diagnostik. Mund Kiefer Gesichtschir 2, 188-93

Heary RF, Schlenk RP, Sacchieri TA, Barone D, Brotea C (2002): Persistent iliac crest donor site pain: independent outcome assessment. Neurosurgery $\underline{50}, 510-16$

Heitz-Mayfield LJ (2008): Peri-implant diseases: diagnosis and risk indicators. J Clin Periodontol $\underline{35}, 292-304$ 
Herberhold C (1982): Physiologie und Pathophysiologie der Nasennebenhöhlen. Arch Otorhino-laryngol 235, 1-40

Heydecke G, Locker D, Awad MA, Lund JP, Feine JS (2003): Oral and general health-related quality of life with conventional and implant dentures. Community Dent Oral Epidemiol 31, $161-68$

Higuchi KW, Block MS (1993): Current trends in implant reconstruction. Int J Oral Maxillofac Surg $\underline{51}, 7-19$

Hirsch JM, Ericsson I (1991): Maxillary sinus augmentation using mandibular bone grafts and simultaneous installation of implants. A surgical technique. Clin Oral Implants Res 2, 91-96

Hönig JF, Merten HA, Heinemann DE (1999): Risk of transmission of agents associated with Creutzfeldt-Jakob disease and bovine spongiform encephalopathy. Plast Reconstr Surg 103, 1324-25

Homann C, Fillies T, Joos U, Kleinheinz J (2004): Bewertung implantologischer Versorgungen aus Patientensicht - Literaturübersicht. Z Zahnärztl Impl 20, 168 - 85

Horch HH, Sader R, Pautke C, Neff A, Deppe H, Kolk A (2006): Synthetic, pure-phase betatricalcium phosphate ceramic granules (Cerasorb) for bone regeneration in the reconstructive surgery of the jaws. Int J Oral Maxillofac Surg $\underline{35}, 708-13$

Huang YF, Zhou L, Song GB (2004): Clinical application of maxillary sinus augmentation, bone graft and simultaneously placement of implant with trephine bur. Zhonghua Kou Qiang Yi Xue Za Zhi 39, 153-4

Hürzeler MB: Versorgung der augmentierten Kieferhöhle mit implantatgetragenem Zahnersatz. Eine tierexperimentelle und klinische Studie. Quintessenz, Berlin 1997

Hürzeler MB, Kirsch A, Ackermann KL, Quinones CR (1996): Reconstruction of the severely resorbed maxilla with dental implants in the augmented maxillary sinus: a 5-year clinical investigation. Int J Oral Maxillofac Implants $\underline{11}$, 466-75

Humphries S, Devlin H, Worthington H (1989): A radiographic investigation into bone resorption of mandibular alveolar bone in elderly edentulous adults. J Dent 17, 94-96

Iglhaut GM (2001): Der Einsatz modifizierter Osteotome zur Ausfertigung des Implantatlagers. Dent Implantol $\underline{5}, 14-27$

Iizuka T, Miller SC, Marks SC Jr. (1992): Alveolar bone remodeling after tooth extraction in normal and osteopetrotic (ia) rats. J Oral Pathol Med 21, 150-55

Isaksson S (1994): Evaluation of three bone grafting techniques for severly resorbed maxillae in conjunction with immediate endosseous implants. Int J Oral Maxillofac Implants $\underline{9}, 679-88$

Ivanoff CJ, Gröndahl K, Sennerby L, Bergström C, Lekholm U (1999): Influence of variations in implant diameters: a 3- to 5-year retrospective clinical report. Int J Oral Maxillofac Implants $\underline{14}, 173-80$

Jaffin RA, Berman CL (1991): The excessive loss of Branemark fixtures in type IV bone: a 5year analysis. J Periodontol $\underline{62}, 2-4$ 
Jahn M, d'Hoedt B (1992): Zur Definition des Erfolges bei dentalen Implantaten. Ein Vergleich verschiedener Kriterien. Z Zahnärztl Implantol $\underline{8}$, 221-26

Jansen VK, Augthun M, Richter EJ, Spiekermann H (1993): Zur Genauigkeit des Orthopantomogramms bei der Bestimmung des Knochenabbaus an IMZ-Implantaten. Z Zahnärztl Implantol $\underline{9}, 200-04$

Jensen OT: Guided bone graft augmentation. In: Buser D, Dahlin C, Schenk RK (Hrsg.): Guided bone regeneration in implant dentistry. Quintessence, Chicago 1994, 235-64

Jensen SS, Terheyden H (2009): Bone augmentation procedures in localized defects in the alveolar ridge: clinical results with different bone grafts and bone-substitute materials. Int $\mathrm{J}$ Oral Maxillofac Implants 24, 218-36

Jensen OT, Shulman LB, Block MS, Iacono VJ (1998): Report of the Sinus Consensus Conference of 1996. Int J Oral Maxillofac Implants $\underline{13}$, 11- 45

Johansson LA, Isaksson S, Lindh C, Becktor JP, Sennerby L (2010): Maxillary sinus floor augmentation and simultaneous implant placement using locally harvested autogenous bone chips and bone debris: A prospective clinical study. Int J Oral Maxillofac Surg 68, 837-44

Jovanovic SA, Spiekermann H, Richter EJ (1992): Bone regeneration around titanium dental implants in dehisced defect sites: a clinical study. Int J Oral Maxillofac Implants $\underline{7}, 233-45$

Kahnberg KE, Ekestubbe A, Gröndahl K, Nilsson P, Hirsch JM (2001): Sinus lifting procedure. I. One-stage surgery with bone transplant and implants. Clin Oral Implants Res $\underline{12}, 479-87$

Kahnberg KE, Vannas-Löfqvist L (2008): Sinus lift procedure using a 2-stage surgical technique: I. Clinical and radiographic report up to 5 years. Int J Oral Maxillofac Implants $\underline{23}$, 876-84

Kaptein ML, Hoogstraten J, de Putter C, de Lange GL, Blijdorp PA (1998): Dental implants in the atrophic maxilla: measurements of patients' satisfaction and treatment experience. Clin Oral Implants Res $\underline{9}, 321-26$

Keller EE, Eckert SE, Tolman DE (1994): Maxillary antral and nasal one-stage inlay composite bone graft: preliminary report on 30 recipient sites. J Oral Maxillofac Surg 52, 438-47

Keller U (1995): Die Darstellung des periimplantären Knochenabbaus im Röntgenbild. Z Zahnärztl Implantol 11, 88-91

Kent JN, Block MS (1989): Simultaneous maxillary sinus floor bone grafting and placement of hydroxylapatite-coated implants. J Oral Maxillofac Surg 47, 238- 42

Khoury F, Pingel D, Joos U (1993): Die Sinusbodenelevation mit simultaner Implantation unter Verwendung von Knochentransplantaten aus dem Unterkiefer. Z Zahnärztl Implantol 9, 175-80

Kirsch A, Ackermann KL, Hürzeler MB, Hutmacher D: Sinus grafting with porous hydroxyapatite. In: Jensen OT (Hrsg.): The sinus bone graft. Quintessence, Chicago 1999, 79-94

Kitamura E, Stegaroiu R, Nomura S, Miyakawa O (2004): Biomechanical aspects of marginal bone resorption around osseointegrated implants: considerations based on a three-dimensional finite element analysis. Clin Oral Implants Res $\underline{15}$, 401-12 
Knöfler W, Knöfler A, Graf HL (2004): Die Überlebenswahrscheinlichkeit von Implantaten in einer zahnärztlichen Praxis im Zeitraum von zehn Jahren. Z Zahnärtzl Implantol 20, 230-46

Kovács A (1998): Horizontaler und periimplantärer Knochenabbau in freien und gefäßgestielten Beckenkammtransplantaten. Z Zahnärztl Implantol 14, 143-50

Kreisler M, Moritz O, Weihe Ch, D'Hoedt B (2006): Die externe Sinusbodenelevation vor dem Hintergrund der Evidenzbasierten Medizin. Teil 1: Allgemeine Aspekte und Einfluss des Augmentationsmaterials auf die Implantatprognose. Z Zahnärztl Impl 22, 299-323

Kübler NR, Will C, Depprich R, Betz T, Reinhart E, Bill JS, Reuther JF (1999): Vergleichende Untersuchungen zur Sinusbodenelevation mit autogenem oder allogenem Knochengewebe. Mund Kiefer Gesichtschir $\underline{3}, 53-60$

Lane JM, Tomin E, Bostrom MP (1999): Biosynthetic bone grafting. Clin Orthop Relat Res $\underline{367}, 107-17$

Langer B, Langer L: Use of allografts for sinus grafting. In: Jensen OT (Hrsg.): The sinus bone graft. Quintessence, Chicago 1999, 69-78

Larheim TA, Eggen S (1982): Measurements of alveolar bone height at tooth and implant abutments on intraoral radiographs. A comparison of reproducibility of Eggen technique utilized with and without a bite impression. J Clin Periodontol $\underline{9}, 184-92$

Lekholm U (1986): Osseointegrated implants in clinical practice. J Oral Implantol 12, 357-64

Lekholm U, Zarb GA: Patient selection and preparation. In: Brånemark PI, Zarb GA, Albrektsson T (Hrsg.): Tissue integrated prostheses: Osseointegration in clinical dentistry. Quintessence, Chicago 1985, 199-209

Lekholm U, Wannfors S, Isaksson S, Adielsson B (1999): Oral implants in combination with bone grafts. A 3-year retrospective multicenter study using the Brånemark implant system. Int J Oral Maxillofac Surg $\underline{28}, 181-87$

Lemmerman KJ, Lemmerman NE (2005): Osseointegrated dental implants in private practice: a long-term case series study. J Periodontol $\underline{76}, 310-19$

Lindh T, Gunne J, Tillberg A, Molin M (1998): A meta-analysis of implants in partial edentulism. Clin Oral Implants Res $\underline{9}$ 80-90

Lindhe J, Meyle J (2008): Peri-implant diseases: Consensus report of the sixth european workshop on periodontology. J Clin Periodontol $\underline{35}, 282-5$

Lindhe J, Berglundh T, Ericsson I, Liljenberg B, Marinello C (1992): Experimental breakdown of peri-implant and periodontal tissues. A study in the beagle dog. Clin Oral Implants Res $\underline{3}, 9-$ 16

Lindquist LW, Carlsson GE, Jemt T (1996): A prospective 15-year follow-up study of mandibular fixed prostheses supported by osseointegrated implants. Clinical results and marginal bone loss. Clin Oral Implants Res $\underline{7}, 329-36$

Lundgren S, Moy P, Johansson C, Nilsson H (1996): Augmentation of the maxillary sinus floor with particulated mandible: a histologic and histomorphometric study. Int J Oral Maxillofac Implants $\underline{11}, 760-6$ 
Mardinger O, Nissan J, Chaushu G (2007): Sinus floor augmentation with simultaneous implant placement in the severely atrophic maxilla: technical problems and complications. J Periodontol $\underline{78}, 1872-77$

Martini M: Sinuslift und endossale Implantation. Eine retrospective Sechsjahresstudie. Med. Diss., Bonn 2001

Mazor Z, Peleg M, Garg AK, Luboshitz J (2004): Platelet-rich plasma for bone graft enhancement in sinus floor augmentation with simultaneous implant placement: patient series study. Implant Dent $\underline{13}, 65-72$

McAllister BS, Haghighat K (2007): Bone augmentation techniques. J Periodontol $\underline{78}$, 377-96

McDermott NE, Chuang SK, Woo VV, Dodson TB (2006): Maxillary sinus augmentation as a risk factor for implant failure. Int J Oral Maxillofac Implants 211, 366-74

Meijer HJ, Raghoebar GM, Van't Hof MA, Geertman ME, Van Oort RP (1999): Implantretained mandibular overdentures compared with complete dentures; a 5-years' follow-up study of clinical aspects and patient satisfaction. Clin Oral Implants Res 10, 238-44

Mercier P (1985): Ridge form in preprosthetic surgery. Oral Surg Oral Med Oral Pathol $\underline{60}$, $235-43$

Merkx MA, Maltha JC, Stoelinga PJ (2003): Assessment of the value of anorganic bone additives in sinus floor augmentation: a review of clinical reports. Int J Oral Maxillofac Surg $\underline{32}, 1-6$

Meyer C, Chatelain B, Benarroch M, Garnier JF, Ricbourg B, Camponovo T (2009): Massive sinus-lift procedures with beta-tricalcium phosphate: long-term results. Rev Stomatol Chir Maxillofac 110, 69-75

Meyle J: Vergleichende Untersuchungen der Tastsensibilität beim natürlichen Zahn und beim Tübinger Implantat. Med. Diss., Tübingen 1984

Misch CE (1987): Maxillary sinus augmentation for endosteal implants: organized alternative treatment plants. Int J Oral Implantol $\underline{4}, 49-58$

Misch, CE: Bone density: A key determinant in treatment planning. In: Misch CE (Hrsg.): Contemporary Implant Dentistry. Mosby, St. Louis 1999, 130-46

Misch CE, Dietsh F (1993): Bone-grafting materials in implant dentistry. Implant Dent 2, 15867

Misch CE, Dietsh F (1994): Endosteal implants and iliac crest grafts to restore severely resorbed totally edentulous maxillae - a retrospective study. J Oral Implantol 20, 100-10

Mombelli A, Lang NP (1994): Clinical parameters for the evaluation of dental implants. Periodontol $2000 \underline{4}, 81-6$

Mombelli A, Lang NP (1998): The diagnosis and treatment of peri-implantitis. Periodontol 2000 17, $63-76$

Moy PK, Lundgren S, Holmes RE (1993): Maxillary sinus augmentation: histomorphometic analysis of graft matrials for maxillary sinus floor augmentation. J Oral Maxillofac Surg 51, $857-62$ 
Moy PK, Medina D, Shetty V, Aghaloo TL (2005): Dental implant failure rates and associated risk factors. Int J Oral Maxillofac Implants $\underline{20}, 569-77$

Mundt T, Mack F, Schwahn C, Biffar R (2006): Private practice results of screw-type tapered implants: survival and evaluation of risk factors. Int J Oral Maxillofac Implants 21, 607-14

Naert I, Quirynen M, Theuniers G, van Steenberghe D (1991): Prosthetic aspects of osseointegrated fixtures supporting overdentures. A 4 - year report. J Prosthet Dent $\underline{65}, 671-80$

Naert I, Quirynen M, van Steenberghe D, Darius P (1992): A six-year prosthodontic study of 509 consecutively inserted implants for the treatment of partial edentulism. J Prosthet Dent 67 , $236-45$

Naert I, Koutsikakis G, Quirynen M, Duyck J, van Steenberghe D, Jacobs R (2002): Biologic outcome of implant-supported restorations in the treatment of partial edentulism. Part I: a longitudinal clinical evaluation. Clin Oral Impl Res $\underline{13}$, 381-89

Neukam FW, Esser E (2000): Implantologie. Mund Kiefer Gesichtschir 4, 249-56

Neukam FW, Schultze-Mosgau S: Implantate bei ausgedehnten Knochendefiziten. In: Koeck W, Wagner W (Hrsg.): Praxis der Zahnheilkunde - Implantologie. Urban \& Fischer, München 2004, 184-228

Neukam FW, Scheller H, Günay H (1989): Experimentelle und klinische Untersuchungen zur Auflagerungsosteoplastik in Kombination mit enossalen Implantaten. Z Zahnärztl Implantol $\underline{5}$, $235-41$

Nevins M, Langer B (1993): The successful application of osseointegrated implants to the posterior jaw: a long-term retrospective study. Int J Oral Maxillofac Implants $\underline{8}, 428-32$

Nevins M, Mellonig JT, Clem DS 3rd, Reiser GM, Buser DA (1998): Implants in regenerated bone: long-term survival. Int J Periodontics Restorative Dent $\underline{18}$, 34-45

Niedhart C, Pingsmann A, Jürgens C, Marr A, Blatt R, Niethard FU (2003): Complications after harvesting of autologous bone from the ventral and dorsal iliac crest- a prospective, controlled study. Z Orthop Ihre Grenzgeb $\underline{141}$, 481-6

Nkenke E, Schlegel A, Schultze-Mosgau S, Neukam FW, Wiltfang J (2002): The endoscopically controlled osteotome sinus floor elevation: a preliminary prospective study. Int J Oral Maxillofac Implants 17, 557- 66

Olson JW, Dent CD, Dominici JT, Lambert PM, Bellome J, Bichara J, Morris HF (1997): The influence of maxillary sinus augmentation on the success of dental implants through secondstage surgery. Implant Dent $\underline{6}, 225-28$

Olson JW, Dent CD, Morris HF, Ochi S (2000): Long-term assessment (5 to 71 months) of endosseous dental implants placed in the augmented maxillary sinus. Ann Periodontol $\underline{5,152-}$ 156

Ozyuvaci H, Aktas I, Yerit K, Aydin K, Firatli E (2005): Radiological evaluation of sinus lift operation: what the general radiologist needs to know. Dentomaxillofac Radiol 34, 199-204

Parikh SN (2002): Bone graft substitutes: past, present, future. J Postgrad Med 48, 142-48 
Peleg M, Mazor Z, Chaushu G, Garg AK (1998): Sinus floor augmentation with simultaneous implant placement in the severely atrophic maxilla. J Periodontol $\underline{69}, 1397-1403$

Peleg M, Chaushu G, Mazor Z, Ardekian L, Bakoon M (1999): Radiological findings of the post-sinus lift maxillary sinus: a computerized tomography follow-up. J Periodont $\underline{70}, 1564-73$

Peleg M, Garg AK, Mazor Z (2006): Predictability of simultaneous implant placement in the severly atrophic posterior maxilla: A 9-year longitudinal experience study of 2132 implants placed into 731 human sinus grafts. Int J Oral Maxillofac Implants 21, 94-102

Perry J, Lenchewski E (2004): Clinical performance and 5-year retrospective evaluation of Frialit-2 implants. Int J Oral Maxillofac Implants 19, 887-91

Pham AN, Fiorellini JP, Paquette D, Williams RC, Weber HP (1994): Longitudinal radiographic study of crestal bone levels adjacent to non-submerged dental implants. J Oral Implantol 20, 26-34

Pikos MA (1999): Block autografts for localized ridge augmentation: Part I. The posterior maxilla. Implant Dent $\underline{8}, 279-85$

Pjetursson BE, Rast C, Brägger U, Schmidlin K, Zwahlen M, Lang NP (2009): Maxillary sinus floor elevation using the (transalveolar) osteotome technique with or without grafting material. Part I: implant suvival and patient's perception. Clin Oral Implants Res 20, 667-76

Pontoriero R, Tonelli MP, Carnevale G, Mombelli A, Nyman SR, Lang NP (1994): Experimentally induced peri-implant mucositis. A clinical study in humans. Clin Oral Implants Res $\underline{5}, 254-59$

Pröbster L, Weber H (1989): Implantatgehaltener Zahnersatz im zahnlosen Unterkiefer in der subjektiven Bewertung des Patienten. Z Zahnärztl Implantol 5 , 194-97

Proussaefs P, Lozada J, Kim J, Rohrer MD (2004): Repair of the perforated sinus membrane with a resorbable collagen membrane: a human study. Int J Oral Maxillofac Implants $\underline{19}, 413-$ 20

Quirynen M, Listgarten MA (1990): Distribution of bacterial morphotypes around natural teeth and titanium implants ad modum Brånemark. Clin Oral Implants Res 1, 8-12

Raghoebar GM, Timmenga NM, Reintsema H, Stegenga B, Vissink A (2001): Maxillary bone grafting for insertion of endosseous implants: results after 12- 124 months. Clin Oral Implants Res $\underline{12}, 279-86$

Reinhardt C, Kreusser B (2000): Retrospektive Studie nach Implantation mit Sinuslift und Cerasorb-Augmentation. Dent Implantol $\underline{4}, 18-26$

Riediger D (1989): Bone grafting by revascularized iliac crest transplants in the face and neck region, Microsurgical tissue transplantation. Quintessence, 59-68

Roos-Jansaker AM, Lindahl C, Renvert H, Renvert S (2006): Nine- to fourteen-year follow-up of implant treatment. Part II: presence of peri-implant lesions. J Clin Periodontol 33, 290-95

Rosen PS, Summers R, Mellado JR, Salkin LM, Shanaman RH, Marks MH, Fugazzotto PA (1999): The bone-added osteotome sinus floor elevation technique: multicenter retrospective report of consecutively treated patients. Int J Oral Maxillofac Implants $\underline{14}, 853-58$ 
Rosenbauer KA, Engelhardt JP, Koch H, Stüttgen U (Hrsg.): Klinische Anatomie der Kopfund Halsregion für Zahnmediziner. Thieme, Stuttgart 1998, 236

Rüdiger S, Flemming F (1995): Periimplantäre Diagnostik. Implantologie 4 , 301-13

Schlegel KA, Karascholi T, Fenner M, Nkenke E (2007): Klinische und radiologische Ergebnisse nach Augmentationen - eine prospektive Evaluation. Mund Kiefer Gesichtschir 11, 209-19

Schliephake H, Neukam FW, Schmelzeisen R, Wichmann M (1999): Long-term results of endosteal implants used for restoration of oral function after oncologic surgery. Int $\mathrm{J}$ Oral Maxillofac Surg 28, 260-65

Schnürer SM, Goppp U, Kühn KD, Breusch SJ (2003): Bone substitutes. Orthopade 르, 2-10

Schramm-Scherer B, Behneke N, Reiber T, Tetsch P (1989): Röntgenologische Untersuchungen zur Belastung von Implantaten im zahnlosen Unterkiefer. Z Zahnärztl Implantol $\underline{5}, 185-92$

Schulte W, d'Hoedt B, Lukas D, Mühlbradt L, Scholz F, Bretschi J, Frey D, Gudat H, König M, Markl M, Quante F, Schief A, Topkaya A (1983): Periotest - neues Messverfahren der Funktion des Parodontiums. Zahnärztl Mitt 11, 1229-40

Schwartz-Arad D, Herzberg R, Dolev E (2004): The prevalence of surgical complications of the sinus graft procedure and their impact on implant survival. J Periodontol 75, 511-16

Scurria MS, Morgan ZV 4th, Guckes AD, Li S, Koch G (1998): Prognostic variables associated with implant failure: a retrospective effectiveness study. Int J Oral Maxillofac Implants $\underline{13}$, 400-06

Shatkin TE, Shatkin S, Oppenheimer BD, Oppenheimer AJ (2007): Mini dental implants for long-term fixed and removable prosthetics: a retrospective analysis of 2514 implants placed over a five-year period. Compend Contin Educ Dent 28, 92-99

Shlomi B, Horowitz I, Kahn A, Dobriyan A, Chaushu G (2004): The effect of sinus membrane perforation and repair with lambone on the outcome of maxillary sinus floor augmentation: a radiographic assessment. Int J Oral Maxillofac Implants $\underline{19}$, 559-62

Simion M, Jovanovic SA, Tinti C, Benfenati SP (2001): Long-term evaluation of osseointegrated implants inserted at the time or after vertical ridge augmentation. A retrospective study on 123 implants with 1-5 year follow-up. Clin Oral Implants Res 12, 35-45

Simion M, Fontana F, Rasperini G, Maiorana C (2004): Long-term evaluation of osseointegrated implants placed in sites augmented with sinus floor elevation associated with vertical ridge augmentation: a retrospective study of 38 consecutive implants with 1- to 7-year followup. Int J Periodontics Restorative Dent 24, 208-21

Smiler DG, Johnson PW, Lozada JL, Misch C, Rosenlicht JL, Tatum OH Jr., Wagner JR (1992): Sinus lift grafts and endosseous implants. Treatment of the atrophic posterior maxilla. Dent Clin North Am $\underline{36}, 151-86$

Snauwaert K, Duyck J, van Steenberghe D, Quirynen M, Naert I (2000): Time dependent failure rate and marginal bone loss of implant supported prostheses: a 15-year follow-up study. Clin Oral Investig $2000 \underline{4}, 13-20$ 
Spiekermann H, Jansen VK, Richter EJ (1995): A 10-year follow-up study of IMZ and TPS implants in the edentulous mandible using bar-retained overdentures. Int J Oral Maxillofac Implants $\underline{10}, 231-43$

St John TA, Vaccaro AR, Sah AP, Schaefer M, Berta SC, Albert T, Hilibrand A (2003): Physical and monetary costs associated with autogenous bone graft harvesting. Am J Orthop $\underline{32}, 18-23$

Stricker A, Voss PJ, Gutwald R, Schramm A, Schmelzeisen R (2003): Maxillary sinus floor augmention with autogenous bone grafts to enable placement of SLA-surfaced implants: preliminary results after 15-40 months. Clin Oral Implants Res 14, 207-12

Strid KG: Radiographic results. In: Brånemark PI, Zarb G, Albrektsson $\mathrm{T}$ (Hrsg.): Gewebeintegrierter Zahnersatz. Osseointegration in klinischer Zahnheilkunde. Quintessence, Berlin 1985, 183-94

Strietzel FP (2004): Sinusbodenelevation und - augmentation: Evidenzgestützte Aussagen zu Prognose und Risikofaktoren. Mund Kiefer Gesichts Chir $\underline{8}$, 93-105

Strietzel FP, Lange KP, Svegar M, Hartmann HJ, Küchler I (2004): Retrospective evaluation of the success of oral rehabilitation using the Frialit-2 implant system. Part 1: Influence of topographic and surgical parameters. Int J Prosthodont 17, 187-94

Summers RB (1994): A new concept in maxillary implant surgery: the osteotome technique. Compendium 15, 152-56

Summers RB (1996): Conservative osteotomy technique with simultaneous implant insertion. Dent Implantol Update $\underline{7}, 49-53$

Summers RB (1998): Sinus floor elevation with osteotomes. J Esthet Dent 10, 164-71

Szabo G, Keck B, d'Hoedt B (1991): Präimplantologische Diagnostik mit individuellen Röntgenschablonen im Orthopantomogramm. Z Zahnärztl Implantol ㄱ, 33-36

Szabo G, Huys L, Coulthard P, Maiorana C, Garagiola U, Barabas J, Nemeth Z, Hrabak K, Suba Z (2005): A prospective multicenter randomized clinical trial of autogenous bone versus beta-tricalcium phosphate graft alone for bilateral sinus elevation: histologic and histomorphometric evaluation. Int J Oral Maxillofac Implants 20, 371-81

Tadjoedin ES, de Lange GL, Holzmann PJ, Kulper L, Burger EH (2000): Histological observations on biopsies harvested following sinus floor elevation using a bioactive glass material of narrow size range. Clin Oral Impl Res $\underline{11}, 334-44$

Tallgren A (1972): The continuing reduction of the residual alveolar ridges in complete denture wearers: a mixed-longitudinal study covering 25 years. J Prosthet Dent $\underline{27}, 120-32$

Tatum H Jr. (1986): Maxillary and sinus implant reconstructions. Dent Clin North Am $\underline{30}$, 20729

Tatum OH Jr., Lebowitz MS, Tatum CA, Borgner RA (1993): Sinus augmentation. Rationale, development, long-term results. N Y State Dent J $\underline{59}, 43-48$

Tawil G, Mawla M (2001): Sinus floor elevation using a bovine bone mineral (Bio-Oss) with or without the concomitant use of a bilayered collagen barrier (Bio-Gide): a clinical report of immediate and delayed implant placement. Int J Oral Maxillofac Implants $\underline{16}$, 713-21 
Tetsch J, Tetsch P, Lysek DA (2010): Long-term results after lateral and osteotome technique sinus floor elevation: a retrospective analysis of 2190 implants over a time period of 15 years. Clin Oral Impl Res 21, 497-503

Timmenga NM, Raghoebar GM, Boering G, van Weissenbruch R (1997): Maxillary Sinus Function after sinus lifts for the insertion of dental implants. J Oral Maxillofac Surg $\underline{55}$, 936-39

Toffler M (2004): Osteotome-mediated sinus floor elevation: A clinical report. Int J Oral Maxillofac Implants $\underline{19}, 266-73$

Triplett RG, Schow SR (1996): Autologous bone grafts and endosseous implants: complementary techniques. J Oral Maxillofac Surg 54, 486-94

Ulm C, Solar P, Blahout R, Matejka M, Gruber H (1992): Reduction of the compact and cancellous bone substances of the edentulous mandible caused by resorption. Oral Surg Oral Med Oral Pathol 74, 131-36

Ulm CW, Solar P, Krennmair G, Matejka M, Watzek G (1995): Incidence and suggested surgical management of septa in sinus-lift procedures. Int J Oral Maxillofac Implants $\underline{10}, 462-$ 65

Ulm C, Kneissel M, Schedle A, Solar P, Matejka M, Schneider B, Donath K (1999): Characteristic features of trabecular bone in edentulous maxillae. Clin Oral Implants Res $\underline{10}$, 459-67

Valentin AH, Barber D, Seckinger RJ, Benner KU (2000): Die Bedeutung osteokonduktiver Eigenschaften von Glaspartikeln uniformer Größe (Biogran) am Beispiel der Sinusbodenelevation - erste Untersuchungsergebnisse. Implantologie $\underline{1}, 35-43$

van den Bergh JP, ten Bruggenkate CM, Krekeler G, Tuinzing DB (1998): Sinusfloorelevation and grafting with autogenous iliac crest bone. Clin Oral Implants Res $\underline{9}, 429-35$

van den Bergh JP, ten Bruggenkate CM, Disch FJ, Tuinzing DB (2000): Anatomical aspects of sinus floor elevations. Clin Oral Implants Res $\underline{11}$, 256-65

Vlassis JM, Fugazzotto PA (1999): A classification system for sinus membrane perforations during augmentation procedures with options for repair. J Periodontol $\underline{70}$, 692-99

Wagner W, Al Nawas B: Implantatmaterialen und Konstruktionsprinzipien der Implanate aus chirurgischer Sicht. In: Koeck B, Wagner W (Hrsg.): Praxis der Zahnheilkunde-Implantologie. Urban \& Fischer, München 2004, 61-97

Wallace SS, Froum SJ (2003): Effect of maxillary sinus augmentation on the survival of endosseous dental implants. A systematic review. Ann Periodontol $\underline{8}, 328-43$

Walton JN, MacEntee MI (1994): Problems with prostheses on implants: a retrospective study. J Prosthet Dent $71,283-88$

Watzek G, Mailath-Pokorny G: Zahnärztliche Implantate. In: Schwenzer N, Ehrenfeld M, (Hrsg.): Zahnärztliche Chirurgie. Thieme, Stuttgart 2000, 127-168

Watzek G, Weber R, Bernhart T, Ulm C, Haas R (1998): Treatment of patients with extreme maxillary atrophy using sinus floor augmentation and implants: preliminary results. Int $\mathrm{J}$ Oral Maxillofac Surg 27, 428-34 
Watzek G, Ulm CW, Haas R: Anatomic and physiologic fundamentals of sinus floor augmentation. In: Jensen OT (Hrsg.): The sinus bone graft. Quintessence, Chicago 1999, 31-47

Weber HP, Crohin CC, Fiorellini JP (2000): A 5-year prospective clinical and radiographic study of non-submerged dental implants. Clin Oral Implants Res $\underline{11}, 144-53$

Weingart D, Schilli W, Strub JR (1992): Präprothetische Chirurgie und Implantologie. Einfluss der oralen Implantologie auf die präprothetische Chirurgie beim zahnlosen Patienten. Schweiz Monatsschr Zahnmed 102, 1074-85

Weingart D, Werkmeister R, Joos U, Röck1 J, Pingel D (1996): Korrektur der sagittalen Diskprepanz bei starker Atrophie des Oberkieferalveolarkammes. Dtsch Zahnärztl Z 51, 46

Wheeler SL, Holmes RE, Calhoun CJ (1996): Six-year clinical and histologic study of sinus-lift grafts. Int J Oral Maxillofac Implants 11, 26-34

Wictorin L (1964): Bone resorption in cases with complete upper denture. A quantitative roentgenographic-photogrammetric study. Acta Radiol Diagn 228, 1-97

Wilkert-Walter C, Jaenicke S, Spuentrup E, Laurin TH (2002): Kieferhöhlenuntersuchung nach Sinusbodenelevation kombiniert mit autologer Onlay-Osteoplastik. Mund Kiefer Gesichts Chirurgie $\underline{6}, 336-40$

Wiltfang J, Schultze-Mosgau S, Nkenke E, Thorwarth M, Neukam FW, Schlegel KA (2005): Onlay augmentation versus sinuslift procedure in the treatment of the severly resorbed maxilla: a 5-year comparative longitudinal study. Int J Oral Maxillofac Surg $\underline{34}, 885-89$

Woo I, Le BT (2004): Maxillary sinus floor elevation: review of anatomy and two techniques. Implant Dent 13, 28-32

Zijderveld SA, Zerbo IR, van den Bergh JP, Schulten EA, ten Bruggenkate CM (2005): Maxillary sinus floor augmentation using a beta-tricalcium phosphate (Cerasorb) alone compared to autogenous bone grafts. Int J Oral Maxillofac Implants $\underline{20}, 432-40$

Zitzmann NU (2004): Die Folgen der Zahnlosigkeit für das Individuum. Dtsch Zahnärztl Z $\underline{59}$, $617-25$

Zitzmann NU, Schärer P (1998): Sinus elevation procedures in the resorbed posterior maxilla: Comparison of the crestal and lateral approaches. Clin Surg Oral Med Oral Pathol Oral Radiol Endod $\underline{85}, 8-17$ 


\section{Danksagung}

Mein besonderer Dank gilt Herrn Prof. Dr. med. Dr. med. dent. Wilfried Engelke für die freundliche Überlassung des Themas dieser Arbeit sowie seine Unterstützung, Betreuung und zahllosen Anregungen während der gesamten Zusammenarbeit.

Auch allen Mitarbeiter(innen) der Poliklinik für Mund-, Kiefer- und Gesichtschirurgie des Klinikums Göttingen, die mich bei der klinischen Nachuntersuchung der Patienten unterstützt haben, möchte ich an dieser Stelle danken.

Weiterhin danke ich Frau Katharina Lange und Herrn Simon Schneider der Medizinischen Statistik Göttingen für ihre Unterstützung bei der Strukturierung und der statistischen Auswertung meiner umfangreichen Datensammlung.

Nicht zuletzt sei den Patienten gedankt, die in dieser Studie erfasst wurden und bereitwillig an der Untersuchung teilnahmen. 